Foundations and Trends ${ }^{\circledR}$ in

Communications and Information Theory

Vol. 8, No. 4 (2011) 259-381

(C) 2012 E. Şaşoğlu

DOI: $10.1561 / 0100000041$

\title{
Polarization and Polar Codes
}

\author{
By Eren Şaşoğlu
}

\section{Contents}

1 Introduction $\quad 260$

1.1 Extremal Distributions and Polarization 262

2 Polarization and Polar Coding 266

2.1 A Basic Transform 267

2.2 An Improved Transform and Coding Scheme 269

2.3 Recursive Construction: Polarization 272

2.4 Polar Channel Coding 282

2.5 Performance 288

2.A Proof of Lemma 2.2 289

3 Complexity 292

3.1 Encoding 292

3.2 Decoding 294

3.3 Construction 295

4 Processes with Arbitrary Alphabets 307

4.1 Alphabets of Prime Size 310

4.2 Arbitrary Finite Alphabets $\quad 322$

4.3 How to Achieve Capacity 329 
$\begin{array}{lll}4.4 & \text { Complexity } & 329\end{array}$

4.A Proof of Proposition 4.8 330

4.B A Family of Polarizing Transforms 332

4.C An Alternative Proof of Polarization for Prime $q$

5 Generalized Constructions $\quad 338$

5.1 Recursive Transforms 340

5.2 Polarizing Matrices 341

5.3 Rate of Polarization 343

5.4 Proof of Theorem 5.4 349

6 Joint Polarization of Multiple Processes 354

$\begin{array}{lll}6.1 & \text { Joint Polarization } & 360\end{array}$

6.2 Rate of Polarization 365

6.A Appendix 369

7 Conclusion and Related Work 372

$\begin{array}{ll}\text { Acknowledgments } & 378\end{array}$

$\begin{array}{ll}\text { References } & 379\end{array}$ 
Foundations and Trends ${ }^{\circledR}$ in

Communications and Information Theory

Vol. 8, No. 4 (2011) 259-381

(C) 2012 E. Şaşoğlu

DOI: $10.1561 / 0100000041$

\title{
Polarization and Polar Codes
}

\author{
Eren Şaşoğlu
}

University of California, San Diego, 9500 Gilman Drive \#0436, La Jolla, CA92093-0436,USA, esasoglu@ucsd.edu

\begin{abstract}
This tutorial treats the fundamentals of polarization theory and polar coding. Arıkan's original results on binary source and channel polarization methods are studied. Error probability and complexity analyses are offered. The original results are generalized in several directions. Early developments in the field are discussed, pointers to some of the important work omitted from this tutorial are given.
\end{abstract}




\section{1}

\section{Introduction}

Figure 1.1 depicts the setting for the fundamental problem in communication theory. A sender has $K$ bits of information to send, which, after appropriate processing, are transmitted through a noisy channel that accepts input symbols one at a time and produces a sequence of output symbols. The task of the communication engineer is to design an encoding/decoding scheme that ensures that the $K$ bits are (i) transmitted in as few uses of the channel as possible, and (ii) correctly reproduced at the receiver with as high a probability as desired. In [42], Shannon showed that these seemingly conflicting requirements can be met simultaneously so long as $K$ and $N$ (the number of channel uses) are large and $K / N$ (called the rate of transmission) is below the capacity of the channel.

Shannon's proof of the channel coding theorem shows not only that reliable communication at rates below capacity is possible, but also that almost all encoding schemes, i.e., channel codes, with rates below

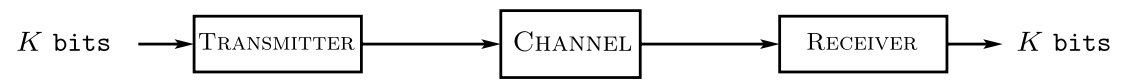

Fig. 1.1 
channel capacity will perform well as long as optimal decoders are used at the receiver. Unfortunately, optimal decoding is in general prohibitively difficult - its complexity grows exponentially in the coding length - and how to construct practical coding schemes, and especially low-complexity decoders, is not immediately clear from Shannon's coding theorem alone.

Significant progress has been made in the past sixty years toward developing practical and capacity-achieving coding methods. The bulk of the research effort to this end can be broadly divided into two groups: algebraic coding and iterative coding. Research in algebraic coding was motivated primarly by the recognition that for channels of practical interest, the words of a code must be as different from each other as possible in order to ensure their distinguishability at the receiver. Iterative codes (e.g., Turbo codes and LDPC codes), on the other hand, are designed to work well with a low-complexity decoding algorithm. Despite remarkable advances in both fields, especially in iterative coding, finding codes that (i) operate at rates close to capacity, (ii) have low computational complexity, and (iii) have provable reliability guarantees was an elusive goal until recently. ${ }^{1}$

Polar codes, invented recently by Arıkan [4], have all of these desirable properties. In particular,

- they achieve the symmetric capacity of all binary-input memoryless channels. Consequently, they are capacity-achieving for symmetric channels, which include several channel classes of practical relevance such as the binary-input additive white Gaussian noise channel, the binary symmetric channel, and the binary erasure channel.

- they are low-complexity codes, and therefore are practical: the time and space complexities of the encoding/decoding algorithms Arıkan proposes in [4] are $O(N \log N)$, where $N$ is the blocklength.

- the block error probability of polar codes is roughly $O\left(2^{-\sqrt{N}}\right)$ [9]. This performance guarantee is analytical, and is not only based on empirical evidence.

\footnotetext{
${ }^{1}$ See [12] for a historical account of the development of coding theory in general.
} 
- for symmetric channels, polar code construction is deterministic. That is, the above statements are true not only for ensembles of codes, but also for individual polar codes. Further, construction of polar codes can be accomplished with time complexity $O(N)$ and space complexity $O(\log N)$ [45].

The design philosophy of polar codes is fundamentally different from those of both algebraic codes and iterative codes (although the codes themselves are closely related to the algebraic Reed-Muller codes). It is interesting to note that the invention of these codes is the culmination of Arıkan's efforts to improve the rates achievable by convolutional codes and sequential decoding [6], a decoding method developed in the late 1950s.

The technique underlying polar codes is 'channel polarization': creating extremal channels - those that are either noiseless or useless from mediocre ones. Soon after the publication of [4], Arıkan showed that a similar technique can be used to construct optimal source codes [5] — he calls this technique 'source polarization'. It is clear in his work that a single polarization principle underlies both techniques; channel polarization and source polarization are specific applications of this principle.

\subsection{Extremal Distributions and Polarization}

Suppose we are interested in guessing (i.e., decoding) the value of a binary $N$-vector $U_{1}^{N}$ after observing a related random vector $Y_{1}^{N}$. Here, $U_{1}^{N}$ may represent a codeword chosen randomly from a channel code, and $Y_{1}^{N}$ the output of a channel when $U_{1}^{N}$ is the input. Alternatively, $U_{1}^{N}$ may be viewed as the output of a random source, and $Y_{1}^{N}$ as side information about $U_{1}^{N}$. In order to minimize the probability of decoding error, one chooses the value of $U_{1}^{N}$ that maximizes ${ }^{2}$

$$
p\left(u_{1}^{N} \mid y_{1}^{N}\right)=\prod_{i=1}^{N} p\left(u_{i} \mid y_{1}^{N}, u_{1}^{i-1}\right) .
$$

\footnotetext{
2 Throughout, we will denote probability distributions by $p$ as long as their arguments are lower case versions of the random variables they represent. For example, we will write $p(x, y \mid z)$ for $p_{X Y \mid Z}(x, y \mid z)$, denoting the joint distribution of $X$ and $Y$ conditioned on $Z$.
} 
There are two extremal cases in terms of the probability of decoding error. First, if $U_{1}^{N}$ is a function of $Y_{1}^{N}$ - i.e., if the above probability is either 0 or 1 - then its value can always be guessed correctly. Second, if $U_{1}^{N}$ is independent of $Y_{1}^{N}$ and uniformly distributed, then all guesses are equally good and will be correct with probability $1 / 2^{N}$. The first of these cases is trivial provided that the function computations can be done easily, and the second is hopeless.

A more interesting extremal case is one in which the conditional distribution of $U_{1}^{N}$ is neither $\{0,1\}$-valued nor uniform, but it is polarized in the sense that all distributions in the product formula above are either $\{0,1\}$-valued or uniform. One can view this as a case where all randomness in $U_{1}^{N}$ is concentrated in a subset of its components. Clearly, one cannot in general correctly decode such a random vector with high probability. On the other hand, decoding $U_{1}^{N}$ again becomes trivial if one has prior knowledge of its random component. The polarized structure in the probability distribution even suggests that $U_{1}^{N}$ can be decoded successively: suppose, for the sake of argument, that the odd-numbered factors in the product formula above are $\{0,1\}$-valued distributions whereas the even-numbered factors are uniform. Then, if one has prior knowledge of the even indices of $U_{1}^{N}$, then the odd indices can be determined in increasing order as follows. The decoder first computes $U_{1}$ as a function of $Y_{1}^{N}$, then produces $U_{2}$ (which is already available to it) then uses its knowledge of $U_{1}$ and $U_{2}$ to compute $U_{3}$ as a function of $\left(Y_{1}^{N}, U_{1}^{2}\right)$, etc.

A realistic model of the input/output process of a noisy channel or the output/side information process of a data source rarely fits this description. On the other hand, one may attempt to transform the process in question into one that does fit it. This is precisely the aim of Arıkan's polarization technique. In its original form, this technique consists in combining two identically distributed binary random variables so as to create two disparate random variables and repeating this operation several times to amplify the disparity, eventually approaching a polarized set of random variables. We will see this technique along with how to apply it to channel and source coding in Section 2. In Section 3 we will review the complexity of polar encoding, decoding, and code construction. As we have already mentioned, the practical appeal of 
polar codes is due to the low complexity requirements of these tasks along with provable reliability guarantees.

There has been considerable amount of research effort in polarization theory and polar coding since the publication of [4] in 2009. Arguably the main reason for this interest is the technique's ease of applicability to settings other than binary source and channel coding. In the rest of this monograph (Sections 4-6), we will review some of the main generalizations of the theory. We will begin in Section 4 by studying how discrete memoryless processes of arbitrary alphabet sizes, not just binary ones, can be polarized by recursive transforms. We will see that this can be accomplished through a linear transform similar to Arıkan's when the alphabet size is prime. Interestingly, linear transforms lose their ability to polarize all stationary memoryless processes when the underlying alphabet size is not a prime number. There are, however, non-linear transforms that do polarize all stationary memoryless processes for all finite alphabet sizes. In Section 4.2 we will study sufficient conditions for a recursive transform to polarize all such processes, and give an example of a family of transforms that satisfy these conditions for all finite alphabet sizes. The complexity and the error probability behavior of codes obtained by such transforms will be as in the binary case.

While the error probability guarantees of polar codes are unprecedented, it is of interest to know whether even stronger codes can be obtained by combining more than two random variables in each recursion of a polarizing construction. This study is undertaken in Section 5: we will first show that a large class of recursive linear transforms that combine several random variables at a time polarize memoryless processes with prime alphabet sizes. We will then characterize how a single recursion of a given polarizing transform affects error probability behavior, from which results on the large-blocklength behavior follow easily. The implications of this characterization are of a mixed nature: while in the binary case one cannot improve on the $O\left(2^{-\sqrt{N}}\right)$ error probability decay by combining a small number of random variables at a time, strong improvements become possible as the alphabet size grows.

In Section 6, we will make use of the polarization theorems of earlier sections to study joint polarization of multiple processes. We will see 
that recursive transforms, applied separately to multiple processes, not only polarize the individual processes, but the correlations between the processes are also polarized. These results will immediately lead to polar coding theorems for two-user settings such as the separate encoding of correlated sources and the multiple-access channel. 
In this section, we will review the polarization method for binary memoryless processes and show how it can be used to obtain channel and source codes that achieve optimal rates. Owing to the recursive nature of these codes, the techniques for analyzing their performance (rate, error probability, complexity) are fairly simple. In the subsequent sections we will frequently invoke the techniques discussed here. This section is based entirely on [4], [5], and [9].

Consider a pair of discrete random variables $(X, Y)$ with $X \in$ $\{0,1\}$ and $Y \in \mathcal{Y}$. The alphabet $\mathcal{Y}$ and the joint distribution of $(X, Y)$ may be arbitrary. Suppose we are given $N$ independent copies $\left(X_{1}, Y_{1}\right),\left(X_{2}, Y_{2}\right), \ldots,\left(X_{N}, Y_{N}\right)$ of $(X, Y)$. We may view $X_{1}^{N}$ as the output of a binary memoryless source, and $Y_{1}^{N}$ as side information about $X_{1}^{N}$. Alternatively, we may interpret $X_{1}^{N}$ as independent and identically distributed (i.i.d.) inputs to a binary-input memoryless channel, and $Y_{1}^{N}$ as the corresponding output. We will initially focus on the first of these interpretations and discuss the second shortly.

Suppose that a receiver observes $Y_{1}^{N}$ and is interested in decoding $X_{1}^{N}$. We know that in addition to $Y_{1}^{N}$, it is necessary and sufficient to provide the receiver with approximately $H\left(X_{1}^{N} \mid Y_{1}^{N}\right)=N H\left(X_{1} \mid Y_{1}\right)$ 
bits of information ${ }^{1}$ about $X_{1}^{N}$ for it to decode with small error probability. As we mentioned in Section 1, there are two cases where decoding is a trivial task: First, if $H\left(X_{1} \mid Y_{1}\right)=0$, the receiver can decode $X_{1}^{N}$ with no other information than $Y_{1}^{N}$ and make no errors. Second, if $H\left(X_{1} \mid Y_{1}\right)=1$, any strategy short of providing $X_{1}^{N}$ itself to the receiver - this would render the receiver's task trivial - will result in unreliable decoding.

Arıkan's polarization technique is a method that transforms the $X_{1}^{N}$ sequence so as to reduce the decoder's task into a series of these two trivial tasks. While any good source or channel code can in fact be thought of in this way, ${ }^{2}$ Arıkan's technique yields low-complexity encoding and decoding algorithms due to its recursive nature.

\subsection{A Basic Transform}

In this section we review a single step of the polarization technique. Although the reader may find some of the details here trivial, we find it worthwhile to go through them since most polarization ideas are contained in the one-step construction.

Consider the case $N=2$. Given $\left(X_{1}, Y_{1}\right)$ and $\left(X_{2}, Y_{2}\right)$, we define $S_{1}, S_{2} \in\{0,1\}$ through the mapping (see Figure 2.1)

$$
S_{1}=X_{1}+X_{2} \quad \text { and } \quad S_{2}=X_{2}
$$

where ' + ' denotes modulo-2 addition. Notice that the correspondence between $S_{1}, S_{2}$ and $X_{1}, X_{2}$ is one-to-one, and therefore the

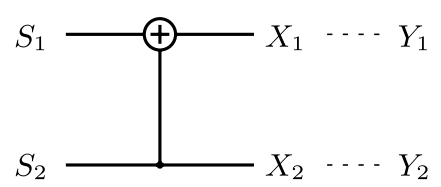

Fig. 2.1 The first step of the recursive construction. The distribution on $\left(S_{1}, S_{2}\right)$ is induced by the distribution on $\left(X_{1}^{2}, Y_{1}^{2}\right)$.

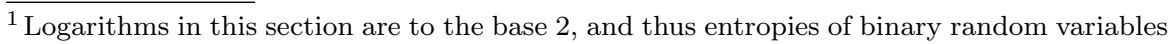
are $[0,1]$-valued.

${ }^{2} \mathrm{~A}$ brief discussion on this is offered in the introduction of Section 5.
} 
independence of $\left(X_{1}, Y_{1}\right)$ and $\left(X_{2}, Y_{2}\right)$ implies

$$
2 H\left(X_{1} \mid Y_{1}\right)=H\left(S_{1}^{2} \mid Y_{1}^{2}\right)=H\left(S_{1} \mid Y_{1}^{2}\right)+H\left(S_{2} \mid Y_{1}^{2} S_{1}\right) .
$$

It easily follows from (2.1) and the above equalities that

$$
H\left(S_{2} \mid Y_{1}^{2} S_{1}\right) \leq H\left(X_{1} \mid Y_{1}\right) \leq H\left(S_{1} \mid Y_{1}^{2}\right) .
$$

Due to these entropy relations, one intuitively expects that observing $\left(Y_{1}^{2} S_{1}\right)$ yields a more reliable estimate of $S_{2}$ (i.e., $\left.X_{2}\right)$ than observing $Y_{2}$ alone does. (It is in fact clear that the 'channel' $S_{2} \rightarrow Y_{1}^{2} S_{1}$ is upgraded with respect to the channel $X_{2} \rightarrow Y_{2}$.) Similarly, observing $Y_{1}^{2}$ alone leads to a less reliable estimate of $S_{1}$. If we let $P_{\mathrm{e}}\left(X_{1} \mid Y_{1}\right)$ denote the average error probability of optimally decoding $X_{1}$ by observing $Y_{1}$, we indeed have

$$
P_{\mathrm{e}}\left(S_{2} \mid Y_{1}^{2} S_{1}\right) \leq P_{\mathrm{e}}\left(X_{1} \mid Y_{1}\right) \leq P_{\mathrm{e}}\left(S_{1} \mid Y_{1}^{2}\right) .
$$

The left-hand inequality above is obtained through the relations

$$
P_{\mathrm{e}}\left(S_{2} \mid Y_{1}^{2} S_{1}\right) \leq P_{\mathrm{e}}\left(S_{2} \mid Y_{2}\right)=P_{\mathrm{e}}\left(X_{1} \mid Y_{1}\right)
$$

and the right-hand inequality through

$$
\begin{aligned}
P_{\mathrm{e}}\left(X_{1} \mid Y_{1}\right) & =P_{\mathrm{e}}\left(X_{1}+X_{2} \mid Y_{1} X_{2}\right) \\
& =P_{\mathrm{e}}\left(X_{1}+X_{2} \mid Y_{1}^{2} X_{2}\right) \\
& \leq P_{\mathrm{e}}\left(X_{1}+X_{2} \mid Y_{1}^{2}\right) .
\end{aligned}
$$

The second equality above is due to the Markov chain $\left(X_{1}+X_{2}\right)$ $Y_{1} X_{2}-Y_{2}$

One can see the use of these relations in the following coding scheme: upon observing $X_{1}^{2}$, the encoder computes $S_{1}^{2}$ and reveals $S_{1}$ to the receiver. The receiver then uses the optimal decision rule to decode $S_{2}$ from $\left(Y_{1}^{2} S_{1}\right)$, and computes $\left(\hat{X}_{1}, \hat{X}_{2}\right)=\left(S_{1}+\hat{S}_{2}, \hat{S}_{2}\right)$, where $\hat{S}_{2}$ is its estimate of $S_{2}$.

This is in fact the simplest instance of polar source coding, with code blocklength 2 , rate $1 / 2$, and average block error probability $P_{\mathrm{e}}\left(S_{2} \mid Y_{1}^{2} S_{1}\right)$. Simple as it is, this scheme contains the essence of polarization and polar coding ideas: out of two identical entropy terms 
$H\left(X_{1} \mid Y_{1}\right)$ and $H\left(X_{2} \mid Y_{2}\right)$, we have created two different entropies one of which is closer to 0 than the original and the other closer to 1 , thereby approaching (albeit not very closely) the trivial cases we mentioned above. By revealing to the decoder those random variables with high conditional entropies, we can decode with higher reliability those that have lower entropies.

\subsection{An Improved Transform and Coding Scheme}

Since the random variables $S_{1}$ and $S_{2}$ created by the above transform are $\{0,1\}$-valued, one can apply the same transform to these in order to enhance the disparity between their entropies. How this can be done is depicted in Figure 2.2: set $N=4$ and define, in addition to $S_{1}, S_{2}$ in $(2.1)$,

$$
T_{1}=X_{3}+X_{4} \quad \text { and } \quad T_{2}=X_{4},
$$

and also define $\tilde{Y}_{1}=Y_{1}^{2}$ and $\tilde{Y}_{2}=Y_{3}^{4}$. Observe that $\left(S_{1}, \tilde{Y}_{1}\right)$ and $\left(T_{1}, \tilde{Y}_{2}\right)$ are i.i.d., just as were $\left(X_{1}, Y_{1}\right)$ and $\left(X_{2}, Y_{2}\right)$. It then follows similarly to (2.2) that

$$
H\left(T_{1} \mid \tilde{Y}_{1}^{2}, S_{1}+T_{1}\right) \leq H\left(S_{1} \mid \tilde{Y}_{1}\right) \leq H\left(S_{1}+T_{1} \mid \tilde{Y}_{1}^{2}\right) .
$$

Similarly, defining $\bar{Y}_{1}=\left(Y_{1}^{2} S_{1}\right)$ and $\bar{Y}_{2}=\left(Y_{3}^{4} T_{1}\right)$ and noting that $\left(S_{2}, \bar{Y}_{1}\right)$ and $\left(T_{2}, \bar{Y}_{2}\right)$ are also i.i.d., we have

$$
H\left(T_{2} \mid \bar{Y}_{1}^{2}, S_{2}+T_{2}\right) \leq H\left(S_{2} \mid \bar{Y}_{1}\right) \leq H\left(S_{2}+T_{2} \mid \bar{Y}_{1}^{2}\right) .
$$

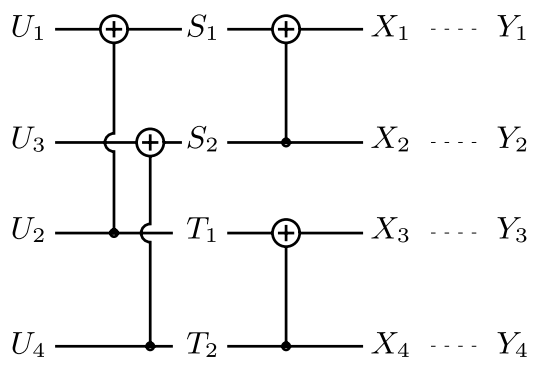

Fig. 2.2 Recursive two-step transform. 
The relevance of the entropy terms above can be seen by an inspection of Figure 2.2. In particular, we have

$$
\begin{aligned}
4 H\left(X_{1} \mid Y_{1}\right)= & 2 H\left(S_{1}^{2} \mid Y_{1}^{2}\right) \\
= & H\left(U_{1}^{4} \mid Y_{1}^{4}\right) \\
= & H\left(U_{1} \mid Y_{1}^{4}\right)+H\left(U_{2} \mid Y_{1}^{4} U_{1}\right) \\
& +H\left(U_{3} \mid Y_{1}^{4} U_{1}^{2}\right)+H\left(U_{4} \mid Y_{1}^{4} U_{1}^{3}\right) .
\end{aligned}
$$

It is also easily seen that the last four entropy terms above are those appearing in (2.4) and (2.5):

$$
\begin{aligned}
H\left(U_{1} \mid Y_{1}^{4}\right) & =H\left(S_{1}+T_{1} \mid \tilde{Y}_{1}^{2}\right) \\
H\left(U_{2} \mid Y_{1}^{4} U_{1}\right) & =H\left(T_{1} \mid \tilde{Y}_{1}^{2}, S_{1}+T_{1}\right) \\
H\left(U_{3} \mid Y_{1}^{4} U_{1}^{2}\right) & =H\left(S_{2}+T_{2} \mid Y_{1}^{4} S_{1} T_{1}\right)=H\left(S_{2}+T_{2} \mid \bar{Y}_{1}^{2}\right) \\
H\left(U_{4} \mid Y_{1}^{4} U_{1}^{3}\right) & =H\left(T_{2} \mid Y_{1}^{4} S_{1} T_{1}, S_{2}+T_{2}\right)=H\left(T_{2} \mid \bar{Y}_{1}^{2}, S_{2}+T_{2}\right) .
\end{aligned}
$$

It follows from these relations, along with (2.4) and (2.5), that

$$
\begin{aligned}
& H\left(U_{2} \mid Y_{1}^{4} U_{1}\right) \leq H\left(S_{1} \mid Y_{1}^{2}\right) \leq H\left(U_{1} \mid Y_{1}^{4}\right) \\
& H\left(U_{4} \mid Y_{1}^{4} U_{1}^{3}\right) \leq H\left(S_{2} \mid Y_{1}^{2} S_{1}\right) \leq H\left(U_{3} \mid Y_{1}^{4} U_{1}^{2}\right) .
\end{aligned}
$$

That is, from the two entropy terms $H\left(S_{1} \mid Y_{1}^{2}\right)$ and $H\left(S_{2} \mid Y_{1}^{2} S_{1}\right)$ we obtain four new entropies that are separated from the original two as in the above inequalities. There is no general inequality between $H\left(U_{2} \mid Y_{1}^{4} U_{1}\right)$ and $H\left(U_{3} \mid Y_{1}^{4} U_{1}^{2}\right)$. Nevertheless, since $H\left(S_{1} \mid Y_{1}^{2}\right)$ and $H\left(S_{2} \mid Y_{1}^{2} S_{1}\right)$ were already somewhat polarized toward 1 and 0 , the above inequalities say that the polarization effect is enhanced by the second application of the transform.

Consider now the following source code of blocklength 4: We choose a set $\mathcal{A} \subset\{1,2,3,4\}$ with $|\mathcal{A}|=4-k$. Upon observing $X_{1}^{4}=x_{1}^{4}$, the encoder computes $U_{1}^{4}=u_{1}^{4}$ and sends all $u_{i}, i \in \mathcal{A}^{c}$ to the decoder, therefore the rate of the code is $k / 4$ bits/symbol. The decoder outputs its estimate $\hat{u}_{1}^{4}$ of $u_{1}^{4}$ successively as

$$
\hat{u}_{i}= \begin{cases}u_{i}, & \text { if } i \in \mathcal{A}^{c} \\ 0, & \text { if } i \in \mathcal{A} \text { and } L\left(y_{1}^{4}, \hat{u}_{1}^{i-1}\right)>1, \\ 1, & \text { otherwise }\end{cases}
$$


where

$$
L\left(y_{1}^{4}, u_{1}^{i-1}\right)=\frac{p_{U_{i} \mid Y_{1}^{4} U_{1}^{i-1}}\left(0 \mid y_{1}^{4}, \hat{u}_{1}^{i-1}\right)}{p_{U_{i} \mid Y_{1}^{4} U_{1}^{i-1}}\left(1 \mid y_{1}^{4}, \hat{u}_{1}^{i-1}\right)} .
$$

The probability functions above are those that describe the entropies $H\left(U_{i} \mid Y_{1}^{4} U_{1}^{i-1}\right)$. One may therefore expect that the above scheme perform well if the set $\mathcal{A}$ consists of bits with the smallest conditional entropies (i.e., the highest reliabilities). A similar and sensible choice of set $\mathcal{A}$ is the following:

$$
i \in \mathcal{A} \text { and } j \in \mathcal{A}^{c} \text { imply } P_{\mathrm{e}}\left(U_{i} \mid Y_{1}^{4} U_{1}^{i-1}\right) \leq P_{\mathrm{e}}\left(U_{j} \mid Y_{1}^{4} U_{1}^{j-1}\right)
$$

This choice can be justified by the following result:

Proposition 2.1. The average block error probability of the above coding scheme is at most

$$
\sum_{i \in \mathcal{A}} P_{\mathrm{e}}\left(U_{i} \mid Y_{1}^{4} U_{1}^{i-1}\right)
$$

Proof. Consider a decoder with output $\tilde{u}_{1}^{N}$, whose decision rule for $\tilde{u}_{i}$ is obtained from $(2.6)$ by replacing $L\left(y_{1}^{4}, \hat{u}_{1}^{i-1}\right)$ with $L\left(y_{1}^{4}, u_{1}^{i-1}\right)$. This is a genie-aided version of the original decoder: at each step of decoding, a genie provides the decoder with the correct value of the previously decoded bits. Clearly, the average error probability of the $i$ th constituent of this decoder is $P_{\mathrm{e}}\left(U_{i} \mid Y_{1}^{4} U_{1}^{i-1}\right)$, and therefore the block error probability is upper bounded by the expression in (2.8). In order to conclude the proof, we will now show that the block error events for the original decoder described in (2.6) and (2.7) and its genie-aided version are identical.

Note that $\hat{u}_{1}=\tilde{u}_{1}$ for each realization $\left(y_{1}^{4}, u_{1}^{4}\right)$, as both decisions depend on $L\left(y_{1}^{4}\right)$ alone. Hence, if $\hat{u}_{1}=\tilde{u}_{1}=u_{1}$ (otherwise both decoders commit a block error in the first step), it then follows that $\hat{u}_{2}=\tilde{u}_{2}$, as both decisions are now based on $L\left(y_{1}^{4}, u_{1}\right)$. Continuing in this manner, we see that at each step, either both decoders have already committed an error, or their next decisions will be identical. This in turn implies that the block error events (but not necessarily the bit error events) 
under the original decoder and its genie-aided version are identical, yielding the claim.

Proposition 2.1 highlights two simple but important aspects of the design and analysis of polar codes (of which the above code is an instance). First, the block error probability behavior of these codes can be deduced from the error behavior of the created 'channels' (e.g., channels $U_{i} \rightarrow Y_{1}^{4} U_{1}^{i-1}$ above), which as we will see greatly simplifies error analysis. Second, minimizing the upper bound in (2.8) amounts to finding a good code, as it consists in determining the bit indices with the smallest probability of decoding error. This is one of the several appeals of polar codes: their design and construction on one hand and analysis on the other are closely linked and do not require separate techniques.

\subsection{Recursive Construction: Polarization}

We saw the first two steps of Arıkan's construction in the previous sections. The recursive nature of this construction is evident; the second step merely involves applying the transform in (2.1) to the random variables obtained in the first. Similarly in the general form of this construction, each recursion consists in applying (2.1) to the random variables obtained in the previous one. For this technique to create the desired effect of driving the entropies close to 0 and 1 , it is therefore necessary that the basic transform in (2.1) lead to a strict separation of entropies, i.e., that the inequalities in (2.2) be strict, for otherwise the transform would have no effect. The following result guarantees that this requirement is always met, except in trivial cases.

Lemma 2.2. Let $\left(X_{1}, Y_{1}\right)$ and $\left(X_{2}, Y_{2}\right)$ be independent pairs of discrete random variables with $X_{1}, X_{2} \in\{0,1\}, H\left(X_{1} \mid Y_{1}\right)=\alpha$, and $H\left(X_{2} \mid\right.$ $\left.Y_{2}\right)=\beta$ for some $\alpha, \beta \in[0,1]$. The entropy $H\left(X_{1}+X_{2} \mid Y_{1}^{2}\right)$

(i) is minimized when $H\left(X_{1} \mid Y_{1}=y_{1}\right)=\alpha, H\left(X_{2} \mid Y_{2}=y_{2}\right)=\beta$ for all $y_{1}, y_{2}$ with $p\left(y_{1}\right), p\left(y_{2}\right)>0$.

(ii) is maximized when $H\left(X_{1} \mid Y_{1}=y_{1}\right), H\left(X_{2} \mid Y_{2}=y_{2}\right) \in\{0,1\}$ for all $y_{1}, y_{2}$ with $p\left(y_{1}\right), p\left(y_{2}\right)>0$. 
It also follows from (i) that if $\alpha, \beta \in(\delta, 1-\delta)$ for some $\delta>0$, then there exists $\epsilon(\delta)>0$ such that

$$
H\left(X_{1}+X_{2} \mid Y_{1}^{2}\right)-H\left(X_{1} \mid Y_{1}\right) \geq \epsilon(\delta) .
$$

Proof. See Appendix 2.A.

We can now describe the general form of the polarization construction: let $\left(X_{1}, Y_{1}\right),\left(X_{2}, Y_{2}\right), \ldots$ be an i.i.d. sequence as above. For $n=$ $0,1, \ldots$, set $N=2^{n}$ and define a sequence of transforms $G_{n}:\{0,1\}^{N} \rightarrow$ $\{0,1\}^{N}$ recursively through

$$
\begin{aligned}
G_{0}(u) & =u, \\
G_{n}\left(u_{1}, u_{2}\right) & =\pi_{n}\left(G_{n-1}\left(u_{1}\right)+G_{n-1}\left(u_{2}\right), G_{n-1}\left(u_{2}\right)\right), \quad n=1,2, \ldots
\end{aligned}
$$

where $u_{1}, u_{2} \in\{0,1\}^{N / 2}$ and $\pi_{n}:\{0,1\}^{N} \rightarrow\{0,1\}^{N}$ permutes the components of its argument vector through

$$
\begin{aligned}
& \pi_{n}(v)_{2 i-1}=v_{i} \\
& \pi_{n}(v)_{2 i}=v_{i+N / 2}
\end{aligned}, \quad i=1, \ldots, N / 2 .
$$

It is easy to show [4] that $G_{n}$ is one-to-one and that $G_{n}^{-1}=G_{n}$. Now define

$$
U_{1}^{N}=G_{n}\left(X_{1}^{N}\right)
$$

The general form of the transform $G_{n}$ is shown in Figure 2.3. The inclusion of $\pi_{n}$ in the definition of $G_{n}$ is not necessary for the polarization technique to work, but it will greatly simplify the notation. One can verify that $G_{1}$ and $G_{2}$ are equivalent to the transforms in the previous sections (Figures 2.1 and 2.2).

The main result in [4] and [5] is that as the construction size $N$ grows, the entropies $H\left(U_{i} \mid Y_{1}^{N} U_{1}^{i-1}\right)$ approach either 0 or 1 :

Theorem 2.3. For all $\epsilon>0$,

$$
\begin{aligned}
& \lim _{n \rightarrow \infty} \frac{1}{N}\left|\left\{i: H\left(U_{i} \mid Y_{1}^{N} U_{1}^{i-1}\right)>1-\epsilon\right\}\right|=H(X \mid Y), \\
& \lim _{n \rightarrow \infty} \frac{1}{N}\left|\left\{i: H\left(U_{i} \mid Y_{1}^{N} U_{1}^{i-1}\right)<\epsilon\right\}\right|=1-H(X \mid Y) .
\end{aligned}
$$




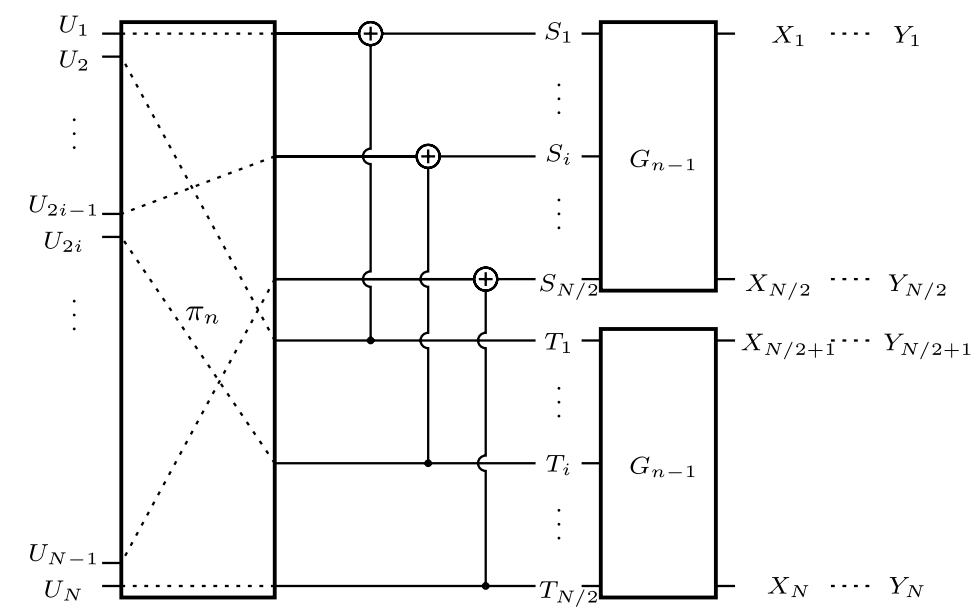

Fig. 2.3 Two copies of the $(n-1)$-step polarization transform $G_{n-1}$ are combined to obtain the $n$-step transform $G_{n}$.

In order to simplify the notation in the proofs, we will often use the following definition.

Definition 2.1. For i.i.d. $\left(X_{1}, Y_{1}\right)$ and $\left(X_{2}, Y_{2}\right)$ with $H:=H\left(X_{1} \mid Y_{1}\right)$, we define

$$
\begin{aligned}
& H^{-}:=H\left(X_{1}+X_{2} \mid Y_{1}^{2}\right), \\
& H^{+}:=H\left(X_{2} \mid Y_{1}^{2}, X_{1}+X_{2}\right) .
\end{aligned}
$$

With the above definitions, we claim that

$$
\begin{aligned}
H\left(U_{1} \mid Y_{1}^{N}\right) & =H^{-\cdots--} \\
H\left(U_{2} \mid Y_{1}^{N} U_{1}\right) & =H^{-\cdots-+} \\
H\left(U_{3} \mid Y_{1}^{N} U_{1}^{2}\right) & =H^{-\cdots+-} \\
& \vdots \\
H\left(U_{N-1} \mid Y_{1}^{N} U_{1}^{N-2}\right) & =H^{+\cdots+-} \\
H\left(U_{N} \mid Y_{1}^{N} U_{1}^{N-1}\right) & =H^{+\cdots++},
\end{aligned}
$$

where the superscripts on the right-hand terms are of length $n$. These equivalences can be verified by an inspection of Figure 2.3. In particular, let us suppose that the equalities in (2.10) hold for the entropy 
terms obtained after $G_{n-1}$, so that for every $1 \leq i \leq N / 2$ there is a distinct $\mathbf{s} \in\{-,+\}^{n-1}$ such that $H\left(S_{i} \mid Y_{1}^{N / 2} S_{1}^{i-1}\right)=H^{\mathbf{s}}$. Then, since the pairs $\left(S_{i}, Y_{1}^{N / 2} S_{1}^{i-1}\right)$ and $\left(T_{i}, Y_{N / 2+1}^{N} T_{1}^{i-1}\right)$ in the figure are i.i.d., it is easily seen that $H\left(U_{2 i-1} \mid Y_{1}^{N} U_{1}^{2 i-2}\right)=H\left(S_{i} \mid Y_{1}^{N / 2} S_{1}^{i-1}\right)^{-}=H^{\mathbf{s}-}$, and that $H\left(U_{2 i} \mid Y_{1}^{N} U_{1}^{2 i-1}\right)=H\left(S_{i} \mid Y_{1}^{N / 2} S_{1}^{i-1}\right)^{+}=H^{\mathbf{s}+}$. It follows that for every $i \in\{1, \ldots, N\}$ there is a distinct $\mathbf{s} \in\{-,+\}^{n}$ such that $H\left(U_{i} \mid\right.$ $\left.Y_{1}^{N} U_{1}^{i-1}\right)=H^{\mathrm{s}}$. It also follows from the definition of the permutation function $\pi_{n}$ that these equivalences are as in (2.10). Since we have already seen in Section 2.1 that (2.10) holds for $n=1$, it follows by induction that it holds for all $n$.

In order to prove Theorem 2.3 we define an i.i.d. process $B_{1}, B_{2}, \ldots$ where $B_{1}$ is uniformly distributed over $\{-,+\}$. We then define a $[0,1]$ valued random process $H_{0}, H_{1}, \ldots$ recursively as

$$
\begin{aligned}
& H_{0}=H\left(X_{1} \mid Y_{1}\right), \\
& H_{n}=H_{n-1}^{B_{n}}, \quad n=1,2, \ldots
\end{aligned}
$$

As $B_{1}, \ldots, B_{n}$ is uniformly distributed over $\{-,+\}^{n}$, the entropy equivalences in (2.10) imply that for all $n$,

$$
\operatorname{Pr}\left[H_{n} \in \mathcal{I}\right]=\frac{1}{N}\left|\left\{i: H\left(U_{i} \mid Y_{1}^{N} U_{1}^{i-1}\right) \in \mathcal{I}\right\}\right|
$$

for any $\mathcal{I} \subseteq[0,1]$. Therefore, Theorem 2.3 is implied by

Theorem 2.4. $H_{n}$ converges almost surely to a $\{0,1\}$-valued random variable $H_{\infty}$ with $\operatorname{Pr}\left[H_{\infty}=1\right]=1-\operatorname{Pr}\left[H_{\infty}=0\right]=H\left(X_{1} \mid Y_{1}\right)$.

Proof. Definitions (2.9) and (2.11) imply that $H_{n}^{-}+H_{n}^{+}=2 H_{n}$. It follows that the process $H_{1}, H_{2}, \ldots$ is a bounded martingale and therefore converges almost surely to a random variable $H_{\infty}$. As almost sure convergence implies convergence in $\mathcal{L}^{1}$, we have $E\left[\mid H_{n+1}-\right.$ $\left.H_{n} \mid\right]=\frac{1}{2} E\left[H_{n}^{-}-H_{n}\right]+\frac{1}{2} E\left[H_{n}-H_{n}^{+}\right]=E\left[H_{n}^{-}-H_{n}\right] \rightarrow 0$. Also since Lemma 2.2 implies that $H_{n}^{-}-H_{n}>\delta(\epsilon)$ if $H_{n} \in(\epsilon, 1-\epsilon)$, it follows that $H_{n} \rightarrow\{0,1\}$ with probability 1 , i.e., that $H_{\infty}$ is $\{0,1\}$-valued. The claim on the distribution of $H_{\infty}$ then follows from the relation $E\left[H_{\infty}\right]=E\left[H_{0}\right]=H\left(X_{1} \mid Y_{1}\right)$. 
This is the main polarization theorem. It states that Arıkan's construction distills the randomness in an i.i.d. binary process into a sequence of uniform or constant binary random variables. Equivalently, this construction can be interpreted as one that creates a sequence of noiseless and useless channels $U_{i} \rightarrow Y_{1}^{N} U_{1}^{i-1}$ out of several copies of a memoryless channel $X_{1} \rightarrow Y_{1}$.

Theorem 2.3 can be exploited to construct entropy-achieving polar source codes as follows: Fix $\delta, \epsilon>0$ and find the set

$$
\mathcal{A}:=\left\{i: P_{\mathrm{e}}\left(U_{i} \mid Y_{1}^{N} U_{1}^{i-1}\right) \leq \epsilon\right\} .
$$

As $H\left(U_{i} \mid Y_{1}^{N} U_{1}^{i-1}\right) \rightarrow 0$ implies $P_{\mathrm{e}}\left(U_{i} \mid Y_{1}^{N} U_{1}^{i-1}\right) \rightarrow 0$, it follows from Theorem 2.3 that $\mathcal{A}$ must be of size at least $(1-H(X \mid Y)-\delta) N$ provided that the blocklength $N$ is sufficiently large. The encoder observes $X_{1}^{N}$, computes $U_{1}^{N}=G_{n}\left(X_{1}^{N}\right)$, and reveals $U_{i}, i \in \mathcal{A}^{c}$ to the receiver, therefore the code is of rate $H(X \mid Y)+\delta$. Upon observing $Y_{1}^{N}$ and $U_{i}, i \in \mathcal{A}^{c}$, the receiver decodes $U_{1}^{N}$ successively as in (2.6) and (2.7). Similarly to the previous section, the block error probability of this code is at most

$$
\sum_{i \in \mathcal{A}} P_{\mathrm{e}}\left(U_{i} \mid Y_{1}^{N} U_{1}^{i-1}\right) \leq \epsilon N
$$

This bound on the error probability is not very useful, however, as we have chosen the threshold $\epsilon$ independently of $N$. Fortunately, the choice of set $\mathcal{A}$ in the above scheme can be modified slightly to include a blocklength-dependent $\epsilon$, yielding codes with vanishing block error probability. More precisely, instead of $\mathcal{A}$ consider the set

$$
\mathcal{A}_{\beta}^{\prime}:=\left\{i: P_{\mathrm{e}}\left(U_{i} \mid Y_{1}^{N} U_{1}^{i-1}\right) \leq 2^{-N^{\beta}}\right\}
$$

for some $\beta>0$. Note that for large $N$ we have $\mathcal{A}_{\beta}^{\prime} \subset \mathcal{A}$. The next result states that as long as $\beta<1 / 2$, the set difference $\mathcal{A} \backslash \mathcal{A}_{\beta}^{\prime}$ is negligibly small, in the sense that $\left|\mathcal{A}_{\beta}^{\prime}\right| /|\mathcal{A}| \rightarrow 1$. That is, at large blocklengths if the bit error probability $P_{\mathrm{e}}\left(U_{i} \mid Y_{1}^{N} U_{1}^{i-1}\right)$ is small, then it must indeed be exponentially small in the square root of the blocklength. 
Theorem 2.5. For all $\beta<1 / 2$ and $\delta>0$, there exists $N_{o}=N_{o}(\beta, \delta)$ such that

$$
\left|\mathcal{A}_{\beta}^{\prime}\right|>(1-H(X \mid Y)-\delta) N
$$

for all $N \geq N_{o}$.

Corollary 2.6. For all $\beta<1 / 2$ and rates strictly above $H(X \mid Y)$, the average block error probability of the above source coding scheme is $o\left(2^{-N^{\beta}}\right)$.

In order to prove Theorem 2.5 one needs to compute the error probability terms $P_{\mathrm{e}}\left(U_{i} \mid Y_{1}^{N} U_{1}^{i-1}\right)$ that emerge during the polarization process. The difficulty in doing so is that the joint distributions of $\left(U_{i}, Y_{1}^{N} U_{1}^{i-1}\right)$ become increasingly complex as the blocklength grows, and consequently the exact computation of error probabilities becomes intractible. One may hope instead to find useful bounds on the error probabilities that are also independent of the details of the joint distributions. For this purpose, consider a $[0,1]$-valued parameter $Z(X \mid Y)$ defined as

$$
Z(X \mid Y)=2 \sum_{y \in \mathcal{Y}} \sqrt{p_{X Y}(0, y) p_{X Y}(1, y)} .
$$

Arıkan calls $Z(X \mid Y)$ the source Bhattacharyya parameter [5]. It is well-known that the Bhattacharyya parameter upper bounds the error probability of the optimal decision rule, and therefore may be used as a measure of reliability:

Proposition 2.7. $P_{\mathrm{e}}(X \mid Y) \leq Z(X \mid Y)$.

Proof.

$$
\begin{aligned}
P_{\mathrm{e}}(X \mid Y) \leq & p_{X}(0) \sum_{y} p(y \mid 0) \mathbb{1}_{[p(0 \mid y) \leq p(1 \mid y)]} \\
& +p_{X}(1) \sum_{y} p(y \mid 1) \mathbb{1}_{[p(1 \mid y) \leq p(0 \mid y)]}
\end{aligned}
$$




$$
\begin{aligned}
& \leq p_{X}(0) \sum_{y} \frac{p(0 \mid y) p(y)}{p_{X}(0)} \frac{\sqrt{p(1 \mid y)}}{\sqrt{p(0 \mid y)}} \\
& \quad+p_{X}(1) \sum_{y} \frac{p(1 \mid y) p(y)}{p_{X}(1)} \frac{\sqrt{p(0 \mid y)}}{\sqrt{p(1 \mid y)}} \\
& =2 \sum_{y} \sqrt{p(0, y) p(1, y)} \\
& =Z(X \mid Y) .
\end{aligned}
$$

As a measure of reliability, it would be natural for $Z(X \mid Y)$ to satisfy

$$
\begin{aligned}
& Z(X \mid Y) \approx 1 \text { if and only if } H(X \mid Y) \approx 1, \\
& Z(X \mid Y) \approx 0 \text { if and only if } H(X \mid Y) \approx 0 .
\end{aligned}
$$

The following relations show that this is indeed the case:

Proposition 2.8.

$$
\begin{aligned}
Z(X \mid Y)^{2} & \leq H(X \mid Y) \\
H(X \mid Y) & \leq \log (1+Z(X \mid Y))
\end{aligned}
$$

We defer the proof until Section 4, where we show a more general result for a generalized definition of the Bhattacharyya parameter (Proposition 4.8).

One may expect to observe a disparity between the Bhattacharyya parameters after one step of the polarization transform, similar to the disparity between the entropies (2.2) and the error probabilities (2.3). We indeed have

$$
Z\left(U_{2} \mid Y_{1}^{2} U_{1}\right) \leq Z\left(X_{1} \mid Y_{1}\right) \leq Z\left(U_{1} \mid Y_{1}^{2}\right) .
$$

The reader can verify that these inequalities are strict unless $Z\left(X_{1} \mid Y_{1}\right)$ is either 0 or 1 . Clearly, the exact values of these parameters depend on the details of the joint distribution of $\left(X_{1}, Y_{1}\right)$. Nevertheless, there are bounds on these that are distribution-independent and are also sufficiently good for proving Theorem 2.5: 
Lemma 2.9. For all $\left(X_{1}, Y_{1}\right)$, we have

$$
\begin{aligned}
Z\left(U_{1} \mid Y_{1}^{2}\right) & \leq 2 Z\left(X_{1} \mid Y_{1}\right), \\
Z\left(U_{2} \mid Y_{1}^{2} U_{1}\right) & =Z\left(X_{1} \mid Y_{1}\right)^{2} .
\end{aligned}
$$

Proof. First note that $p\left(u_{1}, u_{2}, y_{1}, y_{2}\right)=p_{X Y}\left(u_{1}+u_{2}, y_{1}\right) p_{X Y}\left(u_{2}, y_{2}\right)$. The first bound can be seen through the following inequalities:

$$
\begin{array}{r}
Z\left(U_{1} \mid Y_{1}^{2}\right)=2 \sum_{y_{1}^{2}}\left[\sum_{u_{2}} p_{X Y}\left(u_{2}, y_{1}\right) p_{X Y}\left(u_{2}, y_{2}\right)\right. \\
\left.\cdot \sum_{v_{2}} p_{X Y}\left(1+v_{2}, y_{1}\right) p_{X Y}\left(v_{2}, y_{2}\right)\right]^{1 / 2} \\
\leq 2 \sum_{y_{1}^{2}, u_{2}, v_{2}}\left[p_{X Y}\left(u_{2}, y_{1}\right) p_{X Y}\left(1+v_{2}, y_{1}\right)\right. \\
\left.=2 \sum_{u_{2}, v_{2}} \sum_{y_{1}}\left[p_{X Y}\left(u_{X}, y_{1}\right) p_{X Y}\left(1+y_{2}\right) p_{X Y}\left(v_{2}, y_{2}\right)\right]^{1 / 2}\right]^{1 / 2} \\
\cdot \sum_{y_{2}}\left[p_{X Y}\left(u_{2}, y_{2}\right) p_{X Y}\left(v_{2}, y_{2}\right)\right]^{1 / 2}
\end{array}
$$

The term inside the outermost summation is equal to $p\left(u_{2}\right) Z\left(X_{1} \mid Y_{1}\right) / 2$ for all $u_{2}, v_{2}$. This yields the first claim. To obtain the second claim we write

$$
\begin{aligned}
& Z\left(U_{2} \mid Y_{1}^{2} U_{1}\right)= 2 \sum_{y_{1}^{2}, u_{1}}\left[p_{X Y}\left(u_{1}, y_{1}\right) p_{X Y}\left(0, y_{2}\right)\right. \\
&\left.\cdot p_{X Y}\left(u_{1}+1, y_{1}\right) p_{X Y}\left(1, y_{2}\right)\right]^{1 / 2} \\
&=2 \sum_{u_{1}} \sum_{y_{1}}\left[p_{X Y}\left(u_{1}, y_{1}\right) p_{X Y}\left(u_{1}+1, y_{1}\right)\right]^{1 / 2} \\
& \cdot \sum_{y_{2}}\left[p_{X Y}\left(0, y_{2}\right) p_{X Y}\left(1, y_{2}\right)\right]^{1 / 2}
\end{aligned}
$$




$$
\begin{aligned}
& =4\left[\sum_{y}\left[p_{X Y}(0, y) p_{X Y}(1, y)\right]^{1 / 2}\right]^{2} \\
& =Z\left(X_{1} \mid Y_{1}\right)^{2} .
\end{aligned}
$$

In order to prove Theorem 2.5, we will define, similarly to the proof of Theorem 2.3, a random process that mirrors the behavior of the Bhattacharyya parameters obtained during the polarization construction. For this purpose, we first let $Z:=Z\left(X_{1} \mid Y_{1}\right)$ and define

$$
\begin{aligned}
& Z^{-}:=Z\left(U_{1} \mid Y_{1}^{2}\right), \\
& Z^{+}:=Z\left(U_{2} \mid Y_{1}^{2} U_{1}\right) .
\end{aligned}
$$

We will see that bounds (2.12) and (2.13) on $Z^{-}$and $Z^{+}$suffice to prove Theorem 2.5. To get an initial idea about the reason for this, let us neglect, for a moment, the factor 2 in the bound $(2.12)$ on $Z^{-}$. It is now easy to see that on a 'polarization path' consisting of $n$ consecutive '+' and ' - ' operations, the resulting $Z\left(U_{i} \mid Y_{1}^{N} U_{1}^{i-1}\right)$ will be upper bounded by $Z(X \mid Y)^{2^{n} p}$, where $n_{p}$ is the number of the occurrences of ' + '. Since on a typical path the plus and the minus operations occur with roughly the same frequency, i.e., $n_{p} \approx n / 2$, it follows that most Bhattacharyya parameters will be of the form $Z\left(U_{i} \mid Y_{1}^{N} U_{1}^{i-1}\right) \approx Z(X \mid$ $Y)^{2^{n / 2}}=Z(X \mid Y)^{\sqrt{N}}$, as claimed in Theorem 2.5.

The reason for us to resort to Bhattacharyya parameters instead of working directly with error probabilities is the lack of useful bounds on the latter. More precisely, although we have

$$
P_{\mathrm{e}}\left(U_{2} \mid Y_{1}^{2} U_{1}\right) \leq P_{\mathrm{e}}\left(X_{1} \mid Y_{1}\right) \leq P_{\mathrm{e}}\left(U_{1} \mid Y_{1}^{2}\right)
$$

after the first step of polarization, how close these error terms are to each other depends strongly on the distribution of $\left(X_{1}, Y_{1}\right)$. In particular, it can easily be verified that if $X_{1}$ is uniformly distributed and $Y_{1}$ is the output of a binary symmetric channel whose input is $X_{1}$, then the left-hand bound above is satisfied with equality. In other words, the tightest upper bound on $P_{\mathrm{e}}\left(U_{2} \mid Y_{1}^{2} U_{1}\right)$ in terms of $P_{\mathrm{e}}\left(X_{1} \mid Y_{1}\right)$ only (i.e., independent of the particular distribution of $X_{1}$ and $Y_{1}$ ) is

$$
P_{\mathrm{e}}\left(U_{2} \mid Y_{1}^{2} U_{1}\right) \leq P_{\mathrm{e}}\left(X_{1} \mid Y_{1}\right) .
$$

Comparing this with (2.13) reveals the advantage of using the latter. 
We will prove Theorem 2.5 as a corollary to Lemma 2.9 and the following result.

Lemma 2.10. Let $B_{1}, B_{2}, \ldots$ be an i.i.d. process where $B_{1}$ is uniformly distributed over $\{-,+\}$. Also let $Z_{0}, Z_{1}, \ldots$ be a $[0,1]$-valued random process where $Z_{0}$ is constant and

$$
Z_{n} \leq \begin{cases}K Z_{n-1}, & \text { if } B_{n}=- \\ K Z_{n-1}^{2}, & \text { if } B_{n}=+\end{cases}
$$

for some finite $K>0$. Suppose also that $Z_{n}$ converges almost surely to a $\{0,1\}$-valued random variable $Z_{\infty}$ with $\operatorname{Pr}\left[Z_{\infty}=0\right]=z$. Then, for any $\beta<1 / 2$,

$$
\lim _{n \rightarrow \infty} \operatorname{Pr}\left[Z_{n} \leq 2^{-2^{n \beta}}\right]=z
$$

We defer the proof of Lemma 2.10 until Section 5, where we prove a more general result. We are now ready to prove Theorem 2.5:

Proof of Theorem 2.5. We will show that for all $\delta>0$ and sufficiently large $N$, the size of the set

$$
\mathcal{A}_{\beta}^{\prime \prime}:=\left\{i: Z\left(U_{i} \mid Y_{1}^{N} U_{1}^{i-1}\right) \leq 2^{-N^{\beta}}\right\}
$$

is at least $(1-H(X \mid Y)-\delta) N$, which will yield the lemma since the Bhattacharyya parameter upper bounds the average error probability. For this purpose, observe that the Bhattacharyya parameters obtained along the polarization construction satisfy the equalities

$$
\begin{aligned}
Z\left(U_{1} \mid Y_{1}^{N}\right) & =Z^{-\cdots--} \\
Z\left(U_{2} \mid Y_{1}^{N} U_{1}\right) & =Z^{-\cdots-+} \\
Z\left(U_{3} \mid Y_{1}^{N} U_{1}^{2}\right) & =Z^{-\cdots+-} \\
& \vdots \\
Z\left(U_{N-1} \mid Y_{1}^{N} U_{1}^{N-2}\right) & =Z^{+\cdots+-} \\
Z\left(U_{N} \mid Y_{1}^{N} U_{1}^{N-1}\right) & =Z^{+\cdots++},
\end{aligned}
$$


for any $N$. As in the proof of Theorem 2.3, define an i.i.d. process $B_{1}, B_{2}, \ldots$ with $\operatorname{Pr}\left[B_{1}=-\right]=\operatorname{Pr}\left[B_{1}=+\right]=1 / 2$, and a $[0,1]$-valued process $Z_{0}, Z_{1}, \ldots$ with

$$
\begin{aligned}
& Z_{0}=Z(X \mid Y) \\
& Z_{n}=Z_{n-1}^{B_{n}}, \quad n=1,2, \ldots
\end{aligned}
$$

Observe that $B_{1}, B_{2}, \ldots$ induces a uniform distribution on $Z_{n}$ over the set $\left\{Z^{-\cdots--}, \ldots, Z^{+\cdots++}\right\}$. Also, the almost sure convergence to $\{0,1\}$ of the process $H_{n}$, defined in (2.11) and Proposition 2.8 imply the almost sure convergence of $Z_{n}$ to the set $\{0,1\}$ with $\operatorname{Pr}\left[\lim _{n \rightarrow \infty} Z_{n}=0\right]$ $=1-H(X \mid Y)$. The claim then follows from Lemma 2.10 .

It is evident that the bounds in Lemma 2.9 are the only properties of the polarization construction that have a bearing upon the above proof. This brings out another technical appeal of polar codes: their large blocklength behavior can be inferred directly from the effect of the underlying one-step transformation on the Bhattacharyya parameters. This proves especially useful when one considers polar codes based on combining more than two random variables at a time. The recursive nature of such constructions ensure that the error probability behavior of the resulting codes can be analyzed with relative ease. We will discuss these constructions and their analysis in Section 5 .

\subsection{Polar Channel Coding}

In the previous section, we saw an entropy-achieving source coding scheme whose average error probability decays roughly exponentially in the square root of the blocklength. We will now see that the techniques we reviewed can be used, almost verbatim, to obtain capacity-achieving codes for binary-input symmetric memoryless channels.

Consider a binary-input discrete memoryless channel $W:\{0,1\} \rightarrow \mathcal{Y}$. Let $X_{1}, \ldots, X_{N}$ be a sequence of i.i.d. inputs to $N$ uses of $W$, and let $Y_{1}, \ldots, Y_{N}$ be the corresponding outputs (see Figure 2.4). Since the channel is memoryless and the inputs are i.i.d., the sequence $\left(X_{1}, Y_{1}\right), \ldots,\left(X_{N}, Y_{N}\right)$ is also i.i.d. This is exactly the same situation as in the previous sections, and one can imagine the following transmission scheme, which mimics the techniques we have seen: to 


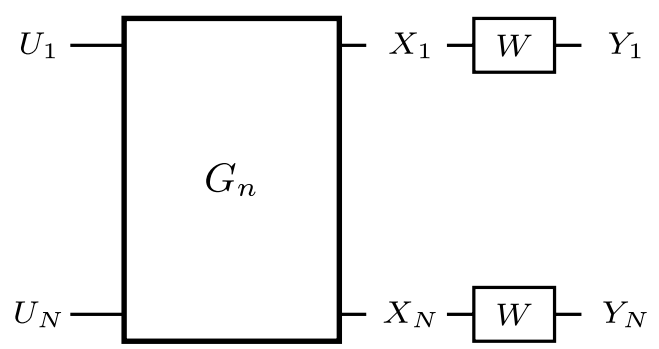

Fig. 2.4 Polar channel coding.

send the message corresponding to $X_{1}^{N}$, the encoder first computes $U_{1}^{N}=G_{n}\left(X_{1}^{N}\right)$ and reveals the bits with $P_{\mathrm{e}}\left(U_{i} \mid Y_{1}^{N} U_{1}^{i-1}\right) \geq 2^{-N^{\beta}}$ to the decoder, and sends $X_{1}^{N}$ through the channel. Upon receiving the channel output $Y_{1}^{N}$, the receiver decodes the unknown part of $U_{1}^{N}$ successively as in (2.6) and (2.7). It follows from Theorem 2.5 that the average block error probability of this coding scheme is $O\left(2^{-N^{\beta}}\right)$. Note that while all length- $N$ binary sequences are potential codewords in this scheme, a codeword chosen in an i.i.d. fashion will belong to the 'typical set' of size $\approx 2^{N H(X)}$ with high probability. Further, since approximately $N H(X \mid Y)$ bits of information are revealed to the receiver in advance, the effective rate of this code is approximately $I(X ; Y)$. Hence, by assigning the appropriate distribution to $X_{1}$, the capacity of the channel can be achieved.

The above coding argument is identical to the one in Section 2.3 but, while it is mathematically correct, it is inadequate from a channel coding perspective: First, observe that in the channel coding problem, the distribution on the channel inputs $X_{1}^{N}$ is induced by the encoder's choice of the distribution on $U_{1}^{N}$. This is in contrast with the source coding case, where the distribution of $X_{1}^{N}$ is intrinsic to the source, and the distribution of $U_{1}^{N}$ is induced by the transformation $G_{n}$. The difficulty is that in order to generate i.i.d. inputs $X_{1}^{N}$ to the channel, the encoder would have to choose $U_{1}^{N}$ from a non-uniform distribution, conflicting with the common assumption that the sender's messages are uniformly distributed. Second, in the source coding problem the values of the bits to be revealed to the receiver depend on the realization of the source $X_{1}^{N}$. In channel coding, however, these values need to be 
revealed to the receiver prior to communication, and therefore cannot depend on the particular message to be sent as proposed in the above scheme.

The first of these issues is of a somewhat technical nature, and can be dealt with most easily by insisting on uniformly distributed channel inputs $X_{1}^{N}$, since this would impose a uniform distribution on $U_{1}^{N}$. One can also circumvent the second issue by choosing the bits to be revealed in advance, and taking averages over the values of these bits. To make these arguments precise, let us consider the following coding scheme:

Code construction. Given a blocklength $N=2^{n}$, fix $0<\beta^{\prime}<\beta<1 / 2$ and find the set

$$
\mathcal{A}_{\beta}:=\left\{i: P_{\mathrm{e}}\left(U_{i} \mid Y_{1}^{N} U_{1}^{i-1}\right) \leq 2^{-N^{\beta}}\right\} .
$$

Choose $U_{i}, i \in \mathcal{A}_{\beta}^{c}$ independently and uniformly at random, and reveal their values to the receiver. The rate of the code will be $\left|\mathcal{A}_{\beta}\right| / N$.

Encoding. Given a uniformly distributed message $M \in\{0,1\}^{\left|\mathcal{A}_{\beta}\right|}$ to be transmitted, set $U_{\mathcal{A}_{\beta}}=M$. Transmit $X_{1}^{N}=G_{n}^{-1}\left(U_{1}^{N}\right)=G_{n}\left(U_{1}^{N}\right)$ over the channel.

Decoding. Upon receiving $Y_{1}^{N}$, the receiver decodes $U_{1}^{N}$ successively as in (2.6) and (2.7).

Rate and error probability. As $X_{1}^{N}$ is i.i.d. and uniformly distributed, we have $H(X)=1$, and therefore it follows from Theorem 2.5 that if $N$ is sufficiently large, the rate of the code is

$$
\left|\mathcal{A}_{\beta}\right| / N>1-H(X \mid Y)-\delta=I(X ; Y)-\delta .
$$

Note that $I(X ; Y)$ here is the symmetric capacity of the channel $W:\{0,1\} \rightarrow \mathcal{Y}$, the maximum rate achievable by binary codebooks with an equal fraction of zeros and ones. Note also that this is the true capacity for symmetric binary-input channels. It similarly follows from Theorem 2.5 and Proposition 2.1 that the block error probability of the above scheme, averaged over all messages and values of $U_{i}, i \in \mathcal{A}^{c}$, is $o\left(2^{-N^{\beta^{\prime}}}\right)$. Therefore there exists at least one set of values of bits $U_{i}, i \in \mathcal{A}^{c}$ - Arlkan calls these the frozen bits - for which the average block error probability of the resulting code is at most $o\left(2^{-N^{\beta^{\prime}}}\right)$. 
Turning this coding scheme into an explicit one requires one to fix the frozen bits to appropriate values. Recall that there is no such requirement for the source coding scheme of the previous section, since the unreliable bit values declared to the decoder are computed from the source realization, making the code explicit. For arbitrary binaryinput channels, finding the values of frozen bits that guarantee a low error probability is an open problem. However, we will now see that for the class of symmetric channels the above error probability bound holds irrespective of the values of the frozen bits and the message to be sent.

\subsubsection{Symmetric channels}

A binary-input discrete memoryless channel $W:\{0,1\} \rightarrow \mathcal{Y}$ is symmetric if there exists a permutation $\pi_{1}: \mathcal{Y} \rightarrow \mathcal{Y}$ such that $\pi_{1}^{-1}=\pi_{1}$ and $W(y \mid x)=W\left(\pi_{1}(y) \mid x+1\right)$. Following [4], we will let $\pi_{0}$ denote the identity permutation over $\mathcal{Y}$, and therefore we have $W\left(\pi_{a}(y) \mid\right.$ $x+a)=W(y \mid x)$ for $a=0,1$. We will use the more compact notation $W(i \cdot y \mid x):=W\left(\pi_{i}(y) \mid x\right)$.

We will now see that the upper bound the error probability of successive cancellation decoding is independent of $U_{1}^{N}$, and thus of the message to be sent and the frozen bit values. In order to do so, we will show that channels created by the polarization construction have certain symmetries. This will allow us to use the following simple property of symmetric channels: if the input to a symmetric channel is uniformly distributed, then the error probability of an optimal decoder - one that minimizes the average error probability - is independent of the channel input if the decoder makes a uniformly random decision whenever the output $y$ is such that $W(y \mid 0)=W(y \mid 1)$.

Observe that the error event of an optimal decoder for an arbitrary (i.e., possibly asymmetric) channel is contained in the event

$$
\left\{(x, y): p_{X \mid Y}(x \mid y) \leq p_{X \mid Y}(x+1 \mid y)\right\},
$$

which for uniform inputs is equivalent to the event

$$
\mathcal{B}:=\{(x, y): W(y \mid x) \leq W(y \mid x+1)\} .
$$


The probability of $\mathcal{B}$ is further upper bounded by the Bhattacharyya parameter of the channel, and thus we have

$$
P_{\mathrm{e}}(X \mid Y) \leq \operatorname{Pr}[\mathcal{B}] \leq Z(X \mid Y)
$$

These are in fact the same inequalities as in the proof of Proposition 2.7.

Recall that the $i$ th constituent of the successive cancellation decoder described in (2.6) and (2.7) is an optimal decoder for the channel $U_{i} \rightarrow$ $Y_{1}^{N} U_{1}^{i-1}$, although it breaks ties in favor of 1 rather than making a random decision. When the inputs $U_{1}^{N}$ are uniformly distributed, the likelihood ratio in (2.7) can be rewritten for arbitrary blocklength $N$ as

$$
L\left(y_{1}^{N}, u_{1}^{i-1}\right)=\frac{p_{Y_{1}^{N} U_{1}^{i-1} \mid U_{i}}\left(y_{1}^{N}, u_{1}^{i-1} \mid 0\right)}{p_{Y_{1}^{N} U_{1}^{i-1} \mid U_{i}}\left(y_{1}^{N}, u_{1}^{i-1} \mid 1\right)} .
$$

Clearly, the inequalities in the paragraph above also apply to polarized channels. Define the sets

$$
\mathcal{E}_{i}:=\left\{\left(u_{1}^{N}, y_{1}^{N}\right): p\left(y_{1}^{N}, u_{1}^{i-1} \mid u_{i}\right) \leq p\left(y_{1}^{N}, u_{1}^{i-1} \mid u_{i}+1\right)\right\} .
$$

We then have

$$
P_{\mathrm{e}}\left(U_{i} \mid Y_{1}^{N} U_{1}^{i-1}\right) \leq \operatorname{Pr}\left[\mathcal{E}_{i}\right] \leq Z\left(U_{i} \mid Y_{1}^{N} U_{1}^{i-1}\right)
$$

It is also clear that

$$
\operatorname{Pr}\left[\mathcal{E}_{i} \mid U_{1}^{N}=u_{1}^{N}\right]:=\operatorname{Pr}\left[\left(U_{1}^{N}, Y_{1}^{N}\right) \in \mathcal{E}_{i} \mid U_{1}^{N}=u_{1}^{N}\right]
$$

upper bounds the error probability of decoding $u_{i}$ when $u_{1}^{N}$ is sent. We will show that the above probability is the same for all $u_{1}^{N}$, that is, $\operatorname{Pr}\left[\mathcal{E}_{i}\right]=\operatorname{Pr}\left[\mathcal{E}_{i} \mid U_{1}^{N}=u_{1}^{N}\right]$. Since the block error probability of successive cancellation was upper bounded, through Propositions 2.1 and 2.7 , by

$$
\sum_{i \in \mathcal{A}} Z\left(U_{i} \mid Y_{1}^{N} U_{1}^{i-1}\right)
$$

it will follow from (2.15) that the same upper bound holds irrespective of the value of $U_{1}^{N}$. 
The main ingredient of the proof is the symmetry in the created channels:

Proposition 2.11. If $W:\{0,1\} \rightarrow \mathcal{Y}$ is symmetric, then the channels $p\left(y_{1}^{N} \mid u_{1}^{N}\right)$ and $p\left(y_{1}^{N}, u_{1}^{i-1} \mid u_{i}\right)$ have the following symmetries:

$$
\begin{aligned}
p\left(y_{1}^{N} \mid u_{1}^{N}\right) & =p\left(G_{n}\left(a_{1}^{N}\right) \cdot y_{1}^{N} \mid u_{1}^{N}+a_{1}^{N}\right) \\
p\left(y_{1}^{N}, u_{1}^{i-1} \mid u_{i}\right) & =p\left(G_{n}\left(a_{1}^{N}\right) \cdot y_{1}^{N}, u_{1}^{i-1}+a_{1}^{i-1} \mid u_{i}+a_{i}\right)
\end{aligned}
$$

for all $a_{1}^{N} \in\{0,1\}^{N}$.

Proof. The proof follows from the linearity of $G_{n}$, and thus the result is valid for any linear transform. Set $x_{1}^{N}=G_{n}\left(u_{1}^{N}\right)$ and $b_{1}^{N}=G_{n}\left(a_{1}^{N}\right)$. The first claim is obtained through the following inequalities:

$$
\begin{aligned}
p\left(b_{1}^{N} \cdot y_{1}^{N} \mid u_{1}^{N}+a_{1}^{N}\right) & =W^{N}\left(b_{1}^{N} \cdot y_{1}^{N} \mid x_{1}^{N}+b_{1}^{N}\right) \\
& =W^{N}\left(b_{1}^{N} \cdot b_{1}^{N} \cdot y_{1}^{N} \mid x_{1}^{N}\right) \\
& =W^{N}\left(y_{1}^{N} \mid x_{1}^{N}\right) \\
& =p\left(y_{1}^{N} \mid u_{1}^{N}\right)
\end{aligned}
$$

Here the first equality follows from the linearity of $G_{n}$, the second from $W$ being symmetric, and the third from the fact that $\pi_{0}=\pi_{0}^{-1}$ and $\pi_{1}=\pi_{1}^{-1}$. To obtain the second claim, we write

$$
\begin{aligned}
p\left(y_{1}^{N}, u_{1}^{i-1} \mid u_{i}\right) & =\frac{1}{2^{N-1}} \sum_{u_{i+1}^{N}} p\left(y_{1}^{N} \mid u_{1}^{N}\right) \\
& =\frac{1}{2^{N-1}} \sum_{u_{i+1}^{N}} p\left(b_{1}^{N} \cdot y_{1}^{N} \mid u_{1}^{N}+a_{1}^{N}\right) \\
& =p\left(b_{1}^{N} \cdot y_{1}^{N}, u_{1}^{i-1}+a_{1}^{i-1} \mid u_{i}+a_{i}\right),
\end{aligned}
$$

where the second equality follows from the first claim, and the third equality is obtained by observing that the summation over $u_{i+1}^{N}$ is equivalent to a summation over $u_{i+1}^{N}+a_{i+1}^{N}$.

We are now ready to prove the claimed result. 
Proposition 2.12. $\operatorname{Pr}\left[\mathcal{E}_{i} \mid U_{1}^{N}=u_{1}^{N}\right]=\operatorname{Pr}\left[\mathcal{E}_{i}\right]$ for all $i$ and $u_{1}^{N} \in$ $\{0,1\}^{N}$.

Proof. First note that the symmetry in the channel $p\left(y_{1}^{N}, u_{1}^{i-1} \mid u_{i}\right)$ and the definition of $\mathcal{E}_{i}$ imply

$$
\left(u_{1}^{N}, y_{1}^{N}\right) \in \mathcal{E}_{i} \text { if and only if }\left(u_{1}^{N}+a_{1}^{N}, G_{n}\left(a_{1}^{N}\right) \cdot y_{1}^{N}\right) \in \mathcal{E}_{i} .
$$

We can then write

$$
\begin{aligned}
\operatorname{Pr}\left[\mathcal{E}_{i} \mid U_{1}^{N}=u_{1}^{N}\right] & =\sum_{y_{1}^{N}} p\left(y_{1}^{N} \mid u_{1}^{N}\right) \mathbb{1}_{\left[\left(u_{1}^{N}, y_{1}^{N}\right) \in \mathcal{E}_{i}\right]} \\
& =\sum_{y_{1}^{N}} p\left(x_{1}^{N} \cdot y_{1}^{N} \mid 0^{N}\right) \mathbb{1}_{\left.\left[\left(0^{N}, x_{1}^{N} \cdot y_{1}^{N}\right) \in \mathcal{E}_{i}\right)\right]} \\
& =\operatorname{Pr}\left[\mathcal{E}_{i} \mid U_{1}^{N}=0^{N}\right] .
\end{aligned}
$$

The last equality above is obtained by observing that the sum over $y_{1}^{N}$ is equivalent to a sum over $x_{1}^{N} \cdot y_{1}^{N}$. This yields the claim.

\subsection{Performance}

As we saw in Corollary 2.6, the error probability of polar codes decay roughly exponentially in the square root of the blocklength. Unfortunately, this performance guarantee is an asymptotic one, and the proof of Lemma 2.10 in Section 4 suggests that one may need to take very large blocklengths to observe the promised decay. Here we will see corroborating evidence to this prediction: at short blocklengths, polar codes' performance under successive cancellation decoding is inferior to that of state-of-the-art codes (Figure 2.5). This disadvantage seems to be largely due to the successive cancellation decoder, and there has been successful attempts to eliminate it. In [44], Tal and Vardy observed that the error performance can be improved if one avoids making hard decisions on the bit values at each decoding step. Instead, the authors proposed a successive cancellation 'list decoder', which in each step maintains a small list containing the most likely values for the most recent bits. The complexity of this decoder is within a factor of the 
list size from the original decoder's - we will see in Section 3 that the latter complexity is $O(N \log N)$ - and is thus a reasonable alternative for small list sizes. The error probability improvement attained by this decoder can be seen in Figure 2.5. Even more dramatic gains are possible through two simple modifications. The first of these is to allow the list decoder to produce a list of candidate codewords at the final decoding step (as opposed to a single codeword), and to use an external method - e.g., a cyclic redundancy check as in [44] to eliminate incompatible candidates. The second is to make the code systematic, i.e., to have the information bits appear as part of the transmitted codeword, as Arıkan shows in [7]. Although this does not affect the block error probability, empirical evidence shows that the bit error probability can be improved through this modification. See Figure 2.5 for a performance comparison of these schemes.

\section{A Proof of Lemma 2.2}

Let $R_{1}$ and $R_{2}$ be $[0,1 / 2]$-valued random variables defined through

$$
\begin{aligned}
& R_{1}=\min \left\{p_{X_{1} \mid Y_{1}}\left(0 \mid y_{1}\right), p_{X_{1} \mid Y_{1}}\left(1 \mid y_{1}\right)\right\} \text { whenever } Y_{1}=y_{1}, \\
& R_{2}=\min \left\{p_{X_{2} \mid Y_{2}}\left(0 \mid y_{2}\right), p_{X_{2} \mid Y_{2}}\left(1 \mid y_{2}\right)\right\} \text { whenever } Y_{2}=y_{2} .
\end{aligned}
$$

For $a, b \in[0,1]$ define

$$
a * b=a(1-b)+(1-a) b .
$$

Also let $h:[0,1 / 2] \rightarrow[0,1]$ denote the binary entropy function. With these definitions, we have

$$
H\left(X_{1}+X_{2} \mid Y_{1}^{2}\right)=E\left[h\left(R_{1} * R_{2}\right)\right]
$$

Both claims of the lemma follow from the convexity of the function $h\left(a * h^{-1}(t)\right)$ in $t \in[0,1 / 2]$, which was established in [48]. In particular, we have

$$
\begin{aligned}
H\left(X_{1}+X_{2} \mid Y_{1}^{2}\right) & =E\left[h\left(R_{1} * R_{2}\right)\right] \\
& =E\left[E\left[h\left(R_{1} * R_{2}\right)\right] \mid R_{1}\right] \\
& =E\left[E\left[h\left(R_{1} * h^{-1}\left(h\left(R_{2}\right)\right)\right)\right] \mid R_{1}\right] \\
& \geq E\left[h\left(R_{1} * h^{-1}\left(E\left[h\left(R_{2}\right)\right]\right)\right)\right] \\
& =E\left[h\left(R_{1} * h^{-1}(\beta)\right)\right] .
\end{aligned}
$$




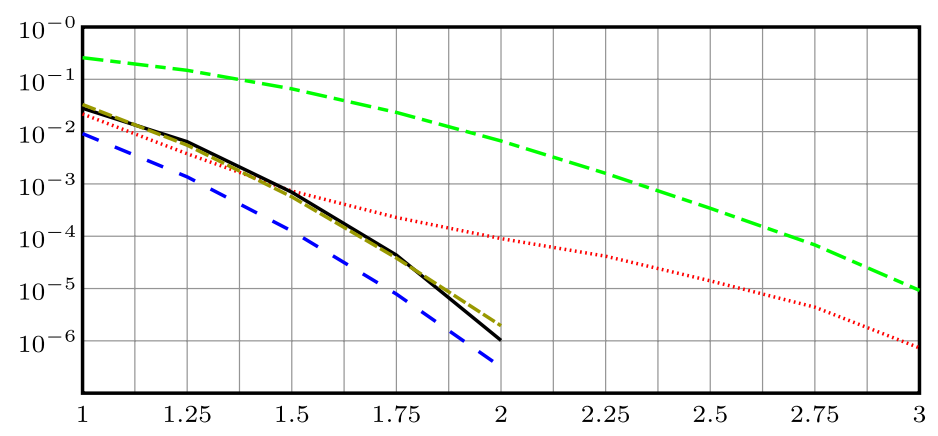

$----------------------------$

Polar code of rate $1 / 2$, length 2048 , with successive cancellation decoding.

Polar code of rate $1 / 2$, length 2048 , with successive cancellation list decoding (list size $=32$ ).

LDPC code of rate $1 / 2$, length 2304, with 32 iterations of belief propagation decoder, as implemented in the WiMAX standard.

Polar code of rate $1 / 2$, length 2048 , with successive cancellation list decoder and 16-bit CRC.

Systematic polar code of rate $1 / 2, \overline{2}$, length 2048 , with successive cancellation list decoder and 16-bit CRC.

Fig. 2.5 Bit error probability comparison for transmission over a binary-input additive white Gaussian noise channel. Horizontal axis shows the signal-to-noise ratio. Polar codes are optimized for this channel using a variation of the Tal-Vardy algorithm we will see in Section 3. Plots are due to E. Arıkan, W. Gross, I. Tal, and A. Vardy.

Applying the convexity of $h\left(a * h^{-1}(t)\right)$ a second time we obtain

$$
\begin{aligned}
H\left(X_{1}+X_{2} \mid Y_{1}^{2}\right) & \geq E\left[h\left(R_{1} * h^{-1}(\beta)\right)\right] \\
& =E\left[h\left(h^{-1}\left(h\left(R_{1}\right)\right) * h^{-1}(\beta)\right)\right] \\
& \geq h\left(h^{-1}\left(E\left[h\left(R_{1}\right)\right]\right) * h^{-1}(\beta)\right) \\
& =h\left(h^{-1}(\alpha) * h^{-1}(\beta)\right) .
\end{aligned}
$$


It is easy to see that the last term is the equal to $H\left(X_{1}+X_{2} \mid Y_{1}^{2}\right)$ when $\left(X_{1}, Y_{1}\right)$ and $\left(X_{2}, Y_{2}\right)$ are distributed as in (i), yielding the claim. To see the second claim, note that the convexity of $h\left(a * h^{-1}(t)\right)$ implies

$$
\begin{aligned}
h\left(a * h^{-1}(t)\right) & \leq t h\left(a * h^{-1}(1)\right)+(1-t) h\left(a * h^{-1}(0)\right) \\
& =t+(1-t) h(a) .
\end{aligned}
$$

It then follows that

$$
\begin{aligned}
H\left(X_{1}+X_{2} \mid Y_{1}^{2}\right) & =E\left[h\left(R_{1} * R_{2}\right)\right] \\
& =E\left[h\left(R_{1} * h^{-1}\left(h\left(R_{2}\right)\right)\right)\right] \\
& \leq E\left[h\left(R_{1}\right)+h\left(R_{2}\right)-h\left(R_{1}\right) h\left(R_{2}\right)\right] \\
& =E\left[h\left(R_{1}\right)\right]+E\left[h\left(R_{2}\right)\right]-E\left[h\left(R_{1}\right)\right] E\left[h\left(R_{2}\right)\right] .
\end{aligned}
$$

where the last equality follows from the independence between $R_{1}$ and $R_{2}$. A simple calculation shows that the last term is equal to $H\left(X_{1}+X_{2} \mid Y_{1}^{2}\right)$ when $\left(X_{1}, Y_{1}\right)$ and $\left(X_{2}, Y_{2}\right)$ are distributed as in (ii), completing the proof. 


\section{Complexity}

We saw in the previous section that recursively applying a certain two-by-two transform to a memoryless binary source or channel leads to polarization, yielding source and channel codes that achieve optimal rates. It is clear from these coding schemes that there are three problems of complexity that need to be addressed: (i) complexity of encoding, i.e., computing the function $G_{n}$, (ii) complexity of decoding, i.e., computing the probabilities appearing in Equation (2.6), and (iii) complexity of construction, i.e., determining the set of bit indices with small error probabilities. Thanks to the recursive nature of the construction, all three tasks can be broken down to similar tasks of smaller sizes. We will see that one can take advantage of this fact to accomplish these tasks with low time and space complexities. Throughout this section, time complexities will be given for a single-processor machine with random-access memory, on which a single infinite-precision arithmetic operation takes unit time.

\subsection{Encoding}

Recall from Section 2 that encoding in polar source coding amounts to computing $X_{1}^{N}=G_{n}\left(U_{1}^{N}\right)$ from the source vector $U_{1}^{N}$, where $G_{n}$ is 
defined recursively through

$$
\begin{aligned}
G_{0}(u) & =u, \\
G_{n}\left(u_{1}, u_{2}\right) & =\pi_{n}\left(G_{n-1}\left(u_{1}\right)+G_{n-1}\left(u_{2}\right), G_{n-1}\left(u_{2}\right)\right), \quad n=1,2, \ldots
\end{aligned}
$$

where $u_{1}, u_{2} \in\{0,1\}^{N / 2}$. In polar channel coding, on the other hand, encoding consists in performing the mapping $G_{n}^{-1}$. These tasks are equivalent. It is indeed not difficult to show that $G_{n}^{-1}=G_{n}$. (A proof of this can be found in [4].)

Let $\mathcal{K}_{N}$ denote the time complexity of computing $G_{n}$. Assuming that permuting $N$ elements takes $N$ units of time, and that binary addition takes unit time, it is clear from the definition of $G_{n}$ that $\mathcal{K}_{N} \leq 2 \mathcal{K}_{N / 2}+N / 2+N$. If one takes $\mathcal{K}_{1}=1$, it can then be shown by induction that

$$
\mathcal{K}_{N} \leq \frac{3}{2} N \log N+N
$$

i.e., the time complexity of encoding is $O(N \log N)$. An implementation of polar encoding is depicted in Figure 3.1. It is clear that at each stage of computation only the $N$ incoming bit values from the previous stage need to be remembered. Therefore the space complexity of encoding is $O(N)$.

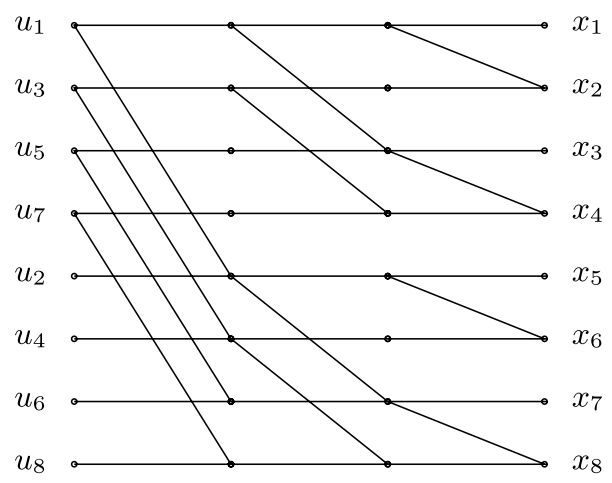

Fig. 3.1 An implementation of polar source encoding for $n=3(N=8)$. Computation is performed in three stages, from left to right. In each, nodes compute the modulo- 2 sum of the incoming bit values on the right and send the result through the edges on the left. The order of computation is reversed in channel encoding. 


\subsection{Decoding}

Recall the successive decoding rule given in Section 2:

$$
\hat{u}_{i}= \begin{cases}u_{i}, & \text { if } i \in \mathcal{A}^{c} \\ 0, & \text { if } i \in \mathcal{A} \text { and } L\left(y_{1}^{N}, \hat{u}_{1}^{i-1}\right)>1 \\ 1, & \text { otherwise }\end{cases}
$$

where

$$
L\left(y_{1}^{N}, \hat{u}_{1}^{i-1}\right)=\frac{p_{U_{i} \mid Y_{1}^{N} U_{1}^{i-1}}\left(0 \mid y_{1}^{N}, \hat{u}_{1}^{i-1}\right)}{p_{U_{i} \mid Y_{1}^{N} U_{1}^{i-1}}\left(1 \mid y_{1}^{N}, \hat{u}_{1}^{i-1}\right)} .
$$

We will see that the complexity of this decoder is $O(N \log N)$. To simplify the analysis, we will estimate the total complexity of producing $\hat{u}_{1}^{N}$ followed by computing $\hat{x}_{1}^{N}=G_{n}\left(\hat{u}_{1}^{N}\right)$ from it.

Given distribution $p:=p_{X Y}$, let $p^{-}$and $p^{+}$denote its descendant distributions

$$
\begin{aligned}
& p^{-}:=p_{U_{1}\left(Y_{1}^{2}\right)} \\
& p^{+}:=p_{U_{2}\left(Y_{1}^{2} U_{1}\right)} .
\end{aligned}
$$

Note that the increasing order of decoded bits $1, \ldots, N$ in (3.1) corresponds to the order $p^{-\ldots--}, p^{-\ldots-+}, \ldots, p^{+\ldots+}$ in the underlying distributions. Therefore the task of decoding the $N$ bits that descend from $p$ can be decomposed into two similar tasks of smaller size: decoding bits $\hat{u}_{1}^{N / 2}$, all of which descend from $p^{-}$, followed by decoding bits $\hat{u}_{N / 2+1}^{N}$, all of which descend from $p^{+}$. This can be accomplished as follows: Upon observing $y_{1}^{N}$, we initialize the first decoder by computing the conditional distributions

$$
p_{i}^{-}\left(x \mid y_{2 i-1}^{2 i}\right):=\sum_{u \in \mathcal{X}} p_{X \mid Y}\left(x+u \mid y_{2 i-1}\right) p_{X \mid Y}\left(u \mid y_{2 i}\right), \quad x=0,1,
$$

$i=1, \ldots, N / 2$. The decoder uses these to estimate $\hat{u}_{1}^{N / 2}$ and passes $s_{1}^{N / 2}=G_{n-1}\left(\hat{u}_{1}^{N / 2}\right)$ to the second decoder, which is then initialized by computing the conditional distributions

$$
\begin{aligned}
& p_{i}^{+}\left(x \mid y_{2 i-1}^{2 i}, s_{i}\right):=p_{X \mid Y}\left(x+s_{i} \mid y_{2 i-1}\right) p_{X \mid Y}\left(x \mid y_{2 i}\right) / p_{i}^{-}\left(x_{i} \mid y_{2 i-1}^{2 i}\right) . \\
& i=1, \ldots, N / 2 \text { to decode } \hat{u}_{N / 2+1}^{N} \text {. The decoder then produces } t_{1}^{N / 2}:= \\
& G_{n-1}\left(\hat{u}_{N / 2+1}^{N}\right) \text {. The estimate } \hat{x}_{1}^{N} \text { is then computed as } \hat{x}_{1}^{N}=\left(s_{1}^{N / 2}+\right. \\
& \left.t_{1}^{N / 2}, t_{1}^{N / 2}\right) .
\end{aligned}
$$


The recursive nature of $G_{n}$ implies that the tasks of the two decoders can similarly be decomposed into smaller tasks. This decomposition can be continued $n$ times until one obtains $N$ decoding tasks of blocklength one. Let $\chi_{N}$ denote the total time complexity of producing $\hat{u}_{1}^{N}$ and computing $\hat{x}_{1}^{N}=G_{n}\left(\hat{u}_{1}^{N}\right)$ from the decoded word. As we saw in the above description, this task at blocklength $N$ involves two similar tasks at length $N / 2$. The additional tasks of computing the $N$ conditional distributions to initialize the two decoders at length $N / 2$, passing the output of the first decoder to the second, and assembling the results to generate $\hat{x}_{1}^{N}$ require $\alpha N$ operations for some constant $\alpha$. Therefore we have,

$$
\chi_{N} \leq 2 \chi_{N / 2}+\alpha N
$$

Applying this bound recursively we see that $\chi_{N} \leq N+\alpha N \log N$, i.e., that successive cancellation decoding can be performed in time $O(N \log N)$. By similar arguments it can be seen that the space complexity of the decoder at length $N$ can also be bounded as in (3.3). Therefore the overall space complexity is also $O(N \log N)$.

Note that the above complexity figures are given for a single processor machine. If one has multiple processors and carefully schedules the intermediate decoding operations, then both the time and the space complexities can be reduced to $O(N)$. We refer the reader to [30] for details.

\subsection{Construction}

Recall the main premise of polar source and channel coding: reliable bits obtained through a polarizing transform can be decoded with small error probability as long as the values of the unreliable bits are provided in advance to the decoder. In Section 2, a source code of rate $k / N$ and blocklength $N$ was chosen by revealing to the receiver the values of $U_{i}$ for which $Z\left(U_{i} \mid Y_{1}^{N} U_{1}^{i-1}\right)$ are among the $k$ largest. In polar channel coding, the same method yields a code of rate $1-k / N$. Note that this is not the only reasonable definition of the set of unreliable bits; one could for instance replace $Z\left(U_{i} \mid Y_{1}^{N} U_{1}^{i-1}\right)$ with $H\left(U_{i} \mid Y_{1}^{N} U_{1}^{i-1}\right)$ or $P_{\mathrm{e}}\left(U_{i} \mid Y_{1}^{N} U_{1}^{i-1}\right)$ as the figure of merit in the above scheme and still expect the resulting code to perform well. 
In order to construct a polar code, one may compute all $N$ Bhattacharyya parameters

$$
Z\left(U_{i} \mid Y_{1}^{N} U_{1}^{i-1}\right)=2 \sum_{y_{1}^{N}, u_{1}^{i-1}} \sqrt{p\left(0, y_{1}^{N} u_{1}^{i-1}\right) p\left(1, y_{1}^{N} u_{1}^{i-1}\right)}
$$

and sort them to determine the $k$ largest. It can easily be shown that the probability terms on the right-hand-side can be computed in time $O(N \log N)$ for fixed $y_{1}^{N}, u_{1}^{i-1}$. However, there are $|\mathcal{Y}|^{N} \cdot 2^{i-1}$ terms in the summation, and no sub-exponential-time (in $N$ ) algorithm to compute it exactly is known. (One exception to this is the case where the channel between the $X$ and $Y$ is a binary erasure channel. See Section 3.3.1.) Clearly, the difficulty here is the linear growth of the number of random variables $Y_{1}^{N}, U_{1}^{i-1}$ in $N$.

One way to circumvent this issue is to quantize the alphabets $\mathcal{Y}^{N} \times\{0,1\}^{i-1}$ to smaller ones. In order to be of practical relevance, a quantization method must have low computational complexity and approximate the Bhattacharyya parameters (or any other meaningful figure of merit) closely. The algorithm we will now see was given in [45] and satisfies both of these requirements. The analysis offered here is based on [36].

Let $(X, Y, T)$ be random variables such that $X \in\{0,1\}$ and $X-Y-T$ is a Markov chain. Following the definition of channel degradation, we will say that the distribution $p_{X Y}$ is (physically) degraded with respect to $p_{X T} \cdot{ }^{1}$ Clearly, we have

$$
H(X \mid Y) \leq H(X \mid T) \text { and } Z(X \mid Y) \leq Z(X \mid T) .
$$

It also follows that applying the polarization transform to $X$ (see Figure 3.2) yields the Markov chains

$$
U_{1}-Y_{1}^{2}-T_{1}^{2} \text { and } \quad U_{2}-Y_{1}^{2} U_{1}-T_{1}^{2} U_{1} .
$$

That is, letting $p_{X Y}^{-}=p_{U_{1}\left(Y_{1}^{2}\right)}$ and $p_{X Y}^{+}=p_{U_{2}\left(Y_{1}^{2} U_{1}\right)}$, we see that degradation between $p_{X Y}$ and $p_{X T}$ is preserved between their polarized versions: $p_{X T}^{-}$(respectively, $p_{X T}^{+}$) is degraded with respect to

${ }^{1}$ Physical degradation is assumed here for simplicity. The statements that follow are also valid under stochastic degradation. 


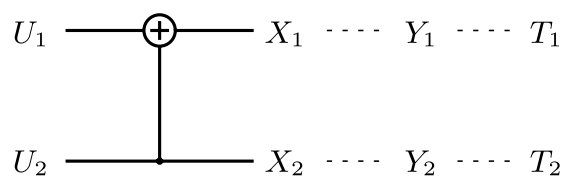

Fig. 3.2 Degradation between $p_{X Y}$ and $p_{X T}$ is preserved between their polarized descendants.

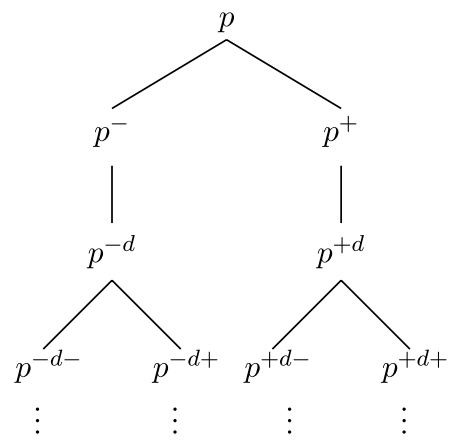

Fig. 3.3 Approximate distributions obtained through the procedure described in Steps 0-3. Each distribution on the tree is degraded with respect to its original counterpart. (For instance, $p^{-d+}$ is degraded with respect to $p^{-+}$.)

$p_{X Y}^{-}$(respectively, $p_{X Y}^{+}$). It follows immediately that degradation is preserved between all polarized descendants of $p_{X Y}$ and $p_{X T}$, and thus we have

$$
\begin{aligned}
H\left(U_{i} \mid Y_{1}^{N} U_{1}^{i-1}\right) & \leq H\left(U_{i} \mid T_{1}^{N} U_{1}^{i-1}\right), \\
Z\left(U_{i} \mid Y_{1}^{N} U_{1}^{i-1}\right) & \leq Z\left(U_{i} \mid T_{1}^{N} U_{1}^{i-1}\right) .
\end{aligned}
$$

The approximation algorithm we will see replaces polarized descendants of $p_{X Y}$ with degraded versions that are much simpler to describe. It consists of the following steps (see Figure 3.3):

Step 0: Fix an integer $L$. Set $S=\left\{p_{X Y}\right\}$.

Step 1: Update $S$ by replacing each $p \in S$ with one-step polarized versions $p^{-}$and $p^{+}$.

Step 2: Update $S$ by replacing each $p \in S$ with a degraded version $p^{d}$ whose alphabet size is no larger than $2 L$.

Step 3: Go to Step 1.

It is readily seen that the procedure above is identical to the polarization construction except for the additional Step 2, where the 
distributions obtained at each polarization stage are replaced with simpler ones in order to curb the growth in the alphabet sizes. At the end of the procedure, all distributions have an alphabet size of at most $2 L$, and thus their Bhattacharyya parameters can easily be computed for moderate values of $L$. One can then construct a code based on these values. Since degradation is a transitive relation, distributions obtained through the procedure above are degraded with respect to their original counterparts (Figure 3.3). That is, this procedure overestimates the Bhattacharyya parameters, and thus produces a subset of the true reliable bits.

Clearly, the number of true reliable bits 'missed' by the procedure (i.e., the rate loss) will depend on the bound $L$ on the alphabet size, and on how degrading is performed in Step 2. For a given $L$, one may attempt to find the degrading operation that minimizes the number of unidentified reliable bits, but this appears to be a difficult task. A seemingly simpler task is to minimize the average increase in the Bhattacharyya parameters at each recursion, but this too turns out to be an analytically and computationally difficult problem. Nevertheless, there exist suboptimal but efficient degrading methods that yield reasonably small rate losses even for modest values of $L$, as we will see next.

To degrade distributions, we will think of $X \in\{0,1\}$ as the input to the channel $p_{Y \mid X}$. We will concatenate the channel with one that merges output symbols $y \in \mathcal{Y}$ that induce similar conditional distributions on the input. Set the shorthand notation $p_{y}:=p_{X \mid Y=y}$. Partition $\mathcal{Y}$ into sets $\mathcal{Y}_{i, j}, i=1, \ldots, L, j=0,1$ and $\mathcal{Y}_{L+1}$ defined through

$$
\begin{aligned}
\mathcal{Y}_{i, j} & =\left\{y: p_{y}(j)>p_{y}(j+1), \frac{i-1}{L} \leq H\left(p_{y}\right)<\frac{i}{L}\right\}, \quad i=1, \ldots, L, \\
\mathcal{Y}_{L+1} & =\left\{y: p_{y}(0)=p_{y}(1)\right\} .
\end{aligned}
$$

Here, $H\left(p_{y}\right)$ denotes the entropy of the distribution $p_{y}$. Now let $T$ be a random variable taking values in $\{1, \ldots, L\} \times\{0,1\} \cup\{L+1\}$ such that $X-Y-T$ is a Markov chain, where for all $y$ with $p(y)>0$ we have

$$
p_{T \mid Y}(t \mid y)=\left\{\begin{array}{ll}
1, & \text { if } y \in \mathcal{Y}_{t} \\
0, & \text { otherwise }
\end{array} .\right.
$$

Note that $T$ can be the single symbol $L+1$ or a pair of symbols $(i, j)$. Also observe that the channel $p_{T \mid Y}$ merges symbols $y \in \mathcal{Y}$ for which 


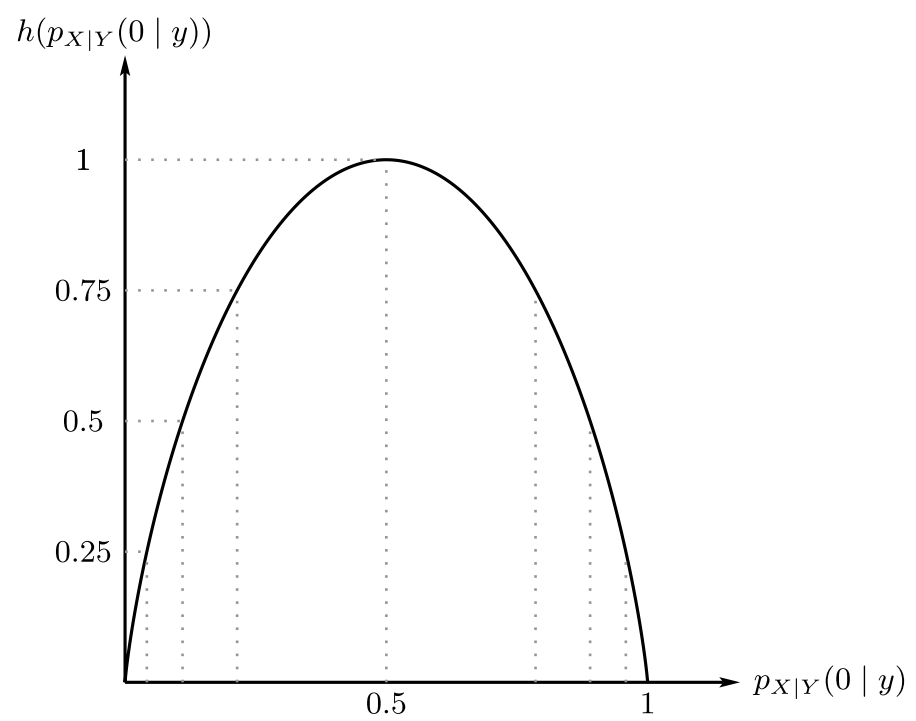

Fig. 3.4 Each output symbol $y$ is placed in one of the horizontal bins based on the value of $p_{X \mid Y}(0 \mid y)$. In this figure, $L=4$.

the conditional input distributions $p_{y}$ and their entropies $H\left(p_{y}\right)$ are close (see Figure 3.4). We can now complete the description of the approximation algorithm on page 297 by specifying Step 2:

Step 2: Replace each distribution $p_{X Y}$ from Step 1 with $p_{X T}$ obtained through (3.5).

We will first show that the information loss incurred at Step 2 is small, in the sense that $H(X \mid T)-H(X \mid Y) \leq 1 / L$. To see this, observe that the difference can be written as

$$
\begin{aligned}
& H(X \mid T)-H(X \mid Y) \\
& \quad=\sum_{t} p(t) H(X \mid T=t)-\sum_{y} p(y) H(X \mid Y=y) \\
& \quad=\sum_{t}\left[p(t) H(X \mid T=t)-\sum_{y \in \mathcal{Y}_{t}} p(y) H(X \mid Y=y)\right] \\
& \quad=\sum_{t} \sum_{y \in \mathcal{Y}_{t}} p(y)[H(X \mid T=t)-H(X \mid Y=y)],
\end{aligned}
$$


where we used the relation

$$
p(t)=\sum_{y \in \mathcal{Y}_{t}} p(y)
$$

to obtain the third equality. Observe that the entropy difference above is zero for $t=L$. It is also easily seen that $p(x \mid t)$ is a convex combination of $p(x \mid y), y \in \mathcal{Y}_{t}$, that is,

$$
p(x \mid t)=\sum_{y \in \mathcal{Y}_{t}} \frac{p(y)}{p(t)} p(x \mid y) .
$$

Since the binary entropy function is monotonic in the intervals $[0,1 / 2]$ and $[1 / 2,1]$, it follows from (3.4) and (3.7) that for all $t=(i, j)$

$$
p_{X \mid T}(j \mid t)>p_{X \mid T}(j+1 \mid t) \quad \text { and } \quad \frac{i-1}{L} \leq H(X \mid T=t)<\frac{i}{L} .
$$

That is, each entropy difference $H(X \mid T=t)-H(X \mid Y=y)$ in the above summation is upper bounded by $1 / L$, and thus

$$
H(X \mid T)-H(X \mid Y) \leq 1 / L .
$$

We will use this result to bound the overall loss after several recursions of the approximation algorithm. For this purpose, let $H,\left(H^{-}, H^{+}\right),\left(H^{--}, H^{-+}, H^{+-}, H^{++}\right), \ldots$ denote the polarized entropies as before. Also let $H^{-d}$ (respectively, $H^{+d}$ ) denote the entropy obtained from $H$ by a '-' (respectively, ' + ') operation followed by degradation. Similarly let $H^{s_{1} d \ldots s_{n} d}, s_{1}^{n} \in\{-,+\}^{n}$ denote the entropies obtained after $n$ recursions of the approximation algorithm. We will bound the total gap

$$
\sum_{s_{1}^{n} \in\{-,+\}^{n}} H^{s_{1} d \ldots s_{n} d}-H^{s_{1} \ldots s_{n}}=\sum_{s_{1}^{n} \in\{-,+\}^{n}} H^{s_{1} d \ldots s_{n} d}-2^{n} H
$$

between the original entropies and their estimates. In order to do so, we will first bound the loss incurred at the $n$th recursion of the algorithm using (3.8):

$$
\begin{aligned}
& H^{s_{1} d \ldots s_{n-1} d-d}+H^{s_{1} d \ldots s_{n-1} d+d} \\
& \quad \leq H^{s_{1} d \ldots s_{n-1} d-}+H^{s_{1} d \ldots s_{n-1} d+}+2 / L \\
& \quad=2\left(H^{s_{1} d \ldots s_{n-1} d}+1 / L\right) .
\end{aligned}
$$


Summing over $s_{1}, \ldots, s_{n}$ we obtain

$$
\sum_{s_{1}^{n}} H^{s_{1} d \ldots s_{n} d} \leq 2 \sum_{s_{1}^{n-1}} H^{s_{1} d \ldots s_{n-1} d}+2^{n} / L .
$$

Now observe that we can apply inequality (3.9) to the right-hand-side of the above to bound the loss in the $(n-1)$ th recursion. Doing this repeatedly for each recursion of the algorithm we obtain

$$
\sum_{s_{1}^{n}} H^{s_{1} d \ldots s_{n} d} \leq 2^{n} H+n 2^{n} / L,
$$

or equivalently

$$
\frac{1}{2^{n}} \sum_{s_{1}^{n}} H^{s_{1} d \ldots s_{n} d}-H^{s_{1} \ldots s_{n}} \leq n / L,
$$

All of the $2^{n}$ differences in the above sum are non-negative, from which it follows that at least a $1-\sqrt{n / L}$ fraction of the estimated entropies $H^{s_{1} d \ldots s_{n} d}$ are at most $\sqrt{n / L}$ larger than the true values $H^{s_{1} \ldots s_{n}}$. Hence, by choosing $L$ large - say $L=n^{2}$ — one can estimate a significant fraction of the entropies closely. Since the true entropies polarize to 0 and 1 , by choosing a sufficiently large $n$ and $L=n^{2}$, one can identify at least $(1-\delta) H N$ bit indices with entropies and Bhattacharyya parameters at most $\delta$, for any given $\delta>0$.

The analysis thus far is inadequate from an error probability standpoint. In fact, continuing the above statements with an application of the union bound only guarantees a block error probability $\delta(1-\delta) H N$, although we saw in Corollary 2.6 that the block error probability of polar codes is roughly $O\left(2^{-\sqrt{N}}\right)$. Fortunately, this shortcoming can be remedied by the following augmentation to the algorithm:

i. At the $n$th recursion, replace all good approximate distributions, i.e., those with Bhattacharyya parameters less than $\delta$, with erasure distributions (defined in Section 3.3.1) with the same Bhattacharyya parameter.

ii. Polarize only these distributions $m$ more times without degradation. 
We will see below that the descendants of erasure distributions are also erasure distributions with easy-to-compute Bhattacharyya parameters. It will also be apparent that this procedure yields overestimates of the true Bhattacharyya parameters. On the other hand, since there is no degradation involved after the $n$th step of this algorithm, all Bhattacharyya parameters that eventually approach zero - it follows from (3.10) below that at least a $(1-\delta)$ fraction of them will do so will be roughly $O\left(2^{-\sqrt{2^{m}}}\right)$. This follows from Theorem 2.5. By choosing $m$ sufficiently large, these can be made roughly $O\left(2^{-\sqrt{2^{m+n}}}\right)$, i.e., exponentially small in the square root of the blocklength. Therefore by varying $\delta$, the procedure above can be used to find codes with exponentially small error probabilities and rates arbitrarily close to the source entropy (respectively, channel capacity).

\subsubsection{A Special Case: Erasure Distributions}

The difficulty in computing the reliabilities of polarized bits vanishes in a special case. Consider the class of distributions with $\mathcal{Y}=\{0,1, \mathcal{E}\}$, $p(y)>0, p_{X \mid Y}(0 \mid 0)=p_{X \mid Y}(1 \mid 1)=1$, and $p_{X \mid Y}(0 \mid \mathcal{E})=p_{X \mid Y}(1 \mid \mathcal{E})=$ $1 / 2$. That is, conditioned on $Y, X$ is either constant or uniformly distributed. We will call this the class of 'erasure distributions' since it is a generalization of the case where $X$ is the uniformly distributed input to a binary erasure channel, $Y$ is the output, and $\mathcal{E}$ is the erasure symbol. The Bhattacharyya parameter and the conditional entropy for such distributions are

$$
Z(X \mid Y)=H(X \mid Y)=p(\mathcal{E}) .
$$

We will first see that polarizing $p_{X Y}$ yields distributions that are also in the erasure class. Recall that one polarization step yields the conditional distributions

$$
p\left(u_{1} \mid y_{1}^{2}\right)=\sum_{x_{1}+x_{2}=u_{1}} p\left(x_{1} \mid y_{1}\right) p\left(x_{2} \mid y_{2}\right)
$$

and

$$
p\left(u_{2} \mid y_{1}^{2} u_{1}\right)=\frac{p_{X \mid Y}\left(u_{1}+u_{2} \mid y_{1}\right) p_{X \mid Y}\left(u_{2} \mid y_{2}\right)}{p\left(u_{1} \mid y_{1}^{2}\right)} .
$$


In the first of these, notice that

$$
p\left(u_{1} \mid y_{1}^{2}\right)=\left\{\begin{array}{ll}
1 / 2, & \text { if } y_{1}=\mathcal{E} \text { or } y_{2}=\mathcal{E} \\
0 \text { or } 1, & \text { otherwise }
\end{array} .\right.
$$

That is, $p_{U_{1}\left(Y_{1}^{2}\right)}$ belongs to the erasure class, with erasure probability $2 p(\mathcal{E})-p(\mathcal{E})^{2}$. It can also be verified that

$$
p\left(u_{2} \mid y_{1}^{2} u_{1}\right)=\left\{\begin{array}{ll}
1 / 2, & \text { if } y_{1}=y_{2}=\mathcal{E} \\
0 \text { or } 1, & \text { otherwise }
\end{array},\right.
$$

which implies that $p_{U_{2}\left(Y_{1}^{2} U_{1}\right)}$ is also an erasure distribution with erasure probability $p(\mathcal{E})^{2}$. Applying the same argument to $p_{U_{1}\left(Y_{1}^{2}\right)}$ and $p_{U_{2}\left(Y_{1}^{2} U_{1}\right)}$, we see that if $p_{X Y}$ is an erasure distribution, then all of its polarized descendants are also erasure distributions. (The inverse implication is also true: if $p_{U_{1}\left(Y_{1}^{2}\right)}$ and $p_{U_{2}\left(Y_{1}^{2} U_{1}\right)}$ are erasure distributions, then so is $p_{X Y}$.) That is, unlike the general case, the effective alphabet sizes do not grow with the number of polarization levels. The Bhattacharyya parameters of the polarized distributions can then be computed by recursively applying the relations

$$
\begin{aligned}
Z\left(U_{1} \mid Y_{1}^{2}\right) & =2 Z(X \mid Y)-Z(X \mid Y)^{2}, \\
Z\left(U_{2} \mid Y_{1}^{2} U_{1}\right) & =Z(X \mid Y)^{2} .
\end{aligned}
$$

Comparing these with relations (2.12)-(2.13) for general distributions, we see that among all distributions with a given Bhattacharyya parameter, the erasure distribution has the descendants with the largest Bhattacharyya parameters. This justifies the replacement of the approximate distributions with erasure distributions in step i on page 301 .

The method above to underestimate the reliabilities can be modified to produce overestimates instead. In order to do so, one only needs to replace the degrading operation in Step 2 of the algorithm with an upgrading operation. Upgrading can be performed similarly to degrading: instead of merging symbols within each interval of the horizontal axis in Figure 3.4, one can think of each symbol in $\mathcal{Y}$ as obtained through a merging of two symbols located at the two ends of that interval, i.e., through degrading a distribution with $2|\mathcal{Y}|$ symbols, 


\section{Complexity}

all of which are located at the $2 L+1$ interval boundaries. Since two symbols that are colocated are equivalent, such a distribution can be thought of having at most $2 L+1$ symbols. Through the same arguments as above it can be shown that this operation reduces conditional entropy by at most $1 / L$, and that the total error in estimates can also be upper bounded as above. If applied recursively, this algorithm produces upgraded versions of the true distributions, and hence underestimates their Bhattacharyya parameters. A comparison of these underestimates with the overestimates produced by the degrading algorithm allows one to evaluate the rate loss incurred in quantization. See Tables 3.1 and 3.2 .

The quantization procedure we saw above is perhaps the simplest to describe and analyze, but one can think of several other methods to do quantization efficiently. One such method, given in [45], is to merge two symbols at a time until $2 L$ symbols are left. In each step, the symbols to be merged are chosen greedily so as to minimize the increase in the Bhattacharyya parameter. This variation has similar 0 figures to the algorithm we saw here - an analysis is offered in [36] — and it seems to approximate the Bhattacharyya parameters more closely. See Tables 3.1 and 3.2.

Computational complexity can be estimated separately for the two stages of the algorithm. In the first stage (Steps 0-3), each distribution $p$ created by the algorithm has at most $2 L$ symbols in $\mathcal{Y}$, and thus

Table 3.1. The highest rate $R$ for which the sum error probability of the $2^{n} R$ most reliable approximate channels (out of the $2^{n}$ ) is at most $10^{-3}$.

\begin{tabular}{lcccccc}
\hline$n$ & 5 & 8 & 11 & 14 & 17 & 20 \\
\hline Degrade & 0.1250 & 0.1836 & 0.2422 & 0.3063 & 0.3626 & 0.4051 \\
Upgrade & 0.1563 & 0.2266 & 0.3081 & 0.3730 & 0.4187 & 0.4499 \\
$n$ & 5 & 8 & 11 & 14 & 17 & 20 \\
\hline Degrade & 0.1250 & 0.2109 & 0.2969 & 0.3620 & 0.4085 & 0.4403 \\
Upgrade & 0.1250 & 0.2109 & 0.2974 & 0.3633 & 0.4102 & 0.4423 \\
\hline
\end{tabular}

Top: 'Bin and merge' algorithm discussed here. Bottom: 'Greedy mass-merging' algorithm given in [45]. Both tables are for 16 quantization levels. The underlying channel between $X$ and $Y$ is a binary symmetric channel with capacity 0.5 . 
Table 3.2. The highest rate $R$ for which the sum error probability of the $2^{n} R$ most reliable channels is at most $10^{-3}$ with $k$ quantization levels and $n=15$ recursions.

\begin{tabular}{lcccccc}
\hline$k$ & 2 & 4 & 8 & 16 & 32 & 64 \\
\hline Degrade & 0.2863 & 0.3019 & 0.3134 & 0.3264 & 0.3343 & 0.3422 \\
Upgrade & 0.4683 & 0.4221 & 0.3973 & 0.3899 & 0.3862 & 0.3838 \\
$k$ & 2 & 4 & 8 & 16 & 32 & 64 \\
\hline Degrade & 0.2895 & 0.3667 & 0.3774 & 0.3795 & 0.3799 & 0.3800 \\
Upgrade & 0.4590 & 0.3943 & 0.3836 & 0.3808 & 0.3802 & 0.3801 \\
\hline
\end{tabular}

Top: 'Bin and merge' algorithm discussed here, with $k=2 L$.

Bottom: 'Greedy mass-merging' algorithm given in [45]. The underlying channel between $X$ and $Y$ is a binary symmetric channel with capacity 0.5 .

computing $p^{-}$and $p^{+}$requires at most $O\left(L^{2}\right)$ arithmetic operations. For the degradation step, binning the $O\left(L^{2}\right)$ symbols into $2 L-1$ bins requires $O\left(L^{2}\right)$ computations. The cost of merging symbols in a bin is linear in the number of symbols, since it only involves two linear operations to compute (3.7) and (3.6). Therefore, performing Steps 1 and 2 for each distribution has time complexity $O\left(L^{2}\right)$, except for the original distribution, for which the complexity is $O\left(|\mathcal{Y}|^{2}\right)$. Since there are $2 N-1$ distributions in an $n$-level recursion tree, and since computing the Bhattacharyya parameter (or conditional entropy) for the final $N$ distributions require $O\left(L^{2}\right)$ operations, the time complexity of the first stage is $O\left(|\mathcal{Y}|^{2}\right)+O\left(N L^{2}\right)$.

The second stage (Steps i and ii) consists in recursively computing the Bhattacharyya parameters of erasure distributions using relations (3.11). These involve a constant number of arithmetic operations per polarized channel pair. Thus, with $O(N)$ initial erasure distributions, $m$ additional recursions of the second stage require $O(N M)$ operations, where $M=2^{m}$. Therefore the overall complexity is $O\left(|\mathcal{Y}|^{2}+N \log N+N M\right)$, where the blocklength is $N M$. Distributions with a continuous alphabet $\mathcal{Y}$ can be first discretized by degradation, in a similar manner as above, where binning and merging symbols is performed through integration. The issue of the computational complexity required to compute such integrals will not be discussed here.

At blocklength $M N$, if all intermediate distributions are computed in a breadth-first manner, then all intermediate distributions at level 


\section{Complexity}

$n-1$ tree need to be stored to compute the distributions at level $n$. With the additional $O(M N)$ space required to store the Bhattacharyya parameters, the space complexity of this method is $O\left(N\left(M+L^{2}\right)\right)$. Alternatively, distributions can be created in a depth-first manner. In this method, computing any polarized distribution on the tree requires storing only its ancestors. This modification reduces the space complexity to $O\left(L^{2} \log N+\log M\right)$. 


\section{Processes with Arbitrary Alphabets}

We saw in Section 2 that Arıkan's recursive method creates random variables with extremal entropies out of a binary memoryless process with moderate entropy, which allows one to construct capacityachieving channel codes as well as entropy-achieving source codes. The cause of this polarization effect is simple: if a memoryless process $\left(X_{1}, Y_{1}\right),\left(X_{2}, Y_{2}\right), \ldots$ with binary $X_{1}$ has moderate entropy $H=H\left(X_{1} \mid\right.$ $\left.Y_{1}\right) \in(\epsilon, 1-\epsilon)$, then the entropies $H^{-}=H\left(U_{1} \mid Y_{1}^{2}\right)$ and $H^{+}=H\left(U_{2} \mid\right.$ $\left.Y_{1}^{2} U_{1}\right)$ of

$$
U_{1}=X_{1}+X_{2} \quad \text { and } \quad U_{2}=X_{2}
$$

are strictly away from each other (Lemma 2.2), i.e.,

$$
H^{+}+\delta(\epsilon) \leq H \leq H^{-}-\delta(\epsilon) \text { for some } \delta(\epsilon)>0 .
$$

This is illustrated in Figure 4.1. If $H^{-}$and $H^{+}$are also moderate, applying (4.1) a second time will cause further separation in the resulting entropies. Continuing in this fashion, we see that if the 'entropy paths' we create converge at all - they indeed do - they can converge only to zero or to one, yielding polarization. It is then clear that for polarization to take place, the only requirement for a recursive transform and the underlying process is that the resulting entropies satisfy 

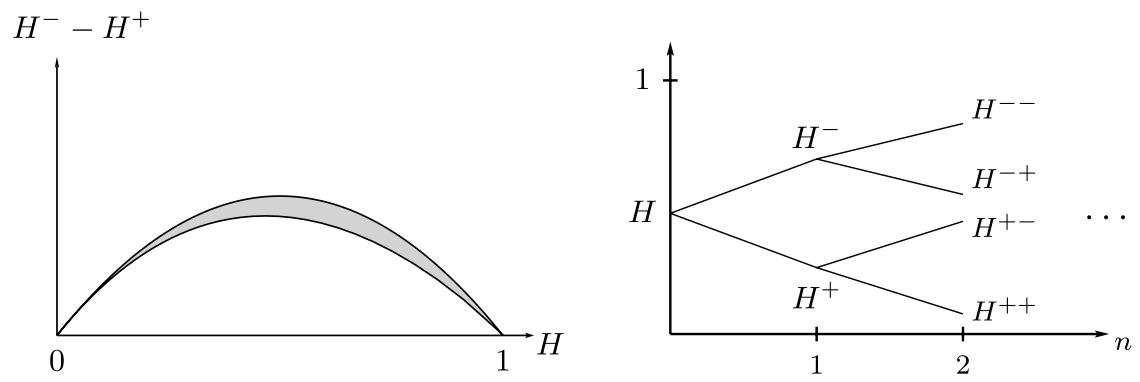

Fig. 4.1 Left: in the binary case, allowed values of the difference $H^{-}-H^{+}$versus $H$ are inside the shaded region, and are away from zero except at $H=0$ and $H=1$. Right: the entropy paths created by the recursive construction keep bifurcating until they converge to zero or one.

(4.2) at each step. This raises the following question: what classes of processes can be polarized recursively, and what types of transforms polarize these processes?

By the end of this monograph, it will become clear that polarization is a fairly general phenomenon. We will begin demonstrating this generality by showing how to polarize non-binary memoryless processes. Our motivation for this study is simple: several source and channel coding problems of practical interest are in a non-binary setting. Perhaps the most prominent example is the additive white Gaussian channel, where the coding gains achieved by using non-binary inputs can be significant.

As in the binary case, the memorylessness of the underlying processes will allow us to focus our attention on one-step transforms; once the properties of these are established, the large-blocklength behavior will readily follow. We will first discuss processes with prime alphabet sizes. As we will see, such processes can be polarized by a simple extension of Arıkan's original method. We will then establish sufficient conditions for an Arıkan-like transform to polarize processes with arbitrary alphabets, and provide an example of a transform family that satisfies these conditions for all alphabet sizes. In all cases, the speed with which polarization takes place will be as in the binary case. We will leave out the translation of these results to low-complexity polar source and channel coding schemes, as we hope that these will be evident from the exposition in Sections 2 and 3. 
Suppose $\left(X_{1}, Y_{1}\right),\left(X_{2}, Y_{2}\right), \ldots$ is an i.i.d. process, where $X_{1} \in$ $\{0, \ldots, q-1\}$, and $q$ is an arbitrary integer. As in the binary case, $Y_{1}$ takes values in a finite but arbitrary set $\mathcal{Y}$. We are interested in finding an invertible transform $G: X_{1}^{2} \rightarrow U_{1}^{2}$ for which (4.2) holds for all joint distributions on $\left(X_{1}, Y_{1}\right)$. Out of the many possibilities, perhaps the simplest guess is to use (4.1) by replacing the modulo- 2 addition with a modulo- $q$ addition. Before studying when this transform polarizes memoryless processes, it is useful to consider the following example, which shows when it does not:

Example 4.1. Let $X_{1}$ be uniformly distributed over $\mathcal{X}=\{0,1,2,3\}$ and let $Y_{1} \in\{0,1\}$ be such that $p_{Y \mid X}(0 \mid 0)=p_{Y \mid X}(0 \mid 2)=p_{Y \mid X}(1 \mid$ 1) $=p_{Y \mid X}(1 \mid 3)=1$. Then, ${ }^{1}$

$$
H\left(X_{1} \mid Y_{1}\right)=1 / 2 .
$$

Also let $U_{1}=X_{1}+X_{2}$ and $X_{2}=U_{2}$. Then, the pairs $\left(X_{1}, Y_{1}\right),\left(U_{1}, Y_{1}^{2}\right)$, and $\left(U_{2}, Y_{1}^{2} U_{1}\right)$ are identically distributed (after appropriate grouping and labelling), and therefore

$$
H\left(U_{2} \mid Y_{1}^{2} U_{1}\right)=H\left(X_{1} \mid Y_{1}\right)=H\left(U_{1} \mid Y_{1}^{2}\right)
$$

That is, the transformation has no effect on the resulting distributions. Clearly, this also implies that applying the same transform a second time (and further) will have no effect on the distributions or on the entropies.

At a first look, the anomaly in the above example may seem artificial: it is indeed easy to see that if we relabel the alphabet $\mathcal{X}$ by swapping 0 and 1 , then the equalities in (4.3) become strict inequalities. Nevertheless, renaming the symbols alone may not be sufficient for polarization, as it may not guarantee that the resulting distributions will lead to a strict separation of entropies in the further steps of the construction.

\footnotetext{
${ }^{1}$ In this and the succeeding sections, entropies will be computed with base- $q$ logarithms, and therefore will be $[0,1]$-valued. Also, addition of $q$-ary random variables will be modulo- $q$ unless stated otherwise.
} 
The difficulty illustrated in the above example is in fact common to all alphabets $\mathcal{X}$ of composite size. It is not peculiar to the particular transform in (4.1) either: suppose that $f$ is an operation for which the pair $(\mathcal{X}, f)$ is a group, and consider the mapping $\left(X_{1}, X_{2}\right) \rightarrow\left(U_{1}, U_{2}\right)$

$$
U_{1}=f\left(X_{1}, X_{2}\right), \quad U_{2}=X_{2} .
$$

Then we have

Proposition 4.1. If $q=|\mathcal{X}|$ is composite, then there exists an $\epsilon>0$ and a distribution on $\left(X_{1}, Y_{1}\right)$ for which $H\left(X_{1}, Y_{1}\right) \in(\epsilon, 1-\epsilon)$ and

$$
H\left(U_{2} \mid Y_{1}^{2} U_{1}\right)=H\left(X_{1} \mid Y_{1}\right)=H\left(U_{1} \mid Y_{1}^{2}\right) .
$$

Proof. It is known [11, p. 28] that if $q$ is composite, then the group $(\mathcal{X}, f)$ has a proper nontrivial subgroup. That is, there exists a set $S \subsetneq \mathcal{X}$ with $|S|>1$ such that $(S, f)$ is a group. Now let $Y_{1}$ be a constant random variable and $X_{1}$ be uniformly distributed over $S$. It is easy to verify that this choice of $\left(X_{1}, Y_{1}\right)$ satisfies the claim.

While the relations in (4.1) (and more generally (4.4)) fail to describe all one-to-one mappings on $\mathcal{X}^{2}$, we will focus our attention to transforms of this form. In view of Proposition 4.1, we will first restrict our attention to processes with prime $q=|\mathcal{X}|$. The reason for us to discuss the prime- $q$ case before considering arbitrary alphabet sizes is twofold: First, we will see that proving polarization is relatively simple when the construction is based on (4.1). The observations we will make to this end will also be helpful in identifying the necessary properties of a transform to polarize processes over arbitrary alphabets. Second, constructions based on (4.1) are linear. As we will see in Section 5, generalizations of linear constructions are easy to analyze, and they can lead to higher rates of polarization.

\subsection{Alphabets of Prime Size}

Let $\left(X_{1}, Y_{1}\right),\left(X_{2}, Y_{2}\right), \ldots$ be an i.i.d. process with prime $q=|\mathcal{X}|$. Define

$$
U_{1}=X_{1}+X_{2} \text { and } U_{2}=X_{2},
$$


where the addition is modulo- $q$. The next result states that the anomaly described in Example 4.1 and Proposition 4.1 vanish when $q$ is prime.

Lemma 4.2. For all $\delta>0$, there exists $\epsilon(\delta)>0$ such that if $\left(X_{1}, Y_{1}\right)$ and $\left(X_{2}, Y_{2}\right)$ are independent (but not necessarily identically distributed) pairs of random variables, then

$$
H\left(X_{1} \mid Y_{1}\right), H\left(X_{2} \mid Y_{2}\right) \in(\delta, 1-\delta),
$$

implies

$$
H\left(X_{1}+X_{2} \mid Y_{1}^{2}\right) \geq \max \left\{H\left(X_{1} \mid Y_{1}\right), H\left(X_{2} \mid Y_{2}\right)\right\}+\epsilon(\delta),
$$

provided that $q=|\mathcal{X}|$ is prime.

Before proving Lemma 4.2, let us describe the recursive construction and show that Lemma 4.2 implies polarization. These will be exactly as in the binary case: For $n=0,1, \ldots$, let $N=2^{n}$ and define a sequence of transforms $G_{n}: \mathcal{X}^{N} \rightarrow \mathcal{X}^{N}$ recursively through

$$
\begin{aligned}
& G_{0}(u)=u, \\
& G_{n}(u)=\pi_{n}\left(G_{n-1}\left(u_{1}\right)+G_{n-1}\left(u_{2}\right), G_{n-1}\left(u_{2}\right)\right), \quad n=1,2, \ldots,
\end{aligned}
$$

where $u_{1}, u_{2} \in \mathcal{X}^{N / 2}$ and $\pi_{n}: \mathcal{X}^{N} \rightarrow \mathcal{X}^{N}$ permutes the components of its argument vector through

$$
\begin{gathered}
\pi_{n}(v)_{2 i-1}=v_{i} \\
\pi_{n}(v)_{2 i}=v_{i+N / 2}
\end{gathered}, \quad i=1, \ldots, N / 2 .
$$

Now define

$$
U_{1}^{N}=G_{n}\left(X_{1}^{N}\right)
$$

As in the binary case, the transform $G_{n}$ polarizes the underlying process.

Theorem 4.3. For all $\epsilon>0$,

$$
\begin{aligned}
& \lim _{n \rightarrow \infty} \frac{1}{N}\left|\left\{i: H\left(U_{i} \mid Y_{1}^{N} U_{1}^{i-1}\right)>1-\epsilon\right\}\right|=H\left(X_{1} \mid Y_{1}\right), \\
& \lim _{n \rightarrow \infty} \frac{1}{N}\left|\left\{i: H\left(U_{i} \mid Y_{1}^{N} U_{1}^{i-1}\right)<\epsilon\right\}\right|=1-H\left(X_{1} \mid Y_{1}\right) .
\end{aligned}
$$


For the proof of the above theorem, we set the notation

$$
H\left(X_{1} \mid Y_{1}\right)^{-}:=H\left(U_{1} \mid Y_{1}^{2}\right), \quad H\left(X_{1} \mid Y_{1}\right)^{+}:=H\left(U_{2} \mid Y_{1}^{2} U_{1}\right),
$$

similarly to the binary case. We also define a $\{-,+\}$-valued i.i.d. process $B_{1}, B_{2}, \ldots$ with $\operatorname{Pr}\left[B_{1}=-\right]=1 / 2$, and a $[0,1]$-valued process $H_{0}, H_{1}, \ldots$ through

$$
\begin{aligned}
& H_{0}=H\left(X_{1} \mid Y_{1}\right) \\
& H_{n}=H_{n-1}^{B_{n}}, \quad n=1,2, \ldots
\end{aligned}
$$

Proof. It follows from the equivalences in (2.10) that

$$
\operatorname{Pr}\left[H_{n} \in \mathcal{I}\right]=\frac{1}{N}\left|\left\{i: H\left(U_{i} \mid Y_{1}^{N} U_{1}^{i-1}\right) \in \mathcal{I}\right\}\right|
$$

for all $\mathcal{I} \subseteq[0,1]$. It therefore suffices to show that for all $\epsilon>0$

$$
\begin{gathered}
\lim _{n \rightarrow \infty} \operatorname{Pr}\left[H_{n}>1-\epsilon\right]=H\left(X_{1} \mid Y_{1}\right), \\
\lim _{n \rightarrow \infty} \operatorname{Pr}\left[H_{n}<\epsilon\right]=1-H\left(X_{1} \mid Y_{1}\right) .
\end{gathered}
$$

We will show the stronger result that $H_{n}$ converges almost surely (i.e., not only in probability) to a random variable $H_{\infty}$ with $\operatorname{Pr}\left[H_{\infty}=1\right]=1-\operatorname{Pr}\left[H_{\infty}=0\right]=H\left(X_{1} \mid Y_{1}\right)$. To that end, observe that $H_{n}^{-}+H_{n}^{+}=2 H_{n}$, from which it follows that the process $H_{0}, H_{1}, \ldots$ is a bounded martingale and therefore converges almost surely to a random variable $H_{\infty}$. As almost sure convergence implies convergence in $\mathcal{L}^{1}$, we have $E\left[\left|H_{n+1}-H_{n}\right|\right]=\frac{1}{2} E\left[H_{n}^{-}-H_{n}\right]+\frac{1}{2} E\left[H_{n}-H_{n}^{+}\right]=$ $E\left[H_{n}^{-}-H_{n}\right] \rightarrow 0$. On the other hand, Lemma 4.2 implies that $H_{n}^{-}-$ $H_{n}>\delta(\epsilon)$ if $H_{n} \in(\epsilon, 1-\epsilon)$, from which it follows that $H_{n} \rightarrow\{0,1\}$ with probability 1, i.e., that $H_{\infty}$ is $\{0,1\}$-valued. The claim on the distribution of $H_{\infty}$ follows from the relation $E\left[H_{\infty}\right]=E\left[H_{0}\right]=H\left(X_{1} \mid Y_{1}\right)$.

The first proof of polarization for the non-binary case, given in [40], consisted in showing that the source Bhattacharyya parameters (defined in the next section) polarize, and that this convergence implies the convergence of the entropies. This somewhat convoluted proof is included in Appendix 4.C for the interested reader. The proof above is direct and simple once Lemma 4.2 is obtained, as it is clearly 
a verbatim reproduction of the one given in Section 2. Note, however, that Lemma 4.2 is weaker than Lemma 2.2, which identifies the distributions that are extremal in terms of how much they are polarized. Our preliminary studies suggest that such simple characterizations may not be possible in full generality in the $q$-ary case.

\subsubsection{Proof of Lemma 4.2}

We will first prove the unconditional version of Lemma 4.2, the proof for the conditional case will then follow easily. In particular, we will first show that if $X_{1}$ and $X_{2}$ are independent random variables with moderate entropies, then the entropy of $X_{1}+X_{2}$ is strictly larger than the entropy of either random variable (Lemma 4.6). To see why $q$ has to be prime for this to hold, note that $p_{X_{1}+X_{2}}$ is obtained through a cyclic convolution, i.e., by taking a weighted sum of the cyclic shifts of $p_{X_{1}}$, where the weights are given by the coefficients of $p_{X_{2}}$ (or vice versa, see Figure 4.2). These cyclic shifts are guaranteed to be away from each other only if $q$ is prime and $H\left(X_{1}\right)$ is not too large, which in turn implies that $H\left(X_{1}+X_{2}\right)$ is strictly larger than $H\left(X_{1}\right)$.

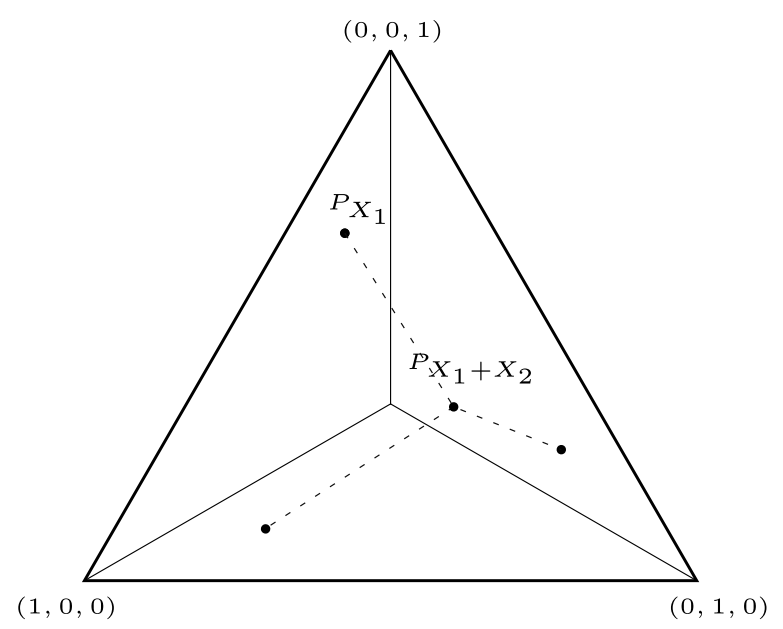

Fig. 4.2 Cyclic convolution of two probability distributions over a ternary alphabet. The corners of the triangle represent the unit mass distributions and the center represents the uniform distribution. 
We now obtain a few simple lemmas in order to formalize these arguments. Some notation first: we let both $H(p)$ and $H(X)$ denote the entropy of a random variable $X \in \mathcal{X}$ with probability distribution $p$. We let $p_{i}, i \in \mathcal{X}$ denote the cyclic shifts of $p$, i.e.,

$$
p_{i}(m)=p(m-i) .
$$

The cyclic convolution of probability distributions $p$ and $r$ will be denoted by $p * r$. That is,

$$
p * r=\sum_{i \in \mathcal{X}} p(i) r_{i}=\sum_{i \in \mathcal{X}} r(i) p_{i} .
$$

We also let $\operatorname{uni}(\mathcal{X})$ denote the uniform distribution over $\mathcal{X}$.

We first show that the $\mathcal{L}_{1}$ distance of a distribution from the uniform one is lower bounded by the corresponding Kullback-Leibler divergence. This result partially complements Pinsker's inequality.

Lemma 4.4. Let $p$ be a distribution over $\mathcal{X}$. Then,

$$
\|p-\operatorname{uni}(\mathcal{X})\|_{1} \geq \frac{1}{q \log e}[1-H(p)]
$$

Proof.

$$
\begin{aligned}
1-H(p) & =\sum_{i \in \mathcal{X}} p(i) \log \frac{p(i)}{1 / q} \\
& \leq \log e \sum_{i} p(i)\left[\frac{p(i)-1 / q}{1 / q}\right] \\
& \leq q \log e \sum_{i} p(i)|p(i)-1 / q| \\
& \leq q \log e\|p-\operatorname{uni}(\mathcal{X})\|_{1},
\end{aligned}
$$

where we used the relation $\ln t \leq t-1$ in the first inequality.

Note that Lemma 4.4 holds for distributions over arbitrary finite sets. That $|\mathcal{X}|$ is a prime number has no bearing upon the above proof. 
We next show that for prime $q$, if a distribution does not have too high an entropy, then its cyclic shifts will be away from each other:

Lemma 4.5. Let $p$ be a distribution over $\mathcal{X}$. Then,

$$
\left\|p_{i}-p_{j}\right\|_{1} \geq \frac{1-H(p)}{2 q^{2}(q-1) \log e} .
$$

for all $i, j \in \mathcal{X}, i \neq j$.

Proof. Given $i \neq j$, let $m=j-i$. We will show that there exists a $k \in \mathcal{X}$ satisfying

$$
|p(k)-p(k+m)| \geq \frac{1-H(p)}{2 q^{2}(q-1) \log e},
$$

which will yield the claim since $\left\|p_{i}-p_{j}\right\|_{1}=\sum_{k \in \mathcal{X}}|p(k)-p(k+m)|$.

Suppose that $H(p)<1$, as the claim is trivial otherwise. Let $p^{(\ell)}$ denote the $\ell$ th largest element of $p$, and let $S=\left\{\ell: p^{(\ell)} \geq \frac{1}{q}\right\}$. Note that $S$ is a proper subset of $\mathcal{X}$. We have

$$
\begin{aligned}
\sum_{\ell=1}^{|S|}\left[p^{(\ell)}-p^{(\ell+1)}\right] & =p^{(1)}-p^{(|S|+1)} \\
& \geq p^{(1)}-1 / q \\
& \geq \frac{1}{2(q-1)}\|p-\operatorname{uni}(\mathcal{X})\|_{1} \\
& \geq \frac{1-H(p)}{2 q(q-1) \log e} .
\end{aligned}
$$

In the above, the second inequality is obtained by observing that $p^{(1)}-1 / q$ is minimized when $p^{(1)}=\cdots=p^{(q-1)}$, and the third inequality follows from Lemma 4.4. Therefore, there exists at least one $\ell \in S$ such that

$$
p^{(\ell)}-p^{(\ell+1)} \geq \frac{1-H(p)}{2 q^{2}(q-1) \log e} .
$$

Given such an $\ell$, let $A=\{1, \ldots, \ell\}$. Since $q$ is prime, $\mathcal{X}$ can be written as

$$
\mathcal{X}=\{k, k+m, k+m+m, \ldots, k \underbrace{+m+\cdots+m}_{q-1 \text { times }}\}
$$


for any $k \in \mathcal{X}$ and $m \in \mathcal{X} \backslash\{0\}$. Therefore, since $A$ is a proper subset of $\mathcal{X}$, there exists a $k \in A$ such that $k+m \in A^{c}$, implying

$$
p(k)-p(k+m) \geq \frac{1-H(p)}{2 q^{2}(q-1) \log e},
$$

which yields the claim.

We can now show that unless two independent random variables are both uniformly distributed or are both constants, their modulo- $q$ addition strictly increases entropy:

Lemma 4.6. Let $A, B \in \mathcal{X}$ be two independent random variables. For all $\delta>0$, there exists $\epsilon_{1}(\delta)>0$ such that

$$
\min \{H(A), 1-H(B)\} \geq \delta
$$

implies

$$
H(A+B) \geq H(B)+\epsilon_{1}(\delta)
$$

Proof. Let $p$ and $r$ denote the probability distributions of $A$ and $B$, respectively, and let $e_{i}$ denote the distribution with a unit mass on $i \in \mathcal{X}$. Since $H(p) \geq \delta>H\left(e_{i}\right)=0$, it follows from the continuity of entropy that

$$
\min _{i}\left\|p-e_{i}\right\|_{1} \geq \mu(\delta)
$$

for some $\mu(\delta)>0$. On the other hand, since $H(r) \leq 1-\delta$, we have by Lemma 4.5 that

$$
\left\|r_{i}-r_{j}\right\|_{1} \geq \frac{\delta}{2 q^{2}(q-1) \log e}>0
$$

for all pairs $i \neq j$. Relations (4.7), (4.8), and the strict concavity of entropy implies the existence of $\epsilon_{1}(\delta)>0$ such that

$$
\begin{aligned}
H(p * r) & =H\left(\sum_{i} p(i) r_{i}\right) \\
& \geq \sum_{i} p(i) H\left(r_{i}\right)+\epsilon_{1}(\delta) \\
& =H(r)+\epsilon_{1}(\delta) .
\end{aligned}
$$


Proof of Lemma 4.2. Let $P_{1}$ and $P_{2}$ be two random probability distributions on $\mathcal{X}$, with

$$
\begin{aligned}
& P_{1}=P_{X_{1} \mid Y_{1}}\left(\cdot \mid y_{1}\right) \text { whenever } Y_{1}=y_{1}, \\
& P_{2}=P_{X_{2} \mid Y_{2}}\left(\cdot \mid y_{2}\right) \text { whenever } Y_{2}=y_{2} .
\end{aligned}
$$

It is then easy to see that

$$
\begin{aligned}
H\left(X_{1} \mid Y_{1}\right) & =\mathbb{E}\left[H\left(P_{1}\right)\right], \\
H\left(X_{2} \mid Y_{2}\right) & =\mathbb{E}\left[H\left(P_{2}\right)\right], \\
H\left(X_{1}+X_{2} \mid Y_{1}^{2}\right) & =\mathbb{E}\left[H\left(P_{1} * P_{2}\right)\right] .
\end{aligned}
$$

Suppose, without loss of generality, that $H\left(X_{1} \mid Y_{1}\right) \leq H\left(X_{2} \mid Y_{2}\right)$. We need to show that if $\mathbb{E}\left[H\left(P_{1}\right)\right], \mathbb{E}\left[H\left(P_{2}\right)\right] \in(\delta, 1-\delta)$ for some $\delta>0$, then there exists an $\epsilon(\delta)>0$ such that $\mathbb{E}\left[H\left(P_{1} * P_{2}\right)\right] \geq \mathbb{E}\left[H\left(P_{2}\right)\right]+$ $\epsilon(\delta)$. To that end, define the event

$$
C=\left\{H\left(P_{1}\right)>\delta / 2, H\left(P_{2}\right)<1-\delta / 2\right\} .
$$

Observe that

$$
\begin{aligned}
\delta & <\mathbb{E}\left[H\left(P_{1}\right)\right] \\
& \leq\left(1-\operatorname{Pr}\left[H\left(P_{1}\right)>\delta / 2\right]\right) \cdot \delta / 2+\operatorname{Pr}\left[H\left(P_{1}\right)>\delta / 2\right],
\end{aligned}
$$

implying $\operatorname{Pr}\left[H\left(P_{1}\right)>\delta / 2\right]>\frac{\delta}{2-\delta}$. It similarly follows that $\operatorname{Pr}\left[H\left(P_{2}\right)<\right.$ $1-\delta / 2]>\frac{\delta}{2-\delta}$. Note further that since $Y_{1}$ and $Y_{2}$ are independent, so are $H\left(P_{1}\right)$ and $H\left(P_{2}\right)$. Thus, the event $C$ has probability at least $\frac{\delta^{2}}{(2-\delta)^{2}}=: \epsilon_{2}(\delta)$. On the other hand, Lemma 4.6 implies that conditioned on $C$ we have

$$
H\left(P_{1} * P_{2}\right) \geq H\left(P_{2}\right)+\epsilon_{1}(\delta / 2)
$$

for some $\epsilon_{1}(\delta / 2)>0$. Thus,

$$
\begin{aligned}
\mathbb{E}\left[H\left(P_{1} * P_{2}\right)\right]= & \operatorname{Pr}[C] \cdot \mathbb{E}\left[H\left(P_{1} * P_{2}\right) \mid C\right]+\operatorname{Pr}\left[C^{c}\right] \cdot \mathbb{E}\left[H\left(P_{1} * P_{2}\right) \mid C^{c}\right] \\
\geq & \operatorname{Pr}[C] \cdot \mathbb{E}\left[H\left(P_{2}\right)+\epsilon_{1}(\delta / 2) \mid C\right] \\
& +\operatorname{Pr}\left[C^{c}\right] \cdot \mathbb{E}\left[H\left(P_{2}\right) \mid C^{c}\right] \\
\geq & \mathbb{E}\left[H\left(P_{2}\right)\right]+\epsilon_{1}(\delta / 2) \epsilon_{2}(\delta),
\end{aligned}
$$




\section{Processes with Arbitrary Alphabets}

where in the first inequality we used (4.9) and the relation $H(p * r) \geq$ $H(p)$. Setting $\epsilon(\delta):=\epsilon_{1}(\delta / 2) \epsilon_{2}(\delta)$ yields the result.

\subsubsection{Rate of Polarization}

We have seen that a similar construction to Arıkan's polarizes $q$-ary memoryless processes for prime $q$. We will now show that polarization takes place sufficiently fast - in fact as fast as in the binary case - so that source and channel codes based on such constructions have small error probability. We will do so following the approach in the binary case. For this purpose, we first need to define a reliability parameter, analogously to the Bhattacharyya parameter defined in Section 2, whose behavior through the polarization process is easy to track. For the $q$-ary case, a convenient choice turns out to be

$$
Z(X \mid Y):=\frac{1}{q-1} \sum_{\substack{x, x^{\prime} \in \mathcal{X} \\ x \neq x^{\prime}}} \sum_{y} \sqrt{p_{X Y}(x, y) p_{X Y}\left(x^{\prime}, y\right)}
$$

It is easy to see that this parameter takes values in $[0,1]$. As a measure of reliability, it is natural to expect that $Z(X \mid Y)$ upper bound the average error probability of the optimal decoder, and that

$$
\begin{aligned}
& Z(X \mid Y) \approx 1 \text { if and only if } H(X \mid Y) \approx 1, \\
& Z(X \mid Y) \approx 0 \text { if and only if } H(X \mid Y) \approx 0 .
\end{aligned}
$$

The following propositions show that these requirements are indeed met:

Proposition 4.7. $P_{\mathrm{e}}(X \mid Y) \leq(q-1) Z(X \mid Y)$.

Proof. Let $P_{e, x}$ denote the error probability of the optimal decision rule conditioned on $X=x$. We have

$$
\begin{aligned}
P_{\mathrm{e}, x} & \leq \sum_{y} p(y \mid x) \mathbb{1}_{\left[\exists x^{\prime} \neq x: p_{X \mid Y}\left(x^{\prime} \mid y\right) \geq p_{X \mid Y}(x \mid y)\right]} \\
& \leq \sum_{y} p(y \mid x) \sum_{x^{\prime}: x^{\prime} \neq x} \mathbb{1}_{\left[p_{X \mid Y}\left(x^{\prime} \mid y\right) \geq p_{X \mid Y}(x \mid y)\right]}
\end{aligned}
$$




$$
\begin{aligned}
& \leq \sum_{x^{\prime}: x^{\prime} \neq x} \sum_{y} \frac{p_{X \mid Y}(x \mid y) p(y)}{p(x)} \sqrt{\frac{p_{X \mid Y}\left(x^{\prime} \mid y\right)}{p_{X \mid Y}(x \mid y)}} \\
& =\sum_{x^{\prime}: x^{\prime} \neq x} \sum_{y} \frac{1}{p(x)} \sqrt{p_{X Y}\left(x^{\prime}, y\right) p_{X Y}(x, y)} .
\end{aligned}
$$

Averaging the above relation over $x$ yields the claim.

Proposition 4.8.

$$
\begin{aligned}
Z(X \mid Y)^{2} & \leq H(X \mid Y) \\
H(X \mid Y) & \leq \log (1+(q-1) Z(X \mid Y)) .
\end{aligned}
$$

Proof. See Appendix 4.A.

Since the polarization construction is recursive as in the binary case, the limiting behavior of the $Z$ parameters along the polarization process is determined by their one-step behavior. In particular, the following bounds will suffice to conclude that polarization takes place fast:

Lemma 4.9. Let $f: \mathcal{X}^{2} \rightarrow \mathcal{X}$ be such that both functions $f\left(x_{1}, \cdot\right)$ : $\mathcal{X} \rightarrow \mathcal{X}$ and $f\left(\cdot, x_{2}\right): \mathcal{X} \rightarrow \mathcal{X}$ are invertible for all $x_{1}$ and $x_{2}$, respectively. Defining $V_{1}:=f\left(X_{1}, X_{2}\right)$ and $V_{2}:=X_{2}$ we have

$$
\begin{aligned}
Z\left(V_{1} \mid Y_{1}^{2}\right) & \leq\left(q^{2}-q+1\right) Z\left(X_{1} \mid Y_{1}\right) \\
Z\left(V_{2} \mid Y_{1}^{2} V_{1}\right) & \leq(q-1) Z\left(X_{1} \mid Y_{1}\right)^{2} .
\end{aligned}
$$

Clearly, bounds that are relevant to the present case are obtained by taking $f$ to be the modulo- $q$ addition. The reason for us to state these bounds in a slightly more general setting will be evident when we consider polarization for arbitrary alphabet sizes in the next section. 
Proof. The assumptions on the function $f$ imply that there exist $q$ permutations $\pi_{i}: \mathcal{X} \rightarrow \mathcal{X}, i=0, \ldots, q-1$ with

$$
\pi_{i}(x) \neq \pi_{j}(x) \text { for all } i \neq j, x \in \mathcal{X}
$$

such that $\pi_{i}(j)=f(j, i)$. We therefore have

$$
p\left(v_{1}, v_{2}, y_{1}, y_{2}\right)=p_{X Y}\left(\pi_{v_{2}}^{-1}\left(v_{1}\right), y_{1}\right) p_{X Y}\left(v_{2}, y_{2}\right) .
$$

To obtain the first claim, we write

$$
\begin{aligned}
Z\left(V_{1} \mid Y_{1}^{2}\right)= & \frac{1}{q-1} \sum_{\substack{v_{1}, v_{1}^{\prime}: \\
v_{1} \neq v_{1}^{\prime}}} \sum_{y_{1}^{2}}\left[p\left(v_{1}, y_{1}, y_{2}\right) p\left(v_{1}^{\prime}, y_{1}, y_{2}\right)\right]^{1 / 2} \\
= & \frac{1}{q-1} \sum_{\substack{v_{1}, v_{1}^{\prime}: \\
v_{1} \neq v_{1}^{\prime}}} \sum_{1}^{2}\left[\sum_{v_{2}} p\left(v_{1}, v_{2}, y_{1}, y_{2}\right) \sum_{v_{2}^{\prime}} p\left(v_{1}^{\prime}, v_{2}^{\prime}, y_{1}, y_{2}\right)\right]^{1 / 2} \\
\leq & \frac{1}{q-1} \sum_{v_{1}, v_{1}^{\prime}:} \sum_{v_{1} \neq v_{1}^{\prime}} \sum_{v_{2}, v_{2}^{\prime}}\left[p\left(v_{1}, v_{2}, y_{1}, y_{2}\right) p\left(v_{1}^{\prime}, v_{2}^{\prime}, y_{1}, y_{2}\right)\right]^{1 / 2} \\
= & \left.\frac{1}{q-1} \sum_{\substack{v_{2}, v_{2}^{\prime} \\
y_{2}}} \sum_{y_{X Y}}\left[p_{2}, y_{2}\right) p_{X Y}\left(v_{2}^{\prime}, y_{2}\right)\right]^{1 / 2} \\
& \cdot \sum_{\substack{v_{1}, v_{1}^{\prime}: \\
v_{1} \neq v_{1}^{\prime}}} \sum_{y_{1}}\left[p_{X Y}\left(\pi_{v_{2}}^{-1}\left(v_{1}\right), y_{1}\right) p_{X Y}\left(\pi_{v_{2}^{\prime}}^{-1}\left(v_{1}^{\prime}\right), y_{1}\right)\right]^{1 / 2} .
\end{aligned}
$$

Splitting the summation over $\left(v_{2}, v_{2}^{\prime}\right)$ into two parts $v_{2}=v_{2}^{\prime}$ and $v_{2} \neq v_{2}^{\prime}$, and considering the first part we have

$$
\begin{aligned}
& \sum_{v_{2}=v_{2}^{\prime}} \sum_{y_{2}}\left[p_{X Y}\left(v_{2}, y_{2}\right) p_{X Y}\left(v_{2}^{\prime}, y_{2}\right)\right]^{1 / 2} \\
& \cdot \frac{1}{q-1} \sum_{\substack{v_{1}, v_{1}^{\prime}: \\
v_{1} \neq v_{1}^{\prime}}} \sum_{y_{1}}\left[p_{X Y}\left(\pi_{v_{2}}^{-1}\left(v_{1}\right), y_{1}\right) p_{X Y}\left(\pi_{v_{2}^{\prime}}^{-1}\left(v_{1}^{\prime}\right), y_{1}\right)\right]^{1 / 2} .
\end{aligned}
$$

The sums on the second line above are equivalent to $Z\left(X_{1} \mid Y_{1}\right)$ for all $v_{2}$ and $y_{2}$, and those on the first line add to 1 . Therefore the above 
term is equal to $Z\left(X_{1} \mid Y_{1}\right)$. On the other hand, when $v_{2} \neq v_{2}^{\prime}$ we have

$$
\begin{aligned}
& \frac{1}{q-1} \sum_{\begin{array}{c}
v_{2}, v_{2}^{\prime}: \\
v_{2} \neq v_{2}^{\prime}
\end{array}} \sum_{y_{2}}\left[p_{X Y}\left(v_{2}, y_{2}\right) p_{X Y}\left(v_{2}^{\prime}, y_{2}\right)\right]^{1 / 2} \\
& \quad \cdot \sum_{\begin{array}{c}
v_{1}, v_{1}^{\prime}: \\
v_{1} \neq v_{1}^{\prime}
\end{array}} \sum_{y_{1}}\left[p_{X Y}\left(\pi_{v_{2}}^{-1}\left(v_{1}\right), y_{1}\right) p_{X Y}\left(\pi_{v_{2}^{\prime}}^{-1}\left(v_{1}^{\prime}\right), y_{1}\right)\right]^{1 / 2} .
\end{aligned}
$$

Here, the summation over $y_{1}$ is upper bounded by 1 , and the upper sums are equal to $Z\left(X_{1} \mid Y_{1}\right)$. Therefore the above term is upper bounded by $q(q-1) Z\left(X_{1} \mid Y_{1}\right)$. Combining this with the first part yields (4.12). To obtain (4.13), we write

$$
\begin{aligned}
Z\left(V_{2} \mid Y_{1}^{2} V_{1}\right)= & \frac{1}{q-1} \sum_{\substack{v_{2}, v_{2}^{\prime} \\
v_{2} \neq v_{2}^{\prime}}} \sum_{1}^{2}, v_{1} \\
& {\left[p_{X Y}\left(\pi_{v_{2}}^{-1}\left(v_{1}\right), y_{1}\right) p_{X Y}\left(v_{2}, y_{2}\right)\right.} \\
= & \left.\left.\frac{1}{q-1} \sum_{\substack{v_{2}, v_{2}^{\prime} \\
v_{2} \neq v_{2}^{\prime}}} \sum_{y_{2}}\left[p_{X Y}\left(v_{1}\right), y_{1}\right) p_{X Y}\left(v_{2}^{\prime}, y_{2}\right)\right]^{1 / 2} p_{X Y}\left(v_{2}^{\prime}, y_{2}\right)\right]^{1 / 2} \\
& \cdot \sum_{v_{1}} \sum_{y_{1}}\left[p_{X Y}\left(\pi_{v_{2}}^{-1}\left(v_{1}\right), y_{1}\right) p_{X Y}\left(\pi_{v_{2}^{\prime}}^{-1}\left(v_{1}\right), y_{1}\right)\right]^{1 / 2} .
\end{aligned}
$$

For all $v_{2} \neq v_{2}^{\prime}$ and $y_{2}$, the lower sums on the second line are upper bounded by $(q-1) Z\left(X_{1} \mid Y_{1}\right)$, and those on the first are equivalent to $Z\left(X_{1} \mid Y_{1}\right)$. This yields the second claim.

We are now ready to state and prove the main result on the rate of polarization:

Theorem 4.10. For all $0<\beta<1 / 2$,

$$
\lim _{n \rightarrow \infty} \frac{1}{N}\left|\left\{i: Z\left(U_{i} \mid Y_{1}^{N} U_{1}^{i-1}\right) \leq 2^{-N^{\beta}}\right\}\right|=1-H\left(X_{1} \mid Y_{1}\right)
$$


Proof. The proof is identical to that of Theorem 2.5: set the shorthand notation

$$
Z\left(X_{1} \mid Y_{1}\right)^{-}:=Z\left(U_{1} \mid Y_{1}^{2}\right), \quad Z\left(X_{1} \mid Y_{1}\right)^{+}:=Z\left(U_{2} \mid Y_{1}^{2} U_{1}\right) .
$$

Define a $\{-,+\}$-valued i.i.d. process $B_{1}, B_{2}, \ldots$ with $\operatorname{Pr}\left[B_{1}=-\right]=1 / 2$ and a $[0,1]$-valued process $Z_{0}, Z_{1}, \ldots$ with

$$
\begin{aligned}
& Z_{0}=Z\left(X_{1} \mid Y_{1}\right) \\
& Z_{n}=Z_{n-1}^{B_{n}}, \quad n=1,2, \ldots .
\end{aligned}
$$

Then, the equivalences in (2.14) imply that

$$
\operatorname{Pr}\left[Z_{n} \in \mathcal{I}\right]=\frac{1}{N}\left|\left\{i: Z\left(U_{i} \mid Y_{1}^{N} U_{1}^{i-1}\right) \in \mathcal{I}\right\}\right|
$$

for all $\mathcal{I} \subseteq[0,1]$. Further, recall that the process $H_{0}, H_{1}, \ldots$ defined in (4.6) converges almost surely to the set $\{0,1\}$ (see proof of Theorem 4.3). It then follows from Proposition 4.8 that the process $Z_{0}, Z_{1}, \ldots$ also converges almost surely to the set $\{0,1\}$ with $\operatorname{Pr}\left[\lim _{n \rightarrow \infty} Z_{n}=0\right]=$ $1-H\left(X_{1} \mid Y_{1}\right)$. The claim then follows from Lemma 2.10 by taking $\mathcal{I}=\left[0,2^{-N^{\beta}}\right]$.

\subsection{Arbitrary Finite Alphabets}

We saw in the previous section that the mapping $\left(X_{1}, X_{2}\right) \rightarrow\left(X_{1}+\right.$ $\left.X_{2}, X_{2}\right)$ fails to polarize certain processes whenever $q=|\mathcal{X}|$ is a composite number (Example 4.1). We also saw that the difficulty with such alphabets persists so long as ' + ' is replaced by any group operation over $\mathcal{X}$ (Proposition 4.1). We are now interested in finding transforms $\left(X_{1}, X_{2}\right) \rightarrow\left(U_{1}, U_{2}\right)$ that will polarize all i.i.d. processes over all finite alphabets. We will in particular study mappings of the form

$$
\begin{aligned}
& U_{1}=f\left(X_{1}, X_{2}\right) \\
& U_{2}=X_{2},
\end{aligned}
$$

for some $f: \mathcal{X}^{2} \rightarrow \mathcal{X}$. While not all one-to-one mappings $\left(X_{1}, X_{2}\right) \rightarrow$ $\left(U_{1}, U_{2}\right)$ can be reduced to this form, we restrict our attention to these due to their relative simplicity. 
Once we find an appropriate transform $f$, we will use it recursively as in the binary case. That is, we will define for all $n=0,1, \ldots$ and $N=2^{n}$ a sequence of transforms $G_{n}: \mathcal{X}^{N} \rightarrow \mathcal{X}^{N}$ through

$$
\begin{aligned}
G_{0}(u) & =u \\
G_{n}\left(u_{1}, u_{2}\right) & =\pi_{n}\left(f\left(G_{n-1}\left(u_{1}\right), G_{n-1}\left(u_{2}\right)\right), G_{n-1}\left(u_{2}\right)\right), \quad n=1,2, \ldots .
\end{aligned}
$$

where $u_{1}, u_{2} \in \mathcal{X}^{N / 2}$, the action of $f$ on its arguments is componentwise as in (4.15), and the permutation $\pi_{n}$ is as in the previous sections. Let us now introduce the notion of a polarizing mapping:

Definition 4.1. We call a mapping $f: \mathcal{X}^{2} \rightarrow \mathcal{X}$ polarizing if

(p.i) for all $x_{2} \in \mathcal{X}$, the mapping $x_{1} \rightarrow f\left(x_{1}, x_{2}\right)$ is invertible,

(p.ii) for all $x_{1} \in \mathcal{X}$, the mapping $x_{2} \rightarrow f\left(x_{1}, x_{2}\right)$ is invertible, ${ }^{2}$ and

(p.iii) for all $2 \leq K \leq q-1$ and distinct $a_{0}, \ldots, a_{K-1} \in \mathcal{X}$, the matrix

$$
B_{i j}=f\left(a_{i}, a_{j}\right), \quad i, j=0, \ldots, K-1
$$

has at least $K+1$ distinct entries.

Example 4.2. Consider a matrix $F$ with $F_{i j}=f(i, j), i, j=0, \ldots q-1$. (That is, $F$ is the Cayley table of $f$.) Then it is easy to see that, of the operations corresponding to

$$
F=\left[\begin{array}{lll}
0 & 1 & 2 \\
1 & 2 & 0 \\
2 & 0 & 1
\end{array}\right], \quad G=\left[\begin{array}{llll}
0 & 1 & 2 & 3 \\
1 & 2 & 3 & 0 \\
2 & 3 & 0 & 1 \\
3 & 0 & 1 & 2
\end{array}\right],
$$

$F$ is polarizing, whereas $G$ is not, since $G_{00}=G_{22}=0$ and $G_{02}=$ $G_{20}=2$, violating (p.iii). Note that $F$ and $G$ correspond to modulo-3 and modulo-4 addition, respectively (see also Example 4.1).

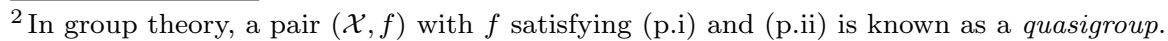


In the rest of this section, we will give meaning to Definition 4.1 by showing that the construction in (4.16) leads to polarization if $f$ is a polarizing mapping: (p.i) guarantees that the one-step transform in (4.15) is one-to-one, and (p.iii) guarantees that anomalous distributions such as the one in Example 4.1 are also polarized; it turns out that this is indeed the only type of irregularity that needs handling. Condition (p.ii) is in fact not necessary for polarization to take place, and can be relaxed. We include it Definition 4.1 only because it helps simplify the proofs. This condition is also not a very restrictive one; there are several simple families of mappings that satisfy (p.i)-(p.iii) for all alphabet sizes. We give one example here:

Example 4.3. The mapping $f\left(x_{1}, x_{2}\right)=x_{1}+\pi\left(x_{2}\right)$, where $\pi: \mathcal{X} \rightarrow \mathcal{X}$ is the permutation

$$
\pi(x)= \begin{cases}\lfloor q / 2\rfloor, & \text { if } x=0 \\ x-1, & \text { if } 1 \leq x \leq\lfloor q / 2\rfloor \\ x, & \text { otherwise }\end{cases}
$$

is polarizing for all $q=|\mathcal{X}|$. A proof of this is given in Appendix 4.B. The Cayley table of $f$ is given below for $q=6$.

$$
\left[\begin{array}{llllll}
3 & 0 & 1 & 2 & 4 & 5 \\
4 & 1 & 2 & 3 & 5 & 0 \\
5 & 2 & 3 & 4 & 0 & 1 \\
0 & 3 & 4 & 5 & 1 & 2 \\
1 & 4 & 5 & 0 & 2 & 3 \\
2 & 5 & 0 & 1 & 3 & 4
\end{array}\right]
$$

Before proceeding to the proof of polarization, let us introduce a definition in order to capture the anomaly described in Example 4.1: given a distribution $p$ over $\mathcal{X}$, let $a_{i}, i=0, \ldots, q-1$ be any labelling of the elements of $\mathcal{X}$ for which $p\left(a_{0}\right) \geq p\left(a_{1}\right) \geq \ldots \geq p\left(a_{q-1}\right)$. For all $\nu>0$, let

$$
K_{\nu}:=\min \left\{i \leq q-2: p\left(a_{i}\right)-p\left(a_{i+1}\right)>\nu\right\} \cup\{q-1\}
$$

and define

$$
M_{p, \nu}:=\left\{a_{0}, \ldots, a_{K_{\nu}}\right\}
$$


The general form of the anomaly described in Proposition 4.1 can be stated as $M_{p_{X_{1}, \nu}}=M_{p_{X_{2}}, \nu}$ for random variables $X_{1}$ and $X_{2}$. The next lemma shows that a polarizing mapping will strictly increase entropy even under such irregularities:

Lemma 4.11. For all $\epsilon, \nu>0$, there exists $\delta(\epsilon, \nu)>0$ such that if $X_{1}, X_{2} \in \mathcal{X}$ are independent random variables with $H\left(X_{1}\right), H\left(X_{2}\right) \in$ $(\epsilon, 1-\epsilon)$ and $M_{p_{X_{1}}, \nu}=M_{p_{X_{2}}, \nu}=M$ for some $M$ with $1 \leq|M| \leq q-1$, and if $f$ is a polarizing mapping, then

$$
H\left(f\left(X_{1}, X_{2}\right)\right) \geq H\left(X_{i}\right)+\delta(\epsilon, \nu), \quad i=1,2 .
$$

Proof. We will prove the claim for $i=2$, the proof for $i=1$ follows similarly by the symmetry in the assumptions. It follows from (p.ii) that there exist $q$ distinct permutations $\pi_{i}: \mathcal{X} \rightarrow \mathcal{X}, i=0, \ldots, q-1$ such that $f(j, i)=\pi_{i}(j)$. Observe also that (p.i) implies

$$
\pi_{i}(x) \neq \pi_{j}(x) \quad \text { for all } i \neq j, x \in \mathcal{X} .
$$

Defining probability distributions $r_{i}$ through $r_{i}(u)=p_{X_{2}}\left(\pi_{i}^{-1}(u)\right)$, we have

$$
p_{f\left(X_{1}, X_{2}\right)}=\sum_{i=0}^{q-1} p_{X_{1}}(i) r_{i} .
$$

It suffices to show that there exist $a, b \in \mathcal{X}$ for which

(i) $p_{X_{1}}(a), p_{X_{1}}(b) \geq \eta(\epsilon, \nu)$ for some $\eta(\epsilon, \nu)>0$, and

(ii) $\left\|r_{a}-r_{b}\right\|_{1} \geq \nu$,

since the claim will then follow immediately from (4.18), the strict concavity of entropy, and that $H\left(r_{i}\right)=H\left(X_{2}\right)$ for all $i$.

First consider the case $M=\{a\}$ for some $a \in \mathcal{X}$, and observe that $H\left(X_{1}\right)>\epsilon$ implies $p_{X_{1}}(a) \geq p_{X_{1}}(b) \geq \eta(\epsilon)$ for some $b \neq a$ and $\eta(\epsilon)>0$, satisfying (i). It also follows from $(4.17)$ that $r_{a}\left(\pi_{a}(a)\right)-r_{b}\left(\pi_{a}(a)\right)=$ $p_{X_{1}}(a)-p_{X_{1}}(c)$ for some $c \neq a$, implying (ii) since the latter difference is at least $\nu$, and therefore yielding the claim. 
Suppose now that $2 \leq|M| \leq q-1$. Define, for all $x \in \mathcal{X}$ and $T \subset \mathcal{X}$, the sets

$$
S_{x, T}=\left\{i: \pi_{x}^{-1}(i) \in T\right\},
$$

and observe that (p.iii) implies that

$$
\forall T \subset \mathcal{X}, 2 \leq|T| \leq q-1, \exists a, b \in T \quad \text { such that } S_{a, T} \neq S_{b, T} .
$$

Now let $a, b \in M$ be such that $S_{a, M} \neq S_{b, M}$. It then follows from the definition of $M$ that there exists $x \in \mathcal{X}$ for which $\left|r_{a}(x)-r_{b}(x)\right| \geq \nu$, satisfying (ii). That (i) is also satisfied can be seen by noting that $|M| \leq q-1$ and $a, b \in M$ imply $p_{X_{2}}(a), p_{X_{2}}(b) \geq \nu$. This concludes the proof.

We are now ready to prove the main result of this section, which will lead to a polarization theorem for arbitrary discrete alphabets.

Theorem 4.12. For all $\epsilon>0$, there exists $\delta(\epsilon)>0$ such that if $\left(X_{1}, Y_{1}\right),\left(X_{2}, Y_{2}\right)$ are i.i.d. random variable pairs with $H\left(X_{1} \mid Y_{1}\right) \in$ $(\epsilon, 1-\epsilon)$, and if $f: \mathcal{X}^{2} \rightarrow \mathcal{X}$ is a polarizing mapping, then

$$
H\left(f\left(X_{1}, X_{2}\right) \mid Y_{1}^{2}\right) \geq H\left(X_{1} \mid Y_{1}\right)+\delta(\epsilon) .
$$

Proof. Let $H_{1}, H_{2}$ and $H_{u}$ be [0,1]-valued random variables with

$$
\begin{aligned}
& H_{1}=H\left(X_{1} \mid Y_{1}=y_{1}\right) \\
& H_{2}=H\left(X_{2} \mid Y_{2}=y_{2}\right) \\
& H_{u}=H\left(f\left(X_{1}, X_{2}\right) \mid Y_{1}=y_{1}, Y_{2}=y_{2}\right)
\end{aligned}
$$

whenever $\left(Y_{1}, Y_{2}\right)=\left(y_{1}, y_{2}\right)$. Clearly, $H_{1}$ and $H_{2}$ are i.i.d. with

$$
E\left[H_{1}\right]=E\left[H_{2}\right]=H\left(X_{1} \mid Y_{1}\right) .
$$

Suppose first that $\operatorname{Pr}\left[H_{1} \leq \epsilon / 2\right], \operatorname{Pr}\left[H_{1} \geq 1-\epsilon / 2\right] \geq \epsilon / 2(2-\epsilon)$. Then, the event

$$
A=\left\{y_{1}, y_{2}: H_{1} \leq \epsilon / 2, H_{2} \geq 1-\epsilon / 2\right\}
$$


has probability at least $[\epsilon / 2(2-\epsilon)]^{2}$. Further, as both functions $x_{1} \rightarrow$ $f\left(x_{1}, x_{2}\right)$ and $x_{2} \rightarrow f\left(x_{1}, x_{2}\right)$ are invertible for all $x_{2}$ and $x_{1}$ respectively, we have $H_{u} \geq H_{1}, H_{2}$ for all $\left(Y_{1}, Y_{2}\right)=\left(y_{1}, y_{2}\right)$. Thus,

$$
\begin{aligned}
H\left(f\left(X_{1}, X_{2}\right) \mid Y_{1} Y_{2}\right) & =E\left[H_{u}\right] \\
& =\operatorname{Pr}[A] \cdot E\left[H_{u} \mid A\right]+\operatorname{Pr}\left[A^{c}\right] \cdot E\left[H_{u} \mid A^{c}\right] \\
& \geq \operatorname{Pr}[A] \cdot E\left[H_{2} \mid A\right]+\operatorname{Pr}\left[A^{c}\right] \cdot E\left[H_{1} \mid A^{c}\right] \\
& \geq \operatorname{Pr}[A] \cdot E\left[H_{1}+1-\epsilon \mid A\right]+\operatorname{Pr}\left[A^{c}\right] \cdot E\left[H_{1} \mid A^{c}\right] \\
& \geq E\left[H_{1}\right]+\left[\frac{\epsilon}{2(2-\epsilon)}\right]^{2}(1-\epsilon) \\
& =H\left(X_{1} \mid Y_{1}\right)+\left[\frac{\epsilon}{2(2-\epsilon)}\right]^{2}(1-\epsilon),
\end{aligned}
$$

yielding the claim.

Now suppose instead that $\operatorname{Pr}\left[H_{1} \leq \epsilon / 2\right]<\frac{\epsilon}{2(2-\epsilon)}$. Then, since

$$
\operatorname{Pr}\left[H_{1} \geq 1-\epsilon / 2\right] \leq \frac{E\left[H_{1}\right]}{1-\epsilon / 2} \leq \frac{2-2 \epsilon}{2-\epsilon},
$$

it follows that

$$
\operatorname{Pr}\left[H_{1} \in(\epsilon / 2,1-\epsilon / 2)\right] \geq \frac{\epsilon}{2(2-\epsilon)} .
$$

A similar argument shows that the above inequality also holds when $\operatorname{Pr}\left[H_{1} \geq 1-\epsilon / 2\right]<\frac{\epsilon}{2(2-\epsilon)}$. We will now show that the conditions of Lemma 4.11 hold with positive probability whenever we have (4.20). For that purpose, note that it follows from Lemma 4.4 that for all $\epsilon>0$, there exists $\nu(\epsilon)>0$ for which $H(V) \leq 1-\epsilon / 2$ implies $\left|M_{p_{V}, \nu}\right| \leq q-1$. Given such a $\nu$, let $S_{1} \subset \mathcal{X}$ and $S_{2} \subset \mathcal{X}$ be random sets with

$$
\begin{aligned}
& S_{1}=M_{p_{X_{1} \mid Y_{1}=y_{1}}, \nu}, \quad \text { whenever } Y_{1}=y_{1} \\
& S_{2}=M_{p_{X_{2} \mid Y_{2}=y_{2}}, \nu}, \quad \text { whenever } Y_{2}=y_{2} .
\end{aligned}
$$

As $S_{1}$ and $S_{2}$ are independent and identically distributed, it follows from (4.20) and the above argument that there exists $S \subset \mathcal{X}$ with $1 \leq$ $|S| \leq q-1$ such that the event

$$
B=\left\{y_{1}, y_{2}: S_{1}=S_{2}=S\right\}
$$


has probability at least $\left[\epsilon / 2^{q}(2-\epsilon)\right]^{2}$. It then follows from Lemma 4.11 that $H_{u} \geq H_{1}+\delta(\epsilon, \nu(\epsilon))$ for some $\delta(\epsilon, \nu(\epsilon))>0$ whenever $y_{1}, y_{2} \in B$. Therefore

$$
\begin{aligned}
E\left[H_{u}\right] & =\operatorname{Pr}[B] \cdot E\left[H_{u} \mid B\right]+\operatorname{Pr}\left[B^{c}\right] \cdot E\left[H_{u} \mid B^{c}\right] \\
& \geq \operatorname{Pr}[B] \cdot E\left[H_{1}+\delta(\epsilon, \nu(\epsilon)) \mid B\right]+\operatorname{Pr}\left[B^{c}\right] \cdot E\left[H_{1} \mid B^{c}\right] \\
& =E\left[H_{1}\right]+\left[\epsilon / 2^{q}(2-\epsilon)\right]^{2} \cdot \delta(\epsilon, \nu(\epsilon)),
\end{aligned}
$$

completing the proof.

We can now state the polarization theorem for arbitrary finite alphabets. Let $\left(X_{1}, Y_{1}\right),\left(X_{2}, Y_{2}\right), \ldots$ be a discrete, i.i.d. process with $|\mathcal{X}|<\infty$. Also let $f$ be a polarizing mapping, and define

$$
U_{1}^{N}=G_{n}\left(X_{1}^{N}\right)
$$

where $G_{n}$ is as in (4.16). We have

Theorem 4.13. For all $\epsilon>0$,

$$
\begin{aligned}
& \lim _{n \rightarrow \infty} \frac{1}{N}\left|\left\{i: H\left(U_{i} \mid Y_{1}^{N} U_{1}^{i-1}\right)>1-\epsilon\right\}\right|=H\left(X_{1} \mid Y_{1}\right), \\
& \lim _{n \rightarrow \infty} \frac{1}{N}\left|\left\{i: H\left(U_{i} \mid Y_{1}^{N} U_{1}^{i-1}\right)<\epsilon\right\}\right|=1-H\left(X_{1} \mid Y_{1}\right) .
\end{aligned}
$$

Proof. The proof follows from Theorem 4.12, and is identical to those of Theorems 2.3 and 4.3 .

The rate of polarization for the construction in (4.16) is also as in the binary case:

Theorem 4.14. For all $0<\beta<1 / 2$,

$$
\lim _{n \rightarrow \infty}\left|\left\{i: Z\left(U_{i} \mid Y_{1}^{N} U_{1}^{i-1}\right) \leq 2^{-N^{\beta}}\right\}\right|=1-H\left(X_{1} \mid Y_{1}\right)
$$

Proof. The proof follows from Lemma 4.9 and is identical to that of Theorem 4.10. 


\subsection{How to Achieve Capacity}

Polarization results in this section immediately yield polar source coding methods that compress any discrete memoryless source to its entropy. Recall from the discussion in Section 2.4, however, that translating polarization results to channel coding schemes becomes trivial only for uniformly distributed channel inputs. Clearly, this statement is equally valid for channels with non-binary input alphabets. Therefore one can achieve the symmetric capacity of discrete memoryless channels with the methods discussed so far, as opposed to the true capacity. In channels where the gap between these two rates is significant, one can use the following generic method, discussed in [14, p. 208], to approach the true capacity: Given a channel $W: \mathcal{X} \rightarrow \mathcal{Y}$, one can construct a new channel $W^{\prime}: \mathcal{X}^{\prime} \rightarrow \mathcal{Y}$ with $\left|\mathcal{X}^{\prime}\right| \geq|\mathcal{X}|$, where $W^{\prime}\left(y \mid x^{\prime}\right)=W\left(y \mid f\left(x^{\prime}\right)\right)$ and $f: \mathcal{X}^{\prime} \rightarrow \mathcal{X}$ is a deterministic map. Note that the mutual informations $I(X ; Y)$ and $I\left(X^{\prime} ; Y\right)$ developed across $W$ and $W^{\prime}$ respectively are identical for any distribution on input $X^{\prime}$ to $W^{\prime}$ and the induced distribution on $X$. Observe further that if $X^{\prime}$ is uniformly distributed, then one can induce, using an appropriate mapping $f$, any distribution $p_{X}$ on $X$ with $p_{X}(x)=k_{x} /\left|\mathcal{X}^{\prime}\right|$, where $k_{x}$ 's are integer-valued. Consequently, one can approach the true capacity of any discrete memoryless channel $W$ by choosing $f$ so as to approximate the capacity-achieving input distribution of this channel, and using a symmetric capacityachieving polar code for the created channel $W^{\prime}$. Using large input alphabets increases the code complexity, however, as we will see next.

\subsection{Complexity}

Non-binary codes based on the polarization transforms discussed in this section will have low-complexities like their binary counterparts. In particular, if one assumes that the computation of a one-step polarizing mapping takes one unit of time, then the time and space complexity of encoding these codes will be $O(N \log N)$ in the blocklength. Similarly, it readily follows from the results in [4] that successive cancellation decoding with such codes can be performed with $O\left(q^{2} N \log N\right)$ time and $O(q N \log N)$ space complexities. Also by a straightforward extension of 
the algorithm proposed in [45], these codes can be constructed with $O\left(q^{2} N\right)$ time and $O(q \log N)$ space complexities.

In the next section, we will continue studying the generality of polarization. In particular, we will show that memoryless processes can be polarized by generalizations of Arıkan's construction. We will see that for non-binary processes, such generalizations can produce substantial gains in error probability without too much added complexity.

\section{A Proof of Proposition 4.8}

Proof of (4.10). The proof of this inequality was given in [5] for the binary case; the proof of the $q$-ary version is identical. We nevertheless include it here for completeness.

The Rényi entropy of order $\alpha$ of a random variable $X$ is defined as

$$
H_{\alpha}(X)=\frac{1}{1-\alpha} \log \sum_{x} p(x)^{\alpha}
$$

for all $\alpha>0, \alpha \neq 1$. (The logarithm is taken to the base $q$.) It is known that $H_{\alpha}(X)$ is decreasing in $\alpha$ and that $\lim _{\alpha \rightarrow 1} H_{\alpha}(X)=H(X)$. We thus have

$$
\begin{aligned}
H(X \mid Y=y) \leq H_{1 / 2}(X \mid Y=y) & =\log \left[\sum_{x} \sqrt{p(x \mid y)}\right]^{2} \\
& =\log [1+(q-1) Z(X \mid Y=y)],
\end{aligned}
$$

where we define $Z(X \mid Y=y)=\frac{1}{q-1} \sum_{x \neq x^{\prime}} \sqrt{p(x \mid y) p\left(x^{\prime} \mid y\right)}$. The desired inequality is obtained by averaging the above relation over $y$ and using the concavity of $t \rightarrow \log (1+(q-1) t)$.

Proof of (4.11). We define two new random variables $S$ and $T$ with $p(x, y, s, t)=p(x) p(y \mid x) p(s, t \mid x)$, where

$$
p(s, t \mid x)=\left\{\begin{array}{ll}
\frac{1}{2(q-1)}, & \text { if } s=x, t \neq x \\
\frac{1}{2(q-1)}, & \text { if } s \neq x, t=x \\
0, & \text { otherwise }
\end{array} .\right.
$$


Note that the conditional probability $p(x, y \mid s, t)$ is defined only if $s \neq t$ and is non-zero only if $x=s$ or $x=t$. Therefore, if we define for $s \neq t$

$$
Z_{s, t}(X \mid Y)=\sum_{y} \sqrt{p_{X Y \mid S T}(s, y \mid s, t) p_{X Y \mid S T}(t, y \mid s, t)}
$$

we have from Proposition 2.8 that

$$
H(X \mid Y, S=s, T=t) \geq\left[2 Z_{s, t}(X \mid Y)\right]^{2}
$$

The proof then follows from the relations

$$
\begin{aligned}
& H(X \mid Y) \geq H(X \mid Y S T) \\
& \geq \sum_{\substack{s, t: \\
s \neq t}} p(s, t)\left[2 Z_{s, t}(X \mid Y)\right]^{2} \\
&= \sum_{\substack{s, t: \\
s \neq t}} p(s, t)\left[2 \sum_{y}\left(\frac{p_{X Y}(s, y) p_{S T \mid X}(s, t \mid s)}{p_{S T}(s, t)}\right)^{1 / 2}\right. \\
&\left.\cdot\left(\frac{p_{X Y}(t, y) p_{S T \mid X}(s, t \mid t)}{p_{S T}(s, t)}\right)^{1 / 2}\right]^{2} \\
& \geq {\left[\sum_{\substack{s, t: \\
s \neq t}} p(s, t) 2 \sum_{y}\left(\frac{p_{X Y}(s, y) p_{S T \mid X}(s, t \mid s)}{p_{S T}(s, t)}\right)^{1 / 2}\right.} \\
&\left.\cdot\left(\frac{p_{X Y}(t, y) p_{S T \mid X}(s, t \mid t)}{p_{S T}(s, t)}\right)^{1 / 2}\right]^{2} \\
&= {\left[\sum_{\substack{s, t: \\
s \neq t}} \sum_{y} \frac{1}{q-1}\left[p_{X Y}(s, y) p_{X Y}(t, y)\right]^{1 / 2}\right]^{2} } \\
& Z(X \mid Y)^{2} .
\end{aligned}
$$

In the above, the second inequality follows from the convexity of the function $x \rightarrow x^{2}$. 


\section{B A Family of Polarizing Transforms}

Here we show that for all $q=|\mathcal{X}|$, the function $f: \mathcal{X}^{2} \rightarrow \mathcal{X}, f\left(x_{1}, x_{2}\right) \rightarrow$ $x_{1}+\pi\left(x_{2}\right)$ with

$$
\pi(x)= \begin{cases}\lfloor q / 2\rfloor, & \text { if } x=0 \\ x-1, & \text { if } 1 \leq x \leq\lfloor q / 2\rfloor \\ x, & \text { otherwise }\end{cases}
$$

is polarizing (see Definition 4.1). That (p.i) and (p.ii) are satisfied readily follows from $\pi$ being a permutation. It remains to show (p.iii), i.e., that for all $2 \leq K \leq q-1$ and $a_{0}<a_{1}<\ldots<a_{K-1}$ in $\mathcal{X}$, the matrix

$$
B_{i j}=a_{i}+\pi\left(a_{j}\right), \quad i, j=0, \ldots, K-1
$$

has at least $K+1$ distinct entries. We will consider two cases:

$K \geq 3$. We will show, by contradiction, that the sets $\left\{B_{i 1}\right\}$ and $\left\{B_{i(K-1)}\right\}$ are not identical, which leads to the claim. For this purpose, note first that $1 \leq a_{1}<a_{K-1}$. Also, since $B_{i 1}=a_{i}+\pi\left(a_{1}\right)$ and $B_{i(K-1)}=a_{i}+\pi\left(a_{K-1}\right)$, it follows that if $\left\{B_{i 1}\right\}=\left\{B_{i(K-1)}\right\}$, then there exists an $L \leq K$ and distinct $i_{1}, \ldots, i_{L} \in\{0,2,3 \ldots, K-1\}$ such that

$$
\begin{aligned}
B_{1(K-1)} & =B_{i_{1} 1} \\
B_{i_{1}(K-1)} & =B_{i_{2} 1} \\
& \vdots \\
B_{i_{L-1}(K-1)} & =B_{i_{L} 1} \\
B_{i_{L}(K-1)} & =B_{11} .
\end{aligned}
$$

This implies

$$
\begin{aligned}
\pi\left(a_{K-1}\right)-\pi\left(a_{1}\right) & =a_{i_{1}}-a_{1} \\
& =a_{i_{2}}-a_{i_{1}} \\
& \vdots \\
& =a_{1}-a_{i_{L}} .
\end{aligned}
$$

Since the terms on the right-hand side above sum to 0 , we have $L[\pi$ $\left.\left(a_{K-1}\right)-\pi\left(a_{0}\right)\right]=0$. As $a_{i_{1}}, \ldots, a_{i_{L}} \neq a_{1}$, this implies that $L$ divides $q$, 
which in turn implies

$$
\max _{i=0, \ldots, K-1}\left(a_{i}-a_{i-1}\right) \leq\lfloor q / 2\rfloor
$$

(where $a_{-1}=a_{K-1}$ ) and thus

$$
a_{K-1}-a_{0} \geq\lfloor q / 2\rfloor .
$$

We therefore have $1 \leq a_{1} \leq\lfloor q / 2\rfloor<a_{K-1}$. It then follows from (4.21) that $a_{i_{1}}-a_{1}=a_{K-1}-a_{1}+1$, i.e., $a_{i_{1}}=a_{K-1}+1$, a contradiction.

$K=2$. Suppose contrary to the claim, that $\left\{B_{00}, B_{10}\right\}=\left\{B_{01}, B_{11}\right\}$. This implies $B_{01}=B_{10}$, i.e.,

$$
a_{1}-a_{0}=\pi\left(a_{0}\right)-\pi\left(a_{1}\right) .
$$

A similar reasoning to the one for the case $K \geq 3$ also yields (4.22). Since $K=2$, it follows that $a_{1}-a_{0}=\lfloor q / 2\rfloor$. On the other hand, it follows from the definition of $\pi$ that

$$
a_{1}-a_{0}=\lfloor q / 2\rfloor \quad \text { implies } \quad \pi\left(a_{0}\right)-\pi\left(a_{1}\right) \neq\lfloor q / 2\rfloor,
$$

contradicting (4.23). This completes the proof.

\section{C An Alternative Proof of Polarization for Prime $q$}

One can prove Theorem 4.3 by first showing that the $Z$ parameters polarize through Arıkan's construction, which by Proposition 4.8 implies the polarization of entropies.

For this purpose, let us first define, for $d=1, \ldots, q-1$, the parameters

$$
Z_{d}(X \mid Y):=\sum_{x} \sum_{y} \sqrt{p(x, y) p(x+d, y)}
$$

It is easy to verify that $Z_{d}(X \mid Y)$ takes values in $[0,1]$. Clearly, $Z(X \mid$ $Y)$ is the mean of $Z_{d}$ 's:

$$
Z(X \mid Y)=\frac{1}{q-1} \sum_{d \neq 0} Z_{d}(X \mid Y)
$$

We also define

$$
Z_{\max }(X \mid Y):=\max _{d \neq 0} Z_{d}(X \mid Y)
$$


We will show that the $Z_{\text {max }}$ 's created by Arıkan's construction converge to 0 or 1 . In order to translate this to a polarization result for entropies, we need $Z_{\max }$ to satisfy

$$
\begin{aligned}
& Z_{\max }(X \mid Y) \approx 1 \text { if and only if } H(X \mid Y) \approx 1 \\
& Z_{\max }(X \mid Y) \approx 0 \text { if and only if } H(X \mid Y) \approx 0 .
\end{aligned}
$$

The second of these relations is evident, since $Z(X \mid Y) \leq Z_{\max }(X \mid$ $Y) \leq(q-1) Z(X \mid Y)$. The following lemma implies that the first relation also holds when $q$ is prime:

Lemma 4.15. For all prime $q$ and $\delta>0$, there exists $\eta(\delta, q)>0$ such that $Z_{\max }(X \mid Y) \geq 1-\eta(\delta, q)$ implies $Z(X \mid Y) \geq 1-\delta$.

Proof. Let $d$ be such that $Z_{d}(X \mid Y)=Z_{\max }(X \mid Y)$. Since $q$ is prime, $\mathcal{X}$ can be written as

$$
\mathcal{X}=\left\{a_{i}: a_{i}=x+i d, i=0, \ldots, q-1\right\}
$$

for all $x \in \mathcal{X}$. Setting $\zeta_{x, x^{\prime}}:=\sum_{y} \sqrt{p(y \mid x) p\left(y \mid x^{\prime}\right)}$ we thus have

$$
Z_{d}(X \mid Y)=\sum_{i=0}^{q-1} \sqrt{p_{X}\left(a_{i}\right) p_{X}\left(a_{i+1}\right)} \cdot \zeta_{a_{i}, a_{i+1}}
$$

It is easily verified that $Z_{d}(X \mid Y)$ is strictly concave in $p_{X Y}$, attaining its maximum when $p_{X}$ is the uniform distribution, and $\zeta_{a_{i}, a_{i+1}}=1$ for all $i$. It then follows that there exists $\nu(\delta)$ such that $Z_{d}(X \mid Y) \geq 1-$ $\eta(\delta)$ implies

(i) $p_{X}(x) \geq 1 / q-\nu(\delta)$ for all $x$,

(ii) $\zeta_{a_{i}, a_{i+1}} \geq 1-\nu(\delta)$ for all $i$,

where $\nu \rightarrow 0$ as $\eta \rightarrow 0$. Now define

$$
\begin{aligned}
& b_{y}=\sqrt{p\left(y \mid a_{i}\right)}-\sqrt{p\left(y \mid a_{i+1}\right)}, \\
& c_{y}=\sqrt{p\left(y \mid a_{i+1}\right)}-\sqrt{p\left(y \mid a_{i+2}\right)} .
\end{aligned}
$$

for all $y \in \mathcal{Y}$. The triangle inequality states that

$$
\left(\sum_{y}\left(b_{y}+c_{y}\right)^{2}\right)^{1 / 2} \leq\left(\sum_{y} b_{y}^{2}\right)^{1 / 2}+\left(\sum_{y} c_{y}^{2}\right)^{1 / 2}
$$


or equivalently, that

$$
\begin{aligned}
\sqrt{1-\zeta_{a_{i}, a_{i+2}}} & \leq \sqrt{1-\zeta_{a_{i}, a_{i+1}}}+\sqrt{1-\zeta_{a_{i+1}, a_{i+2}}} \\
& \leq 2 \sqrt{\nu(\delta)} .
\end{aligned}
$$

Applying the above inequality repeatedly yields

$$
\sqrt{1-\zeta_{x, x^{\prime}}} \leq(q-1) \sqrt{\nu(\delta)}
$$

for all $x, x^{\prime} \in \mathcal{X}$, which implies

$$
\begin{aligned}
Z(X \mid Y) & =\frac{1}{q-1} \sum_{x, x^{\prime}: x \neq x^{\prime}} \sqrt{p(x) p\left(x^{\prime}\right)} \cdot \zeta_{x, x^{\prime}} \\
& \geq[1-q \nu(\delta)]\left[1-(q-1)^{2} \nu(\delta)\right],
\end{aligned}
$$

yielding the claim.

Proposition 4.16. If $\left(X_{1}, Y_{1}\right)$ and $\left(X_{2}, Y_{2}\right)$ are i.i.d., then

$$
\begin{aligned}
Z_{\max }\left(X_{1}+X_{2} \mid Y_{1}^{2}\right) & \leq(q-1)\left(q^{2}-q+1\right) Z_{\max }\left(X_{1} \mid Y_{1}\right) \\
Z_{\max }\left(X_{2} \mid Y_{1}^{2}, X_{1}+X_{2}\right) & =Z_{\max }\left(X_{1} \mid Y_{1}\right)^{2} .
\end{aligned}
$$

Proof. The first claim follows from (4.12):

$$
\begin{aligned}
Z_{\max }\left(X_{1}+X_{2} \mid Y_{1}^{2}\right) & \leq(q-1) Z\left(X_{1}+X_{2} \mid Y_{1}^{2}\right) \\
& \leq(q-1)\left(q^{2}-q+1\right) Z\left(X_{1} \mid Y_{1}\right) \\
& \leq(q-1)\left(q^{2}-q+1\right) Z_{\max }\left(X_{1} \mid Y_{1}\right) .
\end{aligned}
$$

To obtain the second claim we write

$$
\begin{aligned}
Z_{d}\left(X_{2}\right. & \left.\mid Y_{1}^{2}, X_{1}+X_{2}\right) \\
= & \sum_{x_{2}} \sum_{u, y_{1}, y_{2}}\left[p_{X Y}\left(x_{2}, y_{2}\right) p_{X Y}\left(x_{2}+d, y_{2}\right)\right]^{1 / 2} \\
& \cdot\left[p_{X Y}\left(u-x_{2}, y_{1}\right) p_{X Y}\left(u-x_{2}-d, y_{1}\right)\right]^{1 / 2} \\
= & \sum_{x_{2}, y_{2}}\left[p_{X Y}\left(x_{2}, y_{2}\right) p_{X Y}\left(x_{2}+d, y_{2}\right)\right]^{1 / 2} \\
& \cdot \sum_{u, y_{1}}\left[p_{X Y}\left(u-x_{2}, y_{1}\right) p_{X Y}\left(u-x_{2}-d, y_{1}\right)\right]^{1 / 2}
\end{aligned}
$$


Observing that both of the summations above are equal to $Z_{d}\left(X_{1} \mid Y_{1}\right)$, we have $Z_{d}\left(X_{2} \mid Y_{1}, Y_{2}, X_{1}+X_{2}\right)=Z_{d}\left(X_{1} \mid Y_{1}\right)^{2}$. This implies the claim since $t \rightarrow t^{2}$ is increasing for non-negative $t$.

Lemma 4.17. Suppose $B_{1}, B_{2}, \ldots$ are i.i.d., $\{-,+\}$-valued random variables with

$$
P\left(B_{1}=-\right)=P\left(B_{1}=+\right)=\frac{1}{2}
$$

defined on a probability space $(\Omega, \mathcal{F}, P)$. Set $\mathcal{F}_{0}=\{\phi, \Omega\}$ as the trivial $\sigma$-algebra and set $\mathcal{F}_{n}, n \geq 1$ to be the $\sigma$-field generated by $\left(B_{1}, \ldots, B_{n}\right)$.

Suppose further that two stochastic processes $\left\{I_{n}: n \geq 0\right\}$ and $\left\{T_{n}: n \geq 0\right\}$ are defined on this probability space with the following properties:

(i.1) $I_{n}$ takes values in the interval $[0,1]$ and is measurable with respect to $\mathcal{F}_{n}$. That is, $I_{0}$ is a constant, and $I_{n}$ is a function of $B_{1}, \ldots, B_{n}$.

(i.2) $\left\{\left(I_{n}, \mathcal{F}_{n}\right): n \geq 0\right\}$ is a martingale.

(t.1) $T_{n}$ takes values in the interval $[0,1]$ and is measurable with respect to $\mathcal{F}_{n}$.

(t.2) $T_{n+1}=T_{n}^{2}$ when $B_{n+1}=+$.

(i\&t.1) For any $\epsilon>0$ there exists $\delta>0$ such that $I_{n} \in(\epsilon, 1-\epsilon)$ implies $T_{n} \in(\delta, 1-\delta)$.

Then, $I_{\infty}:=\lim _{n \rightarrow \infty} I_{n}$ exists with probability $1, I_{\infty}$ takes values in $\{0,1\}$, and $P\left(I_{\infty}=1\right)=I_{0}$.

Proof. The almost sure convergence of $I_{n}$ to a limit follows from $\left\{I_{n}\right\}$ being a bounded martingale. Once it is known that $I_{\infty}$ is $\{0,1\}$-valued it will then follow from the martingale property that $P\left(I_{\infty}=1\right)=$ $E\left[I_{\infty}\right]=I_{0}$. It thus remains to prove that $I_{\infty}$ is $\{0,1\}$-valued. This in turn is equivalent to showing that for any $\eta>0$,

$$
P\left(I_{\infty} \in(\eta, 1-\eta)\right)=0 .
$$


Since for any $0<\epsilon<\eta$, the event $\left\{I_{\infty} \in(\eta, 1-\eta)\right\}$ is included in the event

$$
J_{\epsilon}:=\left\{\omega: \text { there exists } m \text { such that for all } n \geq m, I_{n} \in(\epsilon, 1-\epsilon)\right\},
$$

and since by property (i\&t.1) there exists $\delta>0$ such that $J_{\epsilon} \subset K_{\delta}$ where

$$
K_{\delta}:=\left\{\omega: \text { there exists } m \text { such that for all } n \geq m, T_{n} \in(\delta, 1-\delta)\right\},
$$

it suffices to prove that $P\left(K_{\delta}\right)=0$ for any $\delta>0$. This is trivially true for $\delta \geq 1 / 2$. Therefore, it suffices to show the claim for $0<\delta<1 / 2$. Given such a $\delta$, find a positive integer $k$ for which $(1-\delta)^{2^{k}}<\delta$. This choice of $k$ guarantees that if a number $x \in[0,1-\delta]$ is squared $k$ times in a row, the result lies in $[0, \delta)$.

For $n \geq 1$ define $E_{n}$ as the event that $B_{n}=B_{n+1}=\cdots=$ $B_{n+k-1}=+$, i.e., $E_{n}$ is the event that there are $k$ consecutive +'s in the sequence $\left\{B_{i}: i \geq 1\right\}$ starting at index $n$. Note that $P\left(E_{n}\right)=2^{-k}>0$, and that $\left\{E_{m k}: m \geq 1\right\}$ is a collection of independent events. The Borel-Cantelli lemma thus lets us conclude that the event

$$
\begin{aligned}
E & =\left\{E_{n} \text { occurs infinitely often }\right\} \\
& =\left\{\omega: \text { for every } m \text { there exists } n \geq m \text { such that } \omega \in E_{n}\right\}
\end{aligned}
$$

has probability 1 , and thus $P\left(K_{\delta}\right)=P\left(K_{\delta} \cap E\right)$. We will now show that $K_{\delta} \cap E$ is empty, from which it will follow that $P\left(K_{\delta}\right)=0$. To that end, suppose $\omega \in K_{\delta} \cap E$. Since $\omega \in K_{\delta}$, there exists $m$ such that $T_{n}(\omega) \in$ $(\delta, 1-\delta)$ whenever $n \geq m$. But since $\omega \in E$ there exists $n_{0} \geq m$ such that $B_{n_{0}+1}=\cdots=B_{n_{0}+k-1}=+$, and thus $T_{n_{0}+k}(\omega)=T_{n_{0}}(\omega)^{2^{k}} \leq(1-$ $\delta)^{2^{k}}<\delta$ which contradicts with $T_{n_{0}+k}(\omega) \in(\delta, 1-\delta)$.

Proof of Theorem 4.3. Let $B_{1}, B_{2}, \ldots$ be an i.i.d. binary process with $\operatorname{Pr}\left[B_{1}=+\right]=1 / 2$. Define $H_{0}, H_{1}, \ldots$ and $Z_{0}, Z_{1}, \ldots$ as in (4.6) and (4.14), respectively. We will show that the conditions of Lemma 4.17 are satisfied if $I_{n}$ and $T_{n}$ are replaced with $H_{n}$ and $Z_{n}$, respectively: That (i.1), (i.2) and (t.1) are satisfied is clear by the definitions of $H_{n}$ and $Z_{n}$, (t.2) is established in Proposition 4.16, and (i\&t.1) follows from Proposition 4.8 and Lemma 4.15. The claim is then a corollary to Lemma 4.17. 


\section{Generalized Constructions}

In the preceding sections, polarization was achieved using a fixed recipe: choose a transform that acts on two random variables, and use it recursively. For prime alphabet sizes, an appropriate choice of mapping was $\left(X_{1}, X_{2}\right) \rightarrow\left(X_{1}+X_{2}, X_{2}\right)$, or equivalently

$$
\left[\begin{array}{ll}
U_{1} & U_{2}
\end{array}\right]=\left[\begin{array}{ll}
X_{1} & X_{2}
\end{array}\right]\left[\begin{array}{ll}
1 & 0 \\
1 & 1
\end{array}\right] .
$$

Some thought reveals that an $n$-fold application of this mapping to a block of $N=2^{n}$ symbols $X_{1}^{N}$ is equivalent to [4]

$$
U_{1}^{N}=X_{1}^{N}\left[\begin{array}{ll}
1 & 0 \\
1 & 1
\end{array}\right]^{\otimes n} B_{n},
$$

where ' $\otimes n$ ' is the $n$th Kronecker power of a matrix, and $B_{n}$ is an $N \times N$ permutation matrix known as the bit-reversal operator. (Recall that the inclusion of the permutation matrix $B_{n}$ is out of notational convenience only.) In this section, we will study generalizations of this method.

Finding transformations that polarize memoryless processes becomes an easy task if one completely disregards complexity issues. 
In fact, almost all invertible binary matrices polarize such processes. This is most easily seen in the following case. Consider an i.i.d. process $\left(X_{1}, Y_{1}\right),\left(X_{2}, Y_{2}\right), \ldots$ where $X_{1}$ is uniformly distributed on $\{0,1\}$, and $Y_{1}$ is the output of a symmetric binary-input memoryless channel with input $X_{1}$. One can think of $X_{1}^{N}$ as codewords obtained through

$$
X_{1}^{N}=U_{1}^{N} G_{N}
$$

where $U_{1}^{N}$ is uniformly distributed over $\{0,1\}^{N}$, and $G_{N}$ is an invertible $\{0,1\}$-matrix. Suppose that $G_{N}$ is chosen through the following procedure: The bottom $R=1-H\left(X_{1} \mid Y_{1}\right)-\epsilon$ fraction of the rows are chosen independently and uniformly at random from $\{0,1\}^{N}$. These rows will be linearly independent with high probability. The remaining $1-R$ fraction of the rows are then chosen in any manner that ensures the invertibility of $G_{N}$. We know from [14, Section 6.2] that with high probability, the code generated by the bottom $R$ fraction of the rows will have exponentially small error probability (in the blocklength) over the channel $X_{1} \rightarrow Y_{1}$. This means, by virtue of Fano's inequality, that $H\left(U_{N(1-R)+1}^{N} \mid Y_{1}^{N} U_{1}^{N(1-R)}\right)$ can be made arbitrarily small as $N$ grows without bound, i.e.,

$$
H\left(U_{i} \mid Y_{1}^{N} U_{1}^{i-1}\right) \rightarrow 0, \quad \text { for all } i>N(1-R) .
$$

It also follows from the above relation and $H\left(U_{1}^{N} \mid Y_{1}^{N}\right) \geq N H\left(X_{1} \mid Y_{1}\right)$ that almost all of the conditional entropies $H\left(U_{i} \mid Y_{1}^{N} U_{1}^{i-1}\right)$ that are not close to 0 must be close to 1 . That is, a typical random matrix generated in this fashion will polarize the underlying process. On the other hand, such matrices will typically have no useful structure, and thus one may not be able to find low-complexity algorithms to decode the generated codes. The decoding complexity of such codes will typically be exponential in the blocklength.

The above argument can be stated more generally. Observe that in a channel code with messages $U_{1}^{N R}$, codewords $X_{1}^{N}$, channel outputs $Y_{1}^{N}$ and small block error probability, the entropy

$$
H\left(U_{1}^{N R} \mid Y_{1}^{N}\right)=\sum_{i=1}^{N R} H\left(U_{i} \mid Y_{1}^{N} U_{1}^{i-1}\right)
$$


is also small. That is, almost all terms on the right-hand side of the above are close to 0 . Hence, any good code can be thought of as one that polarizes the resulting process of channel inputs and outputs. A similar statement also holds for good source codes. Polarization, if defined as the creation of extremal entropies from mediocre ones, is then not peculiar to polar codes, but is common to all good codes. The main virtue of polar codes is not that they polarize processes, but that they do so in a recursive fashion. It is this recursive structure that enables their good performance under low-complexity successive cancellation decoding.

\subsection{Recursive Transforms}

In view of the discussion above, it is reasonable to restrict the search for methods of polarization to recursive ones. We will focus on the easiest way of obtaining such transforms: replacing the matrix $\left[\begin{array}{ll}1 & 0 \\ 1 & 1\end{array}\right]$ in the original construction with another square matrix, possibly of a larger size. More precisely, we will assume that the process $\left(X_{1}, Y_{1}\right),\left(X_{2}, Y_{2}\right), \ldots$ is i.i.d. and $X_{1}$ takes values over a finite field $\mathbb{F}_{q}$ of prime size, and we will study transforms of the form

$$
U_{1}^{N}=X_{1}^{N} G^{\otimes n} B_{n}
$$

where $N=\ell^{n}$, matrix multiplication is over $\mathbb{F}_{q}$, and $G$ is an $\ell \times \ell$ $\mathbb{F}_{q}$-matrix with $\ell \geq 2$. The $N \times N$ permutation matrix $B_{n}$ is defined analogously to the bit-reversal operation in the original construction: It corresponds to the permutation $f(i)=r_{\ell}(i-1)+1, i=1, \ldots, N$, where $r_{\ell}(i)=j$ for $i$ and $j$ with $\ell$-ary expansions $b_{n} \ldots b_{1}$ and $b_{1} \ldots b_{n}$, respectively.

In addition to their low encoding and decoding complexity, codes based on recursive transforms are also amenable to error analysis. As in Arıkan's original construction, the large blocklength behavior of recursive transforms is dictated by certain properties of the basic transform $G$, and therefore several useful conclusions can be drawn simply by establishing these properties. We will in particular study the following questions: (i) What choices of $G$ yield polarizing transforms? (ii) What 
is the error probability behavior of such codes? We will see that the answers to both questions are fairly simple.

\subsection{Polarizing Matrices}

We will say that a matrix $G$ is a polarizing matrix if it is invertible and a recursive application of it as in (5.1) yields

$$
\begin{aligned}
& \lim _{n \rightarrow \infty} \frac{1}{N}\left|\left\{i: H\left(U_{i} \mid Y_{1}^{N} U_{1}^{i-1}\right)>1-\epsilon\right\}\right|=H\left(X_{1} \mid Y_{1}\right) \\
& \lim _{n \rightarrow \infty} \frac{1}{N}\left|\left\{i: H\left(U_{i} \mid Y_{1}^{N} U_{1}^{i-1}\right)<\epsilon\right\}\right|=1-H\left(X_{1} \mid Y_{1}\right)
\end{aligned}
$$

for all $\epsilon>0$ and all i.i.d. processes $\left(X_{1}, Y_{1}\right),\left(X_{2}, Y_{2}\right), \ldots$, exactly as in the original construction. It is a direct consequence of Hall's theorem [10, Theorem 16.4] that given an invertible matrix $G$, there exists a permutation matrix $P$ such that $G P$ has non-zero diagonal entries. We will therefore assume throughout, and without loss of generality, that all of the diagonal entries of $G$ are non-zero (for otherwise it can be reduced to this form by permuting its columns). Recall that a necessary condition for polarization is that the 'entropy paths' generated along the recursion always fork until they converge to 0 or 1 (see Figure 4.1), i.e., that at least one of the created entropies at each step be different from the others. This requirement is met by a large class of matrices:

Lemma 5.1. Let $S_{1}^{\ell}=X_{1}^{\ell} G$ for some invertible matrix $G$.

(i) If $G$ is upper-triangular, then $H\left(S_{i} \mid Y_{1}^{\ell} S_{1}^{i-1}\right)=H\left(X_{1} \mid Y_{1}\right)$ for all $i=1, \ldots, \ell$.

(ii) If $G$ is not upper-triangular, then for every $\epsilon>0$ there exists $\delta(\epsilon)>0$ and $i \in\{1, \ldots, \ell\}$ such that

$$
H\left(X_{1} \mid Y_{1}\right) \in(\epsilon, 1-\epsilon)
$$

implies

$$
H\left(S_{i} \mid Y_{1}^{\ell} S_{1}^{i-1}\right)-H\left(X_{1} \mid Y_{1}\right)>\delta(\epsilon)
$$


Proof. Let $g_{i j}$ denote the $(i, j)$ th entry of $G$. If $G$ is upper-triangular, $H\left(S_{i} \mid Y_{1}^{\ell} S_{1}^{i-1}\right)$ can be written as

$$
\begin{aligned}
& H\left(S_{i} \mid Y_{1}^{\ell} S_{1}^{i-1}\right) \\
& \quad=H\left(\sum_{j=1}^{i} g_{j i} X_{j} \mid Y_{1}^{\ell}, g_{11} X_{1}, g_{12} X_{1}+g_{22} X_{2}, \ldots, \sum_{j=1}^{i-1} g_{j i} X_{j}\right) .
\end{aligned}
$$

Since $G$ is invertible, its first $i-1$ columns are linearly independent, and therefore the above can be rewritten as

$$
H\left(S_{i} \mid Y_{1}^{\ell} S_{1}^{i-1}\right)=H\left(\sum_{j=1}^{i} g_{j i} X_{j} \mid Y_{1}^{\ell}, X_{1}^{i-1}\right)=H\left(X_{i} \mid Y_{i}\right),
$$

proving (i). If on the other hand $G$ is not upper-triangular, then let $i \in\{1, \ldots, \ell\}$ be the smallest index for which the $i$ th column of $G$ has at least two non-zero entries $g_{k i}$ and $g_{l i}$ below and including the diagonal. Such an $i$ always exists. Since $\left(X_{1}, Y_{1}\right), \ldots,\left(X_{\ell}, Y_{\ell}\right)$ are independent, and since summing independent random variables increases entropy, we have

$$
\begin{aligned}
H\left(S_{i} \mid Y_{1}^{\ell} S_{1}^{i-1}\right) & =H\left(\sum_{j=1}^{\ell} g_{j i} X_{j} \mid Y_{1}^{\ell} S_{1}^{i-1}\right) \\
& \geq H\left(g_{k i} X_{k}+g_{l i} X_{l} \mid Y_{1}^{\ell} S_{1}^{i-1}\right) \\
& =H\left(g_{k i} X_{k}+g_{l i} X_{l} \mid Y_{k} Y_{l}\right),
\end{aligned}
$$

where the second equality is due to the definition of $i$. Observe now that the last entropy term can be written as $H\left(\tilde{X}_{k}+\tilde{X}_{l} \mid Y_{k}, Y_{l}\right)$, where $\tilde{X}_{k}$ and $\tilde{X}_{l}$ are appropriately permuted versions of $X_{k}$ and $X_{l}$, respectively. The claim then follows from Lemma 4.2.

The following polarization result can be proven as a corollary to the above lemma, using the standard martingale argument. (See the proof of Theorem 2.3.)

Theorem 5.2. For all prime $q$, an invertible $\mathbb{F}_{q}$-matrix is polarizing unless it is upper-triangular. 
The above theorem says that the class of polarizing matrices is large. One may therefore hope to find, in this large class, matrices that yield better codes than the original polar codes in terms of their error probabilities. We study this problem next.

\subsection{Rate of Polarization}

Recall that for constructions based on combining two random variables at a time, convergence of the Bhattacharyya parameters was exponential roughly in the square root of the blocklength, i.e., we had

$$
\lim _{n \rightarrow \infty} \frac{1}{N}\left|\left\{i: Z\left(U_{i} \mid Y_{1}^{N} U_{1}^{i-1}\right)<2^{-N^{\beta}}\right\}\right|=1-H\left(X_{1} \mid Y_{1}\right)
$$

for all $\beta<1 / 2$. Let us recall the reason behind this behavior: Throughout the recursion, a Bhattacharyya parameter is (roughly) squared in approximately half of the recursions, and is unaffected (i.e., raised to power 1) in the remaining recursions. Since each recursion also doubles the blocklength, a simple calculation shows that the exponent of a typical Bhattacharyya parameter $Z$ is roughly $\frac{1}{2} \log _{2} 2+\frac{1}{2} \log _{2} 1=\frac{1}{2}$, i.e., $Z \approx 2^{-N^{1 / 2}}$. (Note that we still need to prove these statements, as they neglect the multiplicative constants appearing in the bounds on the Bhattacharyya parameters.) It is also intuitively evident that the same argument can be made for any recursive construction: If an $\ell \times \ell$ matrix $G$ creates $\ell$ Bhattacharyya parameters that are roughly equal to $Z\left(X_{1} \mid Y_{1}\right)^{a_{1}}, \ldots, Z\left(X_{1} \mid Y_{1}\right)^{a_{\ell}}$, then after many recursions the exponent of a typical Bhattacharyya parameter would be given by $\mathrm{E}=\frac{1}{\ell} \log _{\ell} a_{1}+\ldots+\frac{1}{\ell} \log _{\ell} a_{\ell}$, i.e., $Z \approx 2^{-N^{\mathrm{E}}}$. That is, the large scale behavior of the Bhattacharyya parameters is determined by their onestep evolution. It thus suffices to study how the underlying matrix $G$ transforms the Bhattacharyya parameters in a single recursion. It turns out that this transformation is determined largely by the partial distances of $G^{-1}$ :

Definition 5.1. Let $G$ be an $\ell \times \ell$ matrix with rows $g_{1}, \ldots, g_{\ell} \in \mathbb{F}_{q}^{\ell}$. The partial distances $D_{1}, \ldots, D_{\ell}$ of $G$ are defined as

$$
D_{i}=\mathrm{d}_{H}\left(\left\langle g_{i}\right\rangle,\left\langle g_{i+1}, \ldots, g_{\ell}\right\rangle\right)
$$




\section{Generalized Constructions}

where $\langle a\rangle$ denotes the vector space spanned by $a$, and

$$
\mathrm{d}_{H}(\langle a\rangle,\langle b\rangle):=\min _{\substack{x \in\langle a\rangle, y \in\langle b\rangle \\ x \neq 0}} \mathrm{~d}_{H}(x, y)
$$

where $\mathrm{d}_{H}(x, y)$ denotes the Hamming distance between vectors $x$ and $y$.

Proposition 5.3. Let $S_{1}^{\ell}=X_{1}^{\ell} G$, and let $D_{1}, \ldots, D_{\ell}$ be the partial distances of $G^{-1}$. We have

$$
Z\left(S_{i} \mid Y_{1}^{\ell} S_{1}^{i-1}\right) \leq q^{3 \ell} Z\left(X_{1} \mid Y_{1}\right)^{D_{i}}, \quad i=1, \ldots, \ell .
$$

Proof. Note first that

$$
p_{S_{1}^{i} Y_{1}^{\ell}}\left(s_{1}^{i}, y_{1}^{\ell}\right)=\sum_{s_{i+1}^{\ell}} p_{S_{1}^{\ell} Y_{1}^{\ell}}\left(s_{1}^{\ell}, y_{1}^{\ell}\right)=\sum_{s_{i+1}^{\ell}} \prod_{i=1}^{\ell} p_{X Y}\left(\left[s_{1}^{\ell} G^{-1}\right]_{i}, y_{i}\right) .
$$

We have

$$
\begin{aligned}
Z\left(S_{i} \mid Y_{1}^{\ell} S_{1}^{i-1}\right) & \frac{1}{q-1} \sum_{s \neq s^{\prime}} \sum_{y_{1}^{\ell}, s_{1}^{i-1}}\left[p_{S_{1}^{i} Y_{1}^{\ell}}\left(\left(s_{1}^{i-1}, s\right), y_{1}^{\ell}\right) p_{S_{1}^{i} Y_{1}^{\ell}}\left(\left(s_{1}^{i-1}, s^{\prime}\right), y_{1}^{\ell}\right)\right]^{1 / 2} \\
= & \frac{1}{q-1} \sum_{s \neq s^{\prime}} \sum_{y_{1}^{\ell}, s_{1}^{i-1}}\left[\sum_{v_{i+1}^{\ell}} \prod_{i} p_{X Y}\left(\left[\left(s_{1}^{i-1}, s, v_{i+1}^{\ell}\right) G^{-1}\right]_{i}, y_{i}\right)\right. \\
& \left.\cdot \sum_{w_{i+1}^{\ell}} \prod_{i} p_{X Y}\left(\left[\left(s_{1}^{i-1}, s^{\prime}, w_{i+1}^{\ell}\right) G^{-1}\right]_{i}, y_{i}\right)\right]^{1 / 2} \\
\leq & \frac{1}{q-1} \sum_{s \neq s^{\prime}} \sum_{y_{1}^{\ell}, s_{1}^{i-1}} \sum_{v_{i+1}^{\ell}, w_{i+1}^{\ell}}\left[\prod_{i} p_{X Y}\left(\left[\left(s_{1}^{i-1}, s, v_{i+1}^{\ell}\right) G^{-1}\right]_{i}, y_{i}\right)\right. \\
& \left.\cdot p_{X Y}\left(\left[\left(s_{1}^{i-1}, s^{\prime}, w_{i+1}^{\ell}\right) G^{-1}\right]_{i}, y_{i}\right)\right]^{1 / 2} \cdot
\end{aligned}
$$


Observe that for all $s_{1}^{i-1}, v_{i+1}^{\ell}$, and $w_{i+1}^{\ell}$ we have

$$
\mathrm{d}_{H}\left(\left(s_{1}^{i-1}, s, v_{i+1}^{\ell}\right) G^{-1},\left(s_{1}^{i-1}, s^{\prime}, w_{i+1}^{\ell}\right) G^{-1}\right) \geq D_{i},
$$

and therefore

$$
\begin{aligned}
\sum_{y_{1}^{\ell}} & {\left[\prod_{i} p_{X Y}\left(\left[\left(s_{1}^{i-1}, s, v_{i+1}^{\ell}\right) G^{-1}\right]_{i}, y_{i}\right)\right.} \\
& \left.\cdot p_{X Y}\left(\left[\left(s_{1}^{i-1}, s^{\prime}, w_{i+1}^{\ell}\right) G^{-1}\right]_{i}, y_{i}\right)\right]^{1 / 2} \leq\left[(q-1) Z\left(X_{1} \mid Y_{1}\right)\right]^{D_{i}} .
\end{aligned}
$$

Combining this relation with (5.3) yields the claim.

We can now characterize the error probability behavior of general recursive polar codes. For this purpose, we first define the exponent $\mathrm{E}(G)$ of a matrix $G$, through the partial distances $D_{1}, \ldots, D_{\ell}$ of $G^{-1}$ :

$$
\mathrm{E}(G):=\frac{1}{\ell} \sum_{i=1}^{\ell} \log _{\ell} D_{i} .
$$

Theorem 5.4. Let $G$ be an $\ell \times \ell$ polarizing matrix and $U_{1}^{N}$ be defined as in (5.1). Then,

$$
\lim _{n \rightarrow \infty} \frac{1}{N}\left|\left\{i: Z\left(U_{i} \mid Y_{1}^{N} U_{1}^{i-1}\right)<2^{-N^{\beta}}\right\}\right|=1-H\left(X_{1} \mid Y_{1}\right)
$$

for all $\beta<\mathrm{E}(G)$.

We defer the proof of Theorem 5.4 to Section 5.4. This result yields an asymptotic upper bound on the error probability of polar source and channel codes. That is, we asymptotically have $P_{\mathrm{e}} \leq 2^{-N^{\beta}}$ for all $\beta<$ $\mathrm{E}(G)$. Note that this bound holds for all polar source and channel codes whose rates are respectively above source entropy and below channel capacity. On the other hand, it is desirable to establish more refined bounds that reflect the dependence of error probability on the code rate. 
This can be done by augmenting the large deviations technique in the proof of Theorem 5.4 by a central limit theorem type argument:

Theorem 5.5 $[20,46]$. Let $\mathrm{V}(G)$ be the variance of $\log D_{1}, \ldots, \log _{\ell} D_{\ell}$, that is

$$
\mathrm{V}(G)=\frac{1}{\ell} \sum_{i=1}^{\ell}\left(\log _{\ell} D_{i}-\mathrm{E}(G)\right)^{2}
$$

and let $Q(t):=\frac{1}{\sqrt{2 \pi}} \int_{t}^{\infty} e^{-\tau^{2} / 2} d \tau$. We have for $R<1-H\left(X_{1} \mid Y_{1}\right)$

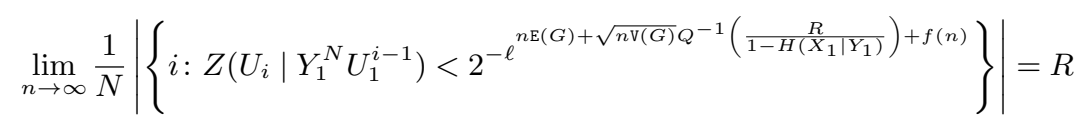

for all $f(n)=o(n)$.

Proof. See [19].

Observe that this result yields an upper bound on the error probability of polar channel codes of rate $R$ and source codes of rate $1-R$.

\subsubsection{Bounds on the Rate of Polarization}

The importance of Proposition 5.3 and Theorem 5.4 is in identifying through $\mathrm{E}(G)$ the exponential dependence between the error probability and the blocklength. This significantly simplifies the search for good recursive constructions since $\mathrm{E}(G)$ is an easy-to-calculate algebraic quantity. One can also use the existing results on the minimum distance of codes to find useful bounds on the best possible $\mathrm{E}(G)$ for a given size, i.e., on

$$
\mathrm{E}_{\ell}:=\max _{G \in \mathbb{F}_{q}^{\ell \times \ell}} \mathrm{E}(G) .
$$

It is useful to note that recursive constructions may not be of much practical value for large values of $\ell$ : It can indeed be verified easily that the decoding complexity of codes based on a general $\ell \times \ell$ recursion is $O\left(q^{\ell} N \log N\right)$. We can therefore restrict our attention to small $\ell$, for which one can either exactly compute or bound $\mathrm{E}_{\ell}$. Conveniently, even the simplest bounding techniques provide useful information at small 
sizes. The following upper and lower bounds on the partial distances based on sphere packing and Gilbert-Varshamov type constructions, respectively — were given in [26] for the binary case:

\section{Proposition 5.6.}

$$
\frac{1}{\ell} \sum_{i=1}^{\ell} \log _{\ell} \tilde{D}_{i} \leq \mathrm{E}_{\ell} \leq \frac{1}{\ell} \sum_{i=1}^{\ell} \log _{\ell} \hat{D}_{i},
$$

where

$$
\hat{D}_{i}=\max \left\{D: \sum_{j=0}^{\left\lfloor\frac{D-1}{2}\right\rfloor}\left(\begin{array}{l}
\ell \\
j
\end{array}\right) \leq q^{i-1}\right\}
$$

and

$$
\tilde{D}_{i}=\max \left\{D: \sum_{j=0}^{D-1}\left(\begin{array}{l}
\ell \\
j
\end{array}\right)<q^{i}\right\}
$$

An improved version of these bounds, along with the exponents of a BCH code-based construction (both given in [26]) are plotted for $q=2$ in Figure 5.1. These results are of a somewhat negative nature, as they show that the original exponent $1 / 2$ of Arıkan's construction cannot be improved at small recursion sizes. It was in fact shown in [26] that $E_{\ell} \leq 1 / 2$ for $\ell<15$, and that $E_{16} \approx 0.51$. Therefore in the binary case, generalized constructions may not be appropriate for achieving substantial gains in error probability without significant sacrifices in complexity. Nevertheless, it follows from the above bounds that one can attain 'almost exponential' error probability decay with the blocklength if the size of the recursion is sufficiently large:

Proposition $5.7([26])$. For all prime $q, \lim _{\ell \rightarrow \infty} E_{\ell}=1$.

The case for generalized constructions is stronger in non-binary settings. This is due to the fact that for a fixed matrix size, larger alphabet sizes allow for better separation (in the Hamming distance) between the rows of a matrix, yielding better exponents at any fixed $\ell$. A simple evidence of this is given in the following result. 


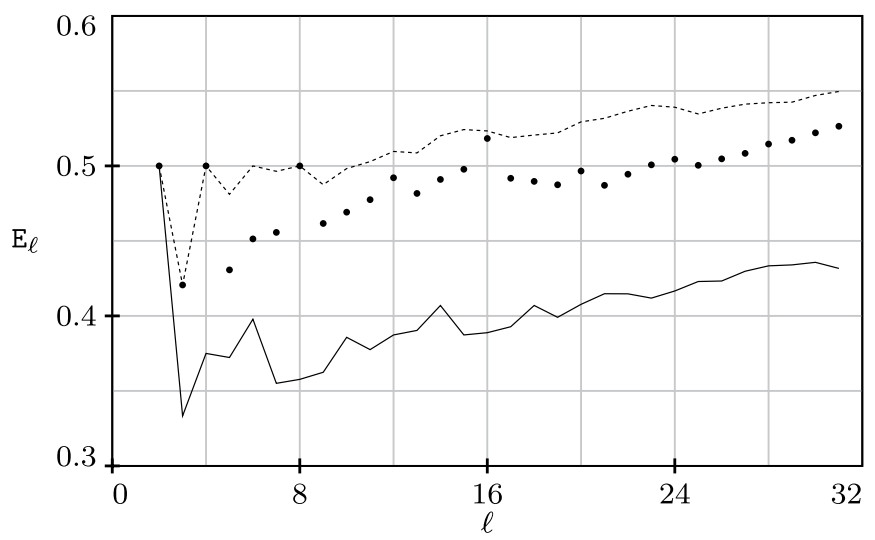

Fig. 5.1 The solid and the dashed curves represent lower and upper bounds on $\mathrm{E}_{\ell}$ (in the binary case), respectively. The dots show the exponents of a $\mathrm{BCH}$ code-based construction (see [26]).

Theorem 5.8. For $\ell \leq q, E_{\ell}=\frac{1}{\ell} \log _{\ell}(\ell !)$.

Proof. Observe first that $D_{i} \leq i$ for any invertible matrix. To see this, note that the invertibility of a matrix $G$ with rows $g_{1}, \ldots, g_{\ell}$ implies that $g_{i+1}, \ldots, g_{\ell}$ have $\ell-i$ linearly independent columns, and thus span $\mathbb{F}_{q}^{\ell-i}$ at the locations corresponding to these columns. Therefore, $g_{i}$ can at most be at a distance $i$ from $\left\langle g_{i+1}, \ldots, g_{\ell}\right\rangle$.

To prove the claim, we only need to find a matrix with $D_{i}=i$. To that end, let $\omega$ be an arbitrary element of $\mathbb{F}_{q}$ other than the identity, and let $G$ be the matrix with rows

$$
g_{i}=\left[1, \omega^{i}, \omega^{2 i}, \ldots, \omega^{(\ell-1) i}\right]
$$

That is, $G$ is the generator matrix of a Reed-Solomon code of rate 1 . It is known that the minimum distance of the code $\left\langle g_{i}, \ldots, g_{\ell}\right\rangle$ is $i[31$, Section 10.2], and therefore

$$
D_{i}=\mathrm{d}_{H}\left(\left\langle g_{i}\right\rangle,\left\langle g_{i+1}, \ldots, g_{\ell}\right\rangle\right) \geq i .
$$

The above theorem implies that for $q \geq 5$, we have $E_{2}=0.5, E_{3} \approx$ $0.54, E_{4} \approx 0.57$, and $E_{5} \approx 0.59$. Compare these with the upper bounds given in Figure 5.1 for the binary case. 


\subsection{Proof of Theorem 5.4}

We will not provide the proof in full, since it is an almost identical reproduction of the proof of Theorem 2.5 once we obtain the following result.

Lemma 5.9. Let $B_{1}, B_{2}, \ldots$ be an i.i.d. process where $B_{1}$ is uniformly distributed over $\{1,2, \ldots, \ell\}$. Also let $Z_{0}, Z_{1}, \ldots$ be a $[0,1]$-valued random process where $Z_{0}$ is constant and

$$
Z_{n+1} \leq K Z_{n}^{D_{i}}, \quad \text { whenever } B_{n}=i
$$

for some $K>0$ and $2 \leq D_{1} \leq \ell$ and $1 \leq D_{2}, \ldots, D_{\ell} \leq \ell$. Suppose also that $Z_{n}$ converges almost surely to a $\{0,1\}$-valued random variable $Z_{\infty}$ with $\operatorname{Pr}\left[Z_{\infty}=0\right]=z$. Then, for any $\beta<E$ where

$$
E=\frac{1}{\ell} \sum_{i} \log _{\ell} D_{i}
$$

we have

$$
\lim _{n \rightarrow \infty} \operatorname{Pr}\left[Z_{n} \leq 2^{-\ell^{\beta n}}\right]=z
$$

Remark 5.1. Note that the definition of the process $Z_{0}, Z_{1}, \ldots$ reflects the transformation of Bhattacharyya parameters in a single recursion (5.2): All partial distances $D_{1}, \ldots, D_{\ell}$ of a polarizing matrix are $\geq 1$ (since the matrix is invertible), with at least one partial distance $\geq 2$ (since the matrix is not upper-triangular).

This result was originally proven for $\ell=2$ by Arıkan and Telatar in [9]. We will provide the general proof in full for completeness, although it is a straightforward extension of the bounding technique given in [9]. As the technique is slightly intricate, it is useful to briefly explain the ideas contained in it: note first that for $K \leq 1$ the result is a simple corollary to the weak law of large numbers: In a sufficiently long sequence $B_{1}, \ldots, B_{n}$, each exponent $D_{i}$ appears nearly $n / \ell$ times with high probability, and thus a typical $Z_{n}$ is less than

$$
Z_{0}^{\prod_{i} D_{i}^{n / \ell}}=\left(1 / Z_{0}\right)^{-\ell^{n E}} \text {. }
$$


It can easily be seen that this method does not yield a useful bound when $K>1$. The proof given below is instead based on the following observations: Whenever $Z_{n}$ converges to zero, there must be a finite point $n_{0}$ for which the sequence $Z_{n}, n>n_{0}$ stays below a given positive threshold $\epsilon$ (Lemma 5.11). This threshold can be chosen sufficiently small so that if $Z_{n} \leq \epsilon$, then $K Z_{n}^{d}$ is approximately the same as $Z_{n}^{d}$ if $d>1$, i.e., multiplying $Z_{n}$ with $K$ has negligible effect compared with exponentiating it. Once this is established, one can again appeal to the law large numbers as in the case $K \leq 1$ to obtain the result.

Lemma 5.10. Let $a_{0}, a_{1}, \ldots$ be a sequence of numbers satisfying

$$
a_{i+1}=b_{i+1} a_{i}+K, \quad i=0,1, \ldots
$$

where $K>0$ and $b_{i} \geq 1$ for all $i$. Then,

$$
a_{n} \leq\left(a_{0}+K n\right) \prod_{i=1}^{n} b_{i} .
$$

Proof. A straightforward computation shows that

$$
a_{n}=a_{0} \prod_{i=1}^{n} b_{i}+K \sum_{i=1}^{n} \prod_{j>i} b_{j}
$$

from which the claim follows trivially.

Lemma 5.11. For every $\epsilon>0$, there exists an $m(\epsilon)$ such that

$$
\operatorname{Pr}\left[Z_{n} \leq 1 / K^{\ell+1} \text { for all } n \geq m(\epsilon)\right]>z-\epsilon .
$$

Proof. Let $\Omega=\left\{\omega: Z_{n}(\omega) \rightarrow 0\right\}$, and note that $\operatorname{Pr}[\Omega]=z$. Also observe that since $Z_{n}$ is non-negative, $\Omega$ can be written as

$$
\begin{aligned}
\Omega= & \left\{\omega: \text { for all } k \geq 1 \text { there exists } n_{0}(\omega)\right. \\
& \text { such that } \left.Z_{n}(\omega)<1 / k \text { for all } n \geq n_{0}(\omega)\right\} \\
= & \bigcap_{k \geq 1} \bigcup_{n_{0} \geq 0} A_{n_{0}, k},
\end{aligned}
$$


where $A_{n_{0}, k}=\left\{\omega: Z_{n}(\omega)<1 / k\right.$ for all $\left.n \geq n_{0}\right\}$. (Note that $n_{0}$ in the definition of $A_{n_{0}, k}$ is independent of $\omega$.) Since the sets $A_{n_{0}, k}$ are increasing in $n_{0}$, for all $\epsilon>0$ there exists an $m(\epsilon)$ for which $\operatorname{Pr}\left[A_{m(\epsilon), k}\right]>$ $\operatorname{Pr}\left[\cup_{n_{0} \geq 0} A_{n_{0}, k}\right]-\epsilon$, and thus taking $k=K^{\ell+1}$ we have

$$
\operatorname{Pr}\left[A_{m(\epsilon), K^{\ell+1}}\right]>\operatorname{Pr}\left[\cup_{n_{0} \geq 0} A_{n_{0}, K^{\ell+1}}\right]-\epsilon \geq \operatorname{Pr}[\Omega]-\epsilon,
$$

yielding the claim.

Lemma 5.12. For all $\epsilon>0$, there exists an $n(\epsilon)$ such that

$$
\operatorname{Pr}\left[\log _{K} Z_{n}<-n / 4 \ell\right]>z-\epsilon
$$

for all $n \geq n(\epsilon)$.

Proof. Given $\epsilon>0$, choose $m$ and $A_{m, K^{\ell+1}}$ as in the proof Lemma 5.11. Observe that inside the set $A_{m, K^{\ell+1}}$ we have, conditioned on $B_{n}=i$,

$$
\begin{aligned}
Z_{n+1} & \leq K Z_{n}^{D_{i}} \\
& \leq K^{1-\left(D_{i}-1\right)(\ell+1)} Z_{n} \\
& \leq \begin{cases}K^{-\ell} Z_{n}, & \text { if } B_{n}=1 \\
K Z_{n}, & \text { if } B_{n}=2, \ldots, \ell\end{cases}
\end{aligned}
$$

or equivalently

$$
\begin{aligned}
& \log _{K} Z_{n+1} \leq \log _{K} Z_{n}-\ell, \quad \text { if } B_{n}=1 \\
& \log _{K} Z_{n+1} \leq \log _{K} Z_{n}+1, \quad \text { if } B_{n}=2, \ldots, \ell .
\end{aligned}
$$

This implies that inside the set $A_{m, K^{\ell+1}}$

$$
\log _{K} Z_{n} \leq \log _{K} Z_{m}+(n-m)(1-\alpha(\ell+1))
$$

where $\alpha$ is the fraction of 1 's in the sequence $B_{m}, \ldots, B_{n}$. Let $T_{m, \alpha}^{n}$ denote the event that the sequence $B_{m}, \ldots, B_{n}$ contains at least an $\alpha$ fraction of each letter $k \in\{1, \ldots, \ell\}$. Now choose $n_{0} \geq 2 m$ such that $\operatorname{Pr}\left[T_{m, \alpha}^{n}\right]>1-\epsilon$ for all $n \geq n_{0}$ with $\alpha=(2 \ell+1) /[(2 \ell+2) \ell]$. 
Note that such an $n_{0}$ exists since $\alpha<1 / \ell$. Then we have inside the set $A_{m, K^{\ell+1}} \cap T_{m, \alpha}^{n}$

$$
\begin{aligned}
\log _{K} Z_{n} & \leq \log _{K} Z_{m}-\frac{n}{2}(1-\alpha(\ell+1)) \\
& \leq-n / 4 \ell
\end{aligned}
$$

Observing that $\operatorname{Pr}\left[A_{m, K^{\ell+1}} \cap T_{m, \alpha}^{n}\right] \geq z-2 \epsilon$ yields the claim.

Proof of Lemma 5.9. We only need to prove the claim for $K>1$. Given $\epsilon>0$, choose $\alpha<1 / \ell$ and $\gamma<1$ such that $\alpha \gamma \ell>1-\epsilon$. Also let $n$ be sufficiently large so that $n_{1}:=\log _{\ell}(2 n K) 8 K / E \alpha n_{2}:=n_{1} / 8 \ell K$ satisfy

(i) $n_{1}>\max \left(n_{0}, 8 \ell\right)$, where $n_{0}$ is as in Lemma 5.12,

(ii) $\operatorname{Pr}\left[T_{n_{1}, \alpha}^{n_{1}+n_{2}}\right]>1-\epsilon$, where $T_{n_{1}, \alpha}^{n_{1}+n_{2}}$ is defined as in the proof of Lemma 5.12,

(iii) $\operatorname{Pr}\left[T_{n_{1}+n_{2}, \alpha}^{n}\right]>1-\epsilon$, and

(iv) $n-\left(n_{1}+n_{2}\right) \geq \gamma n$.

Conditions (i)-(iii) imply that the probability of the set

$$
A=\left\{\log _{K} Z_{n_{1}} \leq-n_{1} / 4 \ell\right\} \cap T_{n_{1}, \alpha}^{n_{2}} \cap T_{n_{1}+n_{2}, \alpha}^{n}
$$

is at least $z-3 \epsilon$. Observe also that the process $L_{n}=\log _{K} Z_{n}$ satisfies

$$
L_{n+1} \leq D_{i} L_{n}+K \quad \text { if } B_{n}=i .
$$

Since inside the set $A$ we have $B_{n}=i$ for at least an $\alpha$ fraction of $B_{n}$, it follows from Lemma 5.10 that

$$
\begin{aligned}
L_{n_{1}+n_{2}} & \leq\left(-n_{1} / 4 \ell+n_{2} K\right) \prod_{m=n_{1}}^{n_{1}+n_{2}} D_{B_{m}} \\
& \leq-\prod_{m=n_{1}}^{n_{1}+n_{2}} D_{B_{m}} \\
& \leq-\prod_{i=1}^{\ell} D_{i}^{\alpha n_{2}} \\
& =-\ell^{E \ell \alpha n_{2}} .
\end{aligned}
$$


Similarly bounding $L_{n}$ we obtain

$$
\begin{aligned}
L_{n} & \leq\left(L_{n_{2}}+\left[n-n_{1}-n_{2}\right] K\right) \prod_{m=n_{1}+n_{2}}^{n} D_{B_{m}} \\
& \leq\left(-\ell^{E \ell \alpha n_{2}}+n K\right) \prod_{m=n_{1}+n_{2}}^{n} D_{B_{m}} \\
& \leq\left(-\ell^{E \alpha n_{1} / 8 K}+n K\right) \prod_{m=n_{1}+n_{2}}^{n} D_{B_{m}} \\
& \leq\left(-\ell^{E \alpha n_{1} / 8 K} / 2\right) \prod_{m=n_{1}+n_{2}}^{n} D_{B_{m}} \\
& \leq-\prod_{m=n_{1}+n_{2}}^{n} D_{B_{m}} \\
& \leq-\prod_{i=1}^{\ell} D_{i}^{\alpha\left(n-n_{1}-n_{2}\right)} \\
& =-\ell^{E \ell \alpha\left(n-n_{1}-n_{2}\right)} \\
& \leq-\ell^{E \ell \alpha \gamma n} \\
& \leq-\ell^{E n(1-\epsilon)}
\end{aligned}
$$

which implies that with probability at least $z-3 \epsilon$

$$
Z_{n} \leq K^{-\ell^{(1-\epsilon) E n}}=2^{-\ell^{\left[(1-\epsilon) E-\log _{\ell}\left(\log _{2} K\right) / n\right] n},}
$$

yielding the claim. 


\section{Joint Polarization of Multiple Processes}

We have by now established that all discrete memoryless stationary (that is, i.i.d.) processes can be polarized by a large class of recursive procedures. These procedures yield low-complexity point-to-point channel codes as well as source codes that achieve optimal rates, i.e., symmetric capacity and source entropy, respectively. Our aim in this section is to apply the principles developed so far in order to obtain joint polarization results for multiple sequences. In particular, we will consider i.i.d. processes of the form $\left(W_{1}, X_{1}, Y_{1}\right),\left(W_{2}, X_{2}, Y_{2}\right), \ldots$ where $W_{1} \in \mathcal{W}, X_{1} \in \mathcal{X}$, and $Y_{1} \in \mathcal{Y}$ for finite sets $\mathcal{W}, \mathcal{X}$ and $\mathcal{Y}$. The joint distribution of $\left(W_{1}, X_{1}, Y_{1}\right)$ will be arbitrary.

Polarizing such a process may be understood in several ways. One may for instance ask whether a block $\left(W_{1}^{N}, X_{1}^{N}\right)$ can be transformed such that the result $\left(U_{1}^{N}, V_{1}^{N}\right) \in \mathcal{W}^{N} \times \mathcal{X}^{N}$ is polarized in the sense that

$$
H\left(U_{i} V_{i} \mid Y_{1}^{N} U_{1}^{i-1} V_{1}^{i-1}\right) \approx 0 \text { or } \approx 1 \text { for almost all } i \text { 's, }
$$

where the entropy is computed with base- $|\mathcal{W} \times \mathcal{X}|$ logarithms. If no constraints are imposed on this transformation, then it is indeed easy to attain polarization: In light of the results in Section 4, this can be done simply by viewing $\left(W_{1}, X_{1}\right)$ as a single $\mathcal{W} \times \mathcal{X}$-valued random variable, 
and using a polarizing transform for the alphabet $\mathcal{W} \times \mathcal{X}$. Naturally, then, such a definition of joint polarization is not very interesting.

In order to obtain a more useful definition, let us first place the underlying process $\left(W_{1}, X_{1}, Y_{1}\right),\left(W_{2}, X_{2}, Y_{2}\right), \ldots$ in an operational context. As in single source/channel polarization, two simple interpretations are possible:

Separate encoding of correlated sources. In this setting, $W_{1}^{N}$ and $X_{1}^{N}$ can be viewed as the outputs of two correlated i.i.d. sources, which are observed by separate source encoders. The sequence $Y_{1}^{N}$ can be thought of as side information about the source outputs, available to the decoder. The output sequences are encoded separately by their respective encoders, and are subsequently estimated by the decoder. It was shown by Slepian and Wolf [43] that the set of all achievable rate pairs $\left(R_{W}, R_{X}\right)$ in this setup is characterized by the bounds

$$
\begin{aligned}
R_{W} & \geq H\left(W_{1} \mid Y_{1} X_{1}\right) \\
R_{X} & \geq H\left(X_{1} \mid Y_{1} W_{1}\right) \\
R_{W}+R_{X} & \geq H\left(W_{1} X_{1} \mid Y_{1}\right) .
\end{aligned}
$$

Corner points of this region can be achieved by employing a singlesource polar code at each encoder. To see this, consider the corner point $R_{W}=H\left(W_{1} \mid Y_{1}\right), R_{X}=H\left(X_{1} \mid Y_{1} W_{1}\right)$, and the following scheme:

Encoding. The encoders for $W$ and $X$ each choose a polarizing transform for alphabet sizes $|\mathcal{W}|$ and $|\mathcal{X}|$ respectively and compute the sets

$$
\mathcal{A}_{W}=\left\{i: Z\left(U_{i} \mid Y_{1}^{N} U_{1}^{i-1}\right) \approx 0\right\}
$$

and

$$
\mathcal{A}_{X}=\left\{i: Z\left(V_{i} \mid Y_{1}^{N} W_{1}^{N} V_{1}^{i-1}\right) \approx 0\right\} .
$$

Here $U_{1}^{N}$ (respectively, $V_{1}^{N}$ ) is the result of the polarizing transform for $W$ (respectively, $X$ ). Upon observing their corresponding source outputs $W_{1}^{N}$ and $X_{1}^{N}$, both encoders apply their transforms to obtain $U_{1}^{N}$ and $V_{1}^{N}$, and send $U_{\mathcal{A}_{W}^{c}}$, and $V_{\mathcal{A}_{X}^{c}}$ to the decoder.

Decoding. The decoder first estimates $W_{1}^{N}$ from $U_{\mathcal{A}_{W}^{c}}$ and $Y_{1}^{N}$ using the successive cancellation (SC) decoder for the sequence 
$\left(W_{1}, Y_{1}\right),\left(W_{2}, Y_{2}\right), \ldots$ (That is, it ignores its knowledge of $\left.V_{\mathcal{A}_{X}^{c}} \cdot\right)$ It then assumes that its estimate $\hat{W}_{1}^{N}$ is correct and therefore that $\hat{W}_{1}^{N}$ is identically distributed as $W_{1}^{N}$, and uses the SC decoder for the sequence $\left(X_{1},\left(Y_{1} W_{1}\right)\right),\left(X_{2},\left(Y_{2} W_{2}\right)\right), \ldots$ to estimate $X_{1}^{N}$ from $V_{\mathcal{A}_{X}^{c}}$ and $\left(Y_{1}^{N} \hat{W}_{1}^{N}\right)$.

Rate. It follows from single-source polarization theorems that $\left|\mathcal{A}_{W}^{c}\right| \approx$ $N H\left(W_{1} \mid Y_{1}\right)$ and $\left|\mathcal{A}_{X}^{c}\right| \approx N H\left(X_{1} \mid Y_{1} W_{1}\right)$, i.e., that the above scheme operates approximately at a corner point of the achievable region.

Error probability. A decoding error occurs if at least one of the two constituent SC decoders errs. The probability of this event can be upper bounded by the sum of the error probabilities of each decoder. (The proof of this fact is identical to that of Proposition 2.1.) It follows from previous results that each of these average block error probabilities, and thus also their sum, is approximately $2^{-\sqrt{N}}$.

Multiple-access channel. Recall that the capacity region of a multiple-access channel is the convex hull of

$$
\bigcup_{W, X} \mathcal{R}_{W, X}
$$

where

$$
\begin{aligned}
\mathcal{R}_{W, X}=\left\{\left(R_{1}, R_{2}\right):\right. & R_{W} \leq I(W ; Y X) \\
R_{X} & \leq I(X ; Y W) \\
R_{W}+R_{X} & \leq I(W X ; Y)\} .
\end{aligned}
$$

Here $W$ and $X$ are independently distributed inputs to the channel, and $Y$ is the output. The sequence $\left(W_{1}, X_{1}, Y_{1}\right),\left(W_{2}, X_{2}, Y_{2}\right), \ldots$ naturally fits in such a setting. This is best seen by considering the case in which $W_{1}$ and $X_{1}$ are uniformly and independently distributed inputs to the channel, and $Y_{1}$ is the output. The region corresponding to this case is described by the rate bounds

$$
\begin{aligned}
R_{W} & \leq 1-H\left(W_{1} \mid Y_{1} X_{1}\right) \\
R_{X} & \leq 1-H\left(X_{1} \mid Y_{1} W_{1}\right) \\
R_{W}+R_{X} & \leq 2-H\left(W_{1} X_{1} \mid Y_{1}\right) .
\end{aligned}
$$


Corner points of this region can be achieved by the following coding scheme, which is similar to the one for the source coding case:

Code construction. The encoders for $W$ and $X$ each choose a polarizing transform $G_{W}$ and $G_{X}$ for alphabet sizes $|\mathcal{W}|$ and $|\mathcal{X}|$ respectively, and compute the sets

$$
\mathcal{A}_{W}=\left\{i: Z\left(U_{i} \mid Y_{1}^{N} U_{1}^{i-1}\right) \approx 0\right\}
$$

and

$$
\mathcal{A}_{X}=\left\{i: Z\left(V_{i} \mid Y_{1}^{N} W_{1}^{N} V_{1}^{i-1}\right) \approx 0\right\} .
$$

where $U_{1}^{N}=G_{W}\left(W_{1}^{N}\right)$ and $V_{1}^{N}=G_{X}\left(X_{1}^{N}\right)$ are the respective outputs of these transforms. The senders choose $U_{i}, i \in \mathcal{A}_{W}^{c}$ and $V_{i}, i \in \mathcal{A}_{X}^{c}$ independently and uniformly at random and reveal their values to the receiver.

Encoding. Given uniformly distributed messages $M_{W} \in \mathcal{W}^{\left|\mathcal{A}_{W}\right|}$ and $M_{X} \in \mathcal{X}^{\left|\mathcal{A}_{X}\right|}$, the receivers respectively set $U_{\mathcal{A}_{W}}=M_{W}$ and $V_{\mathcal{A}_{X}}=M_{X}$ and transmit $G_{W}^{-1}\left(U_{1}^{N}\right)$ and $G_{X}^{-1}\left(V_{1}^{N}\right)$ over the channel.

Decoding. The decoder first decodes $U_{\mathcal{A}_{W}}$ from $U_{\mathcal{A}_{W}^{c}}$ and $Y_{1}^{N}$ using the SC decoder for the sequence $\left(W_{1}, Y_{1}\right),\left(W_{2}, Y_{2}\right), \ldots$ and produces $\hat{M}_{W}=G_{W}\left(\hat{W}_{1}^{N}\right)$ as its estimate of the message $M_{W}$. It then assumes that this estimate is correct, and uses the $\mathrm{SC}$ decoder for the sequence $\left(X_{1},\left(Y_{1} W_{1}\right)\right),\left(X_{2},\left(Y_{2} W_{2}\right)\right), \ldots$ to decode $V_{\mathcal{A}_{X}}$ from $V_{\mathcal{A}_{X}^{c}}$ and $\left(Y_{1}^{N} \hat{W}_{1}^{N}\right)$, and produces $\hat{M}_{X}=G_{X}\left(\hat{X}_{1}^{N}\right)$ as its estimate of $M_{X}$.

Rate. It follows from previous results that $\left|\mathcal{A}_{W}\right| \approx N\left(1-H\left(W_{1} \mid Y_{1}\right)\right)$ and $\left|\mathcal{A}_{X}\right| \approx N\left(1-H\left(X_{1} \mid Y_{1} W_{1}\right)\right)$, i.e., that the above scheme operates near a corner point of the region given in (6.2).

Error probability. The block error probability is as in the source coding case, i.e., $\approx 2^{-\sqrt{N}}$ averaged over all message pairs and all pairs of frozen vectors $U_{i}, i \in \mathcal{A}_{X}^{c}$ and $V_{i}, i \in \mathcal{A}_{X}^{c}$. It thus follows that there exists at least one frozen vector pair for which the average block error probability is $\approx 2^{-\sqrt{N}}$.

Both of the coding schemes above are obtained by reducing the corresponding multi-user problem into two single-user problems, for 
which devising polar coding schemes is easy. Arbitrary points in the achievable rate region in each problem can be achieved via the 'rate splitting' technique of [17]. (In the multiple-access problem, one can also use the technique discussed in Section 4.3 to achieve rate regions with non-uniform inputs.) Clearly, these schemes can be generalized to settings with more than two users. They also yield an alternative polar coding method for single-sources and point-to-point channels when the source/channel-input alphabet size is a composite number. To see this, consider the sequence $\left(X_{1}, Y_{1}\right),\left(X_{2}, Y_{2}\right), \ldots$ with $X_{1} \in \mathcal{X}$ and $|\mathcal{X}|=q_{1} \cdot q_{2} \ldots \cdot q_{k}$. To polarize $X_{1}, X_{2}, \ldots$, one may — instead of applying a polarizing transform for the alphabet $\mathcal{X}$ directly — view $X_{1}$ as a collection of random variables $\left(X^{(1)}, \ldots, X^{(k)}\right)$ taking values in $\mathcal{X}^{(1)} \times \ldots \times \mathcal{X}^{(k)}$, with $\left|\mathcal{X}^{(i)}\right|=q_{i}$. This decomposition can be made in an arbitrary manner. Considering the expansion

$$
\begin{aligned}
H\left(X_{1} \mid Y_{1}\right) & =H\left(X_{1}^{(1)}, \ldots, X_{1}^{(k)} \mid Y_{1}\right) \\
& =H\left(X_{1}^{(1)} \mid Y_{1}\right)+\cdots+H\left(X_{1}^{(k)} \mid Y_{1}, X_{1}^{(1)}, \ldots, X_{1}^{(k-1)}\right),
\end{aligned}
$$

one easily sees that long blocks of each component $X^{(i)}$ can be polarized separately as above, and can then be decoded in the order $X^{(1)}$, $X^{(2)}, \ldots, X^{(k)}$, using the appropriate SC decoder in each step. Such a scheme also achieves optimal rates in both channel and source coding, with error probabilities comparable to those of direct polarization schemes.

Our aim here is not just to find polar coding schemes for multi-user settings. Instead, we would also like to know whether one can polarize multiple processes jointly in the sense that (a) polarization is achieved by applying a separate transform to the underlying sequences, and that (b) the resulting random variables $\left(\left(U_{i}, V_{i}\right)\right.$ above $)$ are extremal conditioned on their past $\left(U_{1}^{i-1}, V_{1}^{i-1}\right)$, in the sense that they consist only of deterministic and/or uniformly random parts. Observe that our first definition of joint polarization in (6.1) meets requirement (b) but not (a), since a polarizing transform for a single sequence may not necessarily be decomposed into two separate transforms on the constituent sequences. On the other hand, the second polarization method we discussed does meet (a), as it achieves polarization through separately 
applying a transform to each sequence. However, it is not clear at this point that it meets requirement (b), since the joint distributions $p_{U_{i} V_{i} \mid Y_{1}^{N} U_{1}^{i-1} V_{1}^{i-1}}$ one obtains by this method may not be extremal. (We will see that they indeed are.)

This aim can be motivated analogously to single source/channel polarization: In the single-user case, an extremal channel is one whose input is either determined by or independent of its output. In a multiuser setting, a channel may be called extremal if this property holds for all of its inputs: some are determined by the output, others are independent of it. In the two-user case, this is equivalent to saying that an extremal channel (or equivalently, an extremal joint source) is one for which the entropies $H\left(W_{1} \mid Y_{1} X_{1}\right)$ and $H\left(X_{1} \mid Y_{1} W_{1}\right)$ are $\{0,1\}$-valued, and $H\left(W_{1} X_{1} \mid Y_{1}\right)$ is $\{0,1,2\}$-valued. It can easily be seen that there are five possible extremal channels/sources with these properties, the rate regions (6.2) associated with such channels are depicted in Figure 6.1. It is also easily seen that reliable communication over extremal channels is
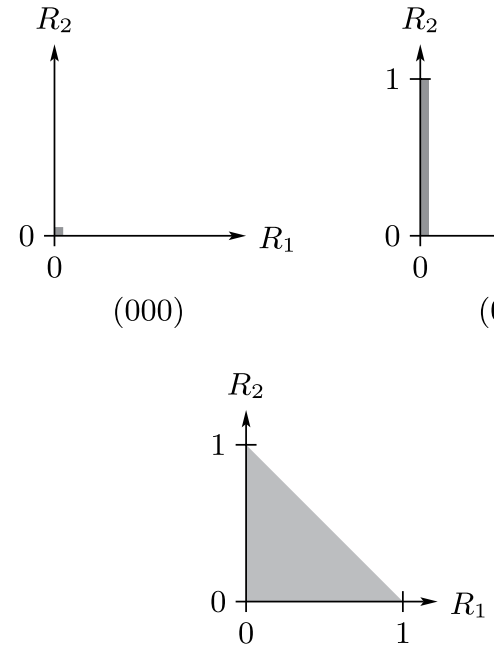

(001)

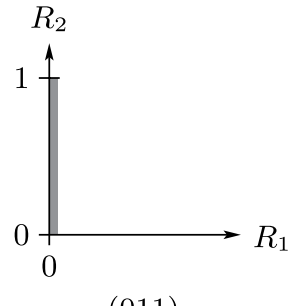

(011)

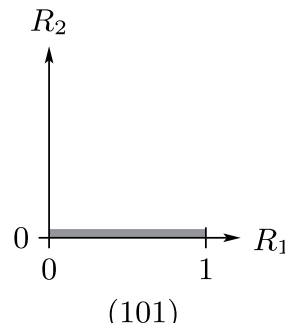

$(101)$

Fig. 6.1 Rate regions of the extremal multiple-access channels (achievable source coding rate regions for extremal sources are analogous to these). (000) is a channel whose inputs are independent from its output, (011) and (101) are channels in which one input is determined by the output and the other is independent from it, (001) is one in which either of the inputs, but not both, can be determined from the output, and (112) is a noiseless multiple-access channel whose inputs are functions of the output. 
trivial, as in the single-user case. Our aim is to polarize several copies of a mediocre multiple-access channel (respectively, joint source) to a set of extremal ones, thereby simplifying the transmission (respectively, compression) task.

\subsection{Joint Polarization}

Consider an i.i.d. process $\left(W_{1}, X_{1}, Y_{1}\right),\left(W_{2}, X_{2}, Y_{2}\right), \ldots$ as above. For notational convenience, we will assume in this section that $\mathcal{W}=\mathcal{X}$ and later discuss how the results here apply to processes with different alphabet sizes. We will be interested in determining how the entropies

$$
\begin{aligned}
H[1] & :=H\left(W_{1} \mid Y_{1} X_{1}\right), \\
H[2] & :=H\left(X_{1} \mid Y_{1} W_{1}\right), \\
H[12] & :=H\left(W_{1} X_{1} \mid Y_{1}\right),
\end{aligned}
$$

which define the achievable rate regions evolve in the course of a joint polarization process. For this purpose, we first choose a polarizing mapping, which we will denote by the generic symbol ' + ', and apply it separately to $\left(W_{1}, W_{2}\right)$ and $\left(X_{1}, X_{2}\right)$ to obtain

$$
\begin{array}{ll}
U_{1}=W_{1}+W_{2}, & V_{1}=X_{1}+X_{2}, \\
U_{2}=W_{2}, & V_{2}=X_{2} .
\end{array}
$$

We also set the following shorthand notation for the resulting entropy terms of interest

$$
\begin{array}{rlrl}
H^{b}[1] & :=H\left(U_{1} \mid Y_{1}^{2} V_{1}\right), & H^{g}[1]:=H\left(U_{2} \mid Y_{1}^{2} U_{1} V_{1} V_{2}\right), \\
H^{b}[2]:=H\left(V_{1} \mid Y_{1}^{2} U_{1}\right), & H^{g}[2]:=H\left(V_{2} \mid Y_{1}^{2} U_{1} V_{1} U_{2}\right), \\
H^{b}[12]:=H\left(U_{1} V_{1} \mid Y_{1}^{2}\right), \quad H^{g}[12]:=H\left(U_{2} V_{2} \mid Y_{1}^{2} U_{1} V_{1}\right) .
\end{array}
$$

If one applies this transform to both sequences recursively in the usual manner, one obtains after $n$ recursions $U_{1}^{N}=G_{N}\left(W_{1}^{N}\right)$ and $V_{1}^{N}=G_{N}\left(X_{1}^{N}\right)$, where again $N=2^{n}$ and $G_{N}$ represents $n$ recursions of the polarizing transform. Our aim is to show that the resulting random variable triples $\left(U_{i}, V_{i},\left(Y_{1}^{N} U_{1}^{i-1} V_{1}^{i-1}\right)\right)$ are polarized in the sense 
that for all $\epsilon>0$, we have

$$
\begin{aligned}
H^{(i)}[1] & :=H\left(U_{i} \mid Y_{1}^{N} U_{1}^{i-1} V_{1}^{i-1} V_{i}\right) \notin(\epsilon, 1-\epsilon), \\
H^{(i)}[2]: & =H\left(V_{i} \mid Y_{1}^{N} U_{1}^{i-1} V_{1}^{i-1} U_{i}\right) \notin(\epsilon, 1-\epsilon), \\
H^{(i)}[12]: & =H\left(U_{i} V_{i} \mid Y_{1}^{N} U_{1}^{i-1} V_{1}^{i-1}\right) \notin(\epsilon, 1-\epsilon) \cup(1+\epsilon, 2-\epsilon),
\end{aligned}
$$

for almost all $i \in\{1, \ldots, N\}$, provided that $N$ is sufficiently large. This is equivalent to saying that the entropy triples $\left(H^{(i)}[1], H^{(i)}[2], H^{(i)}[12]\right)$ for almost all $i$ 's is close to one of the five extremal values

$$
(0,0,0), \quad(0,1,1), \quad(1,0,1), \quad(0,0,1), \quad(1,1,2) .
$$

As in the previous sections, the main ingredient of the proof of this polarization statement is a result on the single-step evolution of entropies $H[1], H[2]$, and $H[12]$ :

Lemma 6.1. For every $\epsilon>0$, there exists $\delta>0$ such that

$$
H^{b}[12]-H[12] \leq \delta
$$

implies

(i) $H^{b}[1]-H[1] \leq \delta$ and $H^{b}[2]-H[2] \leq \delta$,

(ii) $H[1], H[2] \notin(\epsilon, 1-\epsilon)$,

(iii) $H[12] \notin(2 \epsilon, 1-\epsilon) \cup(1+\epsilon, 2-2 \epsilon)$.

Proof. We have

$$
\begin{aligned}
\delta \geq & H^{b}[12]-H[12] \\
= & H\left(W_{1}+W_{2}, X_{1}+X_{2} \mid Y_{1}^{2}\right)-H\left(W_{1} X_{1} \mid Y_{1}\right) \\
= & H\left(W_{1}+W_{2} \mid Y_{1}^{2}\right)-H\left(W_{1} \mid Y_{1}\right) \\
& +H\left(X_{1}+X_{2} \mid Y_{1}^{2}, W_{1}+W_{2}\right)-H\left(X_{1} \mid Y_{1} W_{1}\right)
\end{aligned}
$$

Note that both entropy differences in (6.4) are non-negative, and thus are at most $\delta$, implying $H^{b}[2]-H[2] \leq \delta$. Swapping the $W$ 's and the 
$X$ 's in the above relations also yields $H^{b}[1]-H[1] \leq \delta$, proving (i). One can continue (6.4) as

$$
\begin{aligned}
\delta \geq & H\left(W_{1}+W_{2} \mid Y_{1}^{2}\right)-H\left(W_{1} \mid Y_{1}\right) \\
& +H\left(X_{1}+X_{2} \mid Y_{1}^{2} W_{1}^{2}\right)-H\left(X_{1} \mid Y_{1} W_{1}\right) .
\end{aligned}
$$

For sufficiently small $\delta$, it follows from (6.5) and Theorem 4.12 that $H\left(W_{1} \mid Y_{1}\right) \notin(\epsilon, 1-\epsilon)$, and $H\left(X_{1} \mid Y_{1} W_{1}\right)=H[2] \notin(\epsilon, 1-\epsilon)$. Further, since

$$
H\left(W_{1} X_{1} \mid Y_{1}\right)=H\left(W_{1} \mid Y_{1}\right)+H\left(X_{1} \mid Y_{1} W_{1}\right)
$$

it follows that $H\left(W_{1} X_{1} \mid Y_{1}\right)=H[12] \notin(2 \epsilon, 1-\epsilon) \cup(1+\epsilon, 2-2 \epsilon)$, yielding (iii). By swapping the $X$ 's with the $W$ 's in the above chain of inequalities one also obtains $H\left(X_{1} \mid Y_{1}\right) \notin(\epsilon, 1-\epsilon)$ and $H\left(W_{1} \mid\right.$ $\left.Y_{1} X_{1}\right)=H[1] \notin(\epsilon, 1-\epsilon)$, completing the proof.

This lemma suffices to show the main polarization result of this section.

Theorem 6.2. Let $M:=\{(0,0,0),(0,1,1),(1,0,1),(0,0,1),(1,1,2)\}$, and

$$
d(a, M):=\max _{b \in M}\|a-b\|, \quad a \in \mathbb{R}^{3} .
$$

For all $\epsilon>0$, we have

$$
\lim _{n \rightarrow \infty} \frac{1}{N}\left|\left\{i: d\left(\left(H^{(i)}[1], H^{(i)}[2], H^{(i)}[12]\right), M\right) \geq \epsilon\right\}\right|=0 .
$$

Proof. The proof is similar to those of previous polarization theorems: Let $B_{1}, B_{2}, \ldots$ be an i.i.d. process with $\operatorname{Pr}\left[B_{1}=b\right]=\operatorname{Pr}\left[B_{1}=g\right]=1 / 2$. Define a process $\left(H_{0}[1], H_{0}[2], H_{0}[12]\right),\left(H_{1}[1], H_{1}[2], H_{1}[12]\right), \ldots$ with

$$
\begin{aligned}
& H_{0}[k]=H[k], \\
& H_{n}[k]=H_{n-1}^{B_{n}}[k], \quad n=1,2, \ldots,
\end{aligned}
$$

for $k=1,2,12$. Observe that

$$
\begin{aligned}
H^{b}[12]+H^{g}[12] & =H\left(U_{1} V_{1} \mid Y_{1}^{2}\right)+H\left(U_{2} V_{2} \mid Y_{1}^{2} U_{1} V_{1}\right) \\
& =H\left(W_{1}^{2} X_{1}^{2} \mid Y_{1}^{2}\right) \\
& =2 H[12]
\end{aligned}
$$


therefore the process $H_{0}[12], H_{1}[12], \ldots$ is a bounded martingale and converges almost surely to a [0,2]-valued random variable $H_{\infty}[12]$. It then follows from (i) in Lemma 6.1 that processes $H_{0}[1], H_{1}[1], \ldots$ and $H_{0}[2], H_{1}[2], \ldots$ also converge almost surely to $[0,1]$-valued random variables $H_{\infty}[1]$ and $H_{\infty}[2]$, respectively. It further follows from (ii) in Lemma 6.1 that $H_{\infty}[1]$ and $H_{\infty}[2]$ are $\{0,1\}$-valued, and from (iii) that $H_{\infty}[12]$ is $\{0,1,2\}$-valued, i.e., that the process $\left(H_{0}[1], H_{0}[2], H_{0}[12]\right),\left(H_{1}[1], H_{1}[2], H_{1}[12]\right), \ldots$ converges almost surely to a random vector taking values in the set $M$. The claim then follows from the equivalence between the probability distribution of $\left(H_{n}[1], H_{n}[2], H_{n}[12]\right)$ and the distribution of $\left(H^{(i)}[1], H^{(i)}[2], H^{(i)}[12]\right)$, $i=1, \ldots, N$.

\subsubsection{Rate Region}

We have seen that separately applying a polarizing transformation to two i.i.d. processes polarizes them jointly, i.e., the resulting joint distributions approach one of five extremal distributions as the construction size grows. We now consider the rate region obtained by this procedure. We will discuss the multiple-access channel interpretation of the result.

Let $\mathcal{R}$ denote the rate region defined by the bounds in (6.2). Also let $\mathcal{R}^{b}$ and $\mathcal{R}^{g}$ denote the rate regions obtained after the first polarization step, i.e., those with entropies $(H[1], H[2], H[12])$ in (6.2) replaced respectively by $\left(H^{b}[1], H^{b}[2], H^{b}[12]\right)$ and $\left(H^{g}[1], H^{g}[2], H^{g}[12]\right)$. One can similarly define the regions $\mathcal{R}^{\mathbf{s}}, \mathbf{s} \in\{b, g\}^{n}$ obtained after $n$ polarization steps. Note that

$$
\begin{aligned}
2 H[1] & =H\left(W_{1}^{2} \mid Y_{1}^{2} X_{1}^{2}\right) \\
& =H\left(U_{1}^{2} \mid Y_{1}^{2} V_{1}^{2}\right) \\
& \leq H\left(U_{1} \mid Y_{1}^{2} V_{1}\right)+H\left(U_{2} \mid Y_{1}^{2} U_{1} V_{1} V_{2}\right) \\
& =H^{b}[1]+H^{g}[1] .
\end{aligned}
$$

It similarly follows that

$$
\begin{gathered}
2 H[2] \leq H^{b}[2]+H^{g}[2], \\
2 H[12]=H^{b}[12]+H^{g}[12],
\end{gathered}
$$




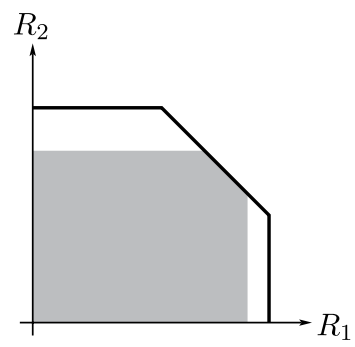

Fig. 6.2 The average of the rate regions after $n$ polarization steps (the shaded region) is a subset of the original region, but contains points on the dominant face of the latter.

and therefore the set

$$
\frac{1}{2} \mathcal{R}^{b}+\frac{1}{2} \mathcal{R}^{g}=\left\{\frac{1}{2} a+\frac{1}{2} b: a \in \mathcal{R}^{b}, b \in \mathcal{R}^{g}\right\}
$$

is a subset of $\mathcal{R}$. It is easy to find examples where this inclusion is strict. Nevertheless, due to equality in (6.6) and the polymatroidal nature of $\mathcal{R}, \frac{1}{2} \mathcal{R}^{b}+\frac{1}{2} \mathcal{R}^{g}$ and $\mathcal{R}$ share points on their dominant faces (see Figure 6.2). Polarizing the resulting regions $\mathcal{R}^{b}$ and $\mathcal{R}^{g}$ further will similarly lead to a loss of overall rate region, i.e., for all $n$

$$
\frac{1}{N} \sum_{\mathbf{s} \in\{b, g\}^{n}} \mathcal{R}^{\mathbf{s}} \subset \mathcal{R}
$$

although the regions on either side of the last relation will share at least one point on their dominant faces. Note that the situation here is in contrast with point-to-point channel polarization, where no rate penalty is incurred by the construction.

\subsubsection{Processes with Different Alphabet Sizes}

We have so far assumed that the processes we polarize jointly have identical alphabet sizes. However, this restriction is only for notational convenience, and is not necessary for polarization to take place. It can indeed be seen easily that the proofs given above are equally valid when the alphabet sizes of the processes differ, and the resulting random variables are still either uniformly random or deterministic. If one computes entropies with base- $|\mathcal{W}||\mathcal{X}|$ logarithms, then the extremal 
values for $(H[1], H[2], H[12])$ become

$(0,0,0), \quad(0, \log |\mathcal{X}|, \log |\mathcal{X}|) \quad(\log |\mathcal{W}|, 0, \log |\mathcal{W}|), \quad(\log |\mathcal{W}|, \log |\mathcal{X}|, 1)$, corresponding respectively to the previous cases (000), (011), (101), (112). The case (001) is precluded from this setting. To see the reason for this, suppose that random variables $(W, X, Y)$ with $|\mathcal{W}|<|\mathcal{X}|$ satisfy the conditions of the case (001): $X$ is uniformly distributed conditioned on $Y$, but is a function of $(W, Y)$, i.e., $H(X \mid Y)=\log |\mathcal{X}|$ and $H(X \mid$ $Y W)=0$. This would imply $I(W ; X \mid Y)=\log |\mathcal{X}|$, an impossibility since $I(W ; X \mid Y) \leq \log |\mathcal{W}|$. Consequently, the rate region obtained by polarization is rectangular (i.e., it has a single point on the dominant face of the original region) when the alphabet sizes differ.

\subsection{Rate of Polarization}

Our purpose in this section is to give operational meaning to the rate region obtained after polarization. We will do so by describing a channel coding scheme that achieves the corresponding rate region - the source coding counterpart is similar. We will restrict our attention to processes with prime alphabet sizes, and will assume that the polarizing mapping ' + ' for each alphabet is the corresponding modulo-addition operation.

Suppose $W_{1}, W_{2}, \ldots$ and $X_{1}, X_{2}, \ldots$ are i.i.d., uniformly distributed inputs to a multiple-access channel, and $Y_{1}, Y_{2}, \ldots$ is the output. Let $G_{X}$ and $G_{W}$ be two polarizing transforms as above, and $U_{1}^{N}=G_{W}\left(W_{1}^{N}\right)$, $V_{1}^{N}=G_{X}\left(X_{1}^{N}\right)$ their outputs. Fix $\epsilon>0$, and define the set

$$
\mathcal{P}_{\epsilon}(a, b, c):=\left\{i:\left\|\left(H^{(i)}[1], H^{(i)}[2], H^{(i)}[12]\right)-(a, b, c)\right\|<\epsilon\right\}
$$

for $(a, b, c) \in \mathbb{R}^{3}$. Let $\mathcal{A}_{W}, \mathcal{A}_{X} \subset\{1, \ldots, N\}$ denote sets of indices over which the users transmit their data, and choose these sets as follows:

(i.a) If $i \in \mathcal{P}_{\epsilon}(0,0,0)$, then set $i \in \mathcal{A}_{W}, i \in \mathcal{A}_{X}$,

(i.b) else if $i \in \mathcal{P}_{\epsilon}(0,1,1)$, then set $i \in \mathcal{A}_{W}, i \notin \mathcal{A}_{X}$,

(i.c) else if $i \in \mathcal{P}_{\epsilon}(1,0,1)$, then set $i \notin \mathcal{A}_{W}, i \in \mathcal{A}_{X}$,

(i.d) else if $i \in \mathcal{P}_{\epsilon}(0,0,1)$, then set either $i \in \mathcal{A}_{W}, i \notin \mathcal{A}_{X}$ or $i \notin$ $\mathcal{A}_{W}, i \in \mathcal{A}_{X}$,

(ii) else, set $i \notin \mathcal{A}_{W}, i \notin \mathcal{A}_{X}$. 
The senders set $U_{i}, i \in \mathcal{A}_{W}$ and $V_{i}, i \in \mathcal{A}_{X}$ to be the uniformly distributed data symbols. Symbols in $\mathcal{A}_{W}^{c}$ and $\mathcal{A}_{X}^{c}$ are frozen, i.e., they are chosen uniformly at random and revealed to the receiver. It follows from previous results that for all $\epsilon>0$ there exists $N_{0}$ such that $\left|\mathcal{A}_{W}\right|+\left|\mathcal{A}_{X}\right|>N\left(2-H\left(W_{1} X_{1} \mid Y_{1}\right)\right)$ for all $N \geq N_{0}$, i.e., that the operating point of this scheme is close to the dominant face of the original region. The whole dominant face of the region obtained by polarization can be spanned by varying the sizes of the data sets $\mathcal{A}_{W}$ and $\mathcal{A}_{X}$ through (i.d).

Decoding is performed successively as in the single-user case, in the order $\left(U_{1}, V_{1}\right),\left(U_{2}, V_{2}\right), \ldots\left(U_{N}, V_{N}\right)$ : in decoding $\left(U_{i}, V_{i}\right)$ the receiver first sets the frozen symbol (if there is one), say $U_{i}$, to its known value, and decodes $V_{i}$ using the optimal decision rule for the channel $V_{i} \rightarrow Y_{1}^{N} U_{1}^{i-1} V_{1}^{i-1} U_{i}$. If neither $U_{i}$ nor $V_{i}$ is frozen, then they are decoded in an arbitrary order, also using the optimal decision rules for the corresponding channels. Since these channels have the same recursive structure as in the single-user case, the complexity of the described decoding operation is $O(N \log N)$. The error probability of this scheme can similarly be bounded by those of the resulting channels:

$$
\begin{aligned}
P_{\mathrm{e}} \leq & \sum_{i \in \mathcal{P}_{\epsilon}(0,0,0)}\left[Z\left(U_{i} \mid Y_{1}^{N} U_{1}^{i-1} V_{1}^{i-1}\right)+Z\left(V_{i} \mid Y_{1}^{N} U_{1}^{i-1} V_{1}^{i-1}\right)\right] \\
& +\sum_{i \in \mathcal{P}_{\epsilon}(0,1,1)} Z\left(U_{i} \mid Y_{1}^{N} U_{1}^{i-1} V_{1}^{i-1}\right)+\sum_{i \in \mathcal{P}_{\epsilon}(1,0,1)} Z\left(V_{i} \mid Y_{1}^{N} U_{1}^{i-1} V_{1}^{i-1}\right) \\
& +\sum_{i \in \mathcal{P}_{\epsilon}(0,0,1)} \max \left\{Z\left(U_{i} \mid Y_{1}^{N} U_{1}^{i-1} V_{1}^{i-1} V_{i}\right), Z\left(V_{i} \mid Y_{1}^{N} U_{1}^{i-1} V_{1}^{i-1} U_{i}\right)\right\}
\end{aligned}
$$

Note that the Bhattacharyya parameters on the first two lines of the above sum are larger than those of the corresponding channels, since they each ignore the knowledge of one symbol $\left(U_{i}\right.$ or $\left.V_{i}\right)$ available at the output. We will see that this relaxation greatly simplifies error probability proofs. In particular, we will see that almost all Bhattacharyya parameters in the above sum are 'exponentially small', and therefore one can make the sum vanish by freezing a negligible fraction of the data symbols in both codes: 
$\overline{\text { Lemma 6.3. Define } Z^{\prime}(A \mid B):=Z\left(A \mid Y_{1}^{N} U_{1}^{i-1} V_{1}^{i-1} B\right) \text {. There exists }}$ an $\epsilon>0$ such that for all $\beta<1 / 2$,

$$
\begin{aligned}
& \lim _{n \rightarrow \infty} \frac{1}{N}\left|\left\{i \in \mathcal{P}_{\epsilon}(0,0,0): Z^{\prime}\left(U_{i}\right)+Z^{\prime}\left(V_{i}\right) \geq 2^{-N^{\beta}}\right\}\right|=0, \\
& \lim _{n \rightarrow \infty} \frac{1}{N}\left|\left\{i \in \mathcal{P}_{\epsilon}(0,1,1): Z^{\prime}\left(U_{i}\right) \geq 2^{-N^{\beta}}\right\}\right|=0, \\
& \lim _{n \rightarrow \infty} \frac{1}{N}\left|\left\{i \in \mathcal{P}_{\epsilon}(1,0,1): Z^{\prime}\left(V_{i}\right) \geq 2^{-N^{\beta}}\right\}\right|=0, \\
& \lim _{n \rightarrow \infty} \frac{1}{N}\left|\left\{i \in \mathcal{P}_{\epsilon}(0,0,1): \max \left\{Z^{\prime}\left(U_{i} \mid V_{i}\right), Z^{\prime}\left(V_{i} \mid U_{i}\right)\right\} \geq 2^{-N^{\beta}}\right\}\right|=0 .
\end{aligned}
$$

Proof. It is easy to see that

(i) $i \in \mathcal{P}_{\epsilon}(0,0,0)$ implies $Z^{\prime}\left(U_{i}\right), Z^{\prime}\left(V_{i}\right) \leq \delta(\epsilon)$,

(ii) $i \in \mathcal{P}_{\epsilon}(0,1,1)$ implies $Z^{\prime}\left(U_{i}\right) \leq \delta(\epsilon)$,

(iii) $i \in \mathcal{P}_{\epsilon}(1,0,1)$ implies $Z^{\prime}\left(V_{i}\right) \leq \delta(\epsilon)$,

(iv) $i \in \mathcal{P}_{\epsilon}(0,0,1)$ implies $Z^{\prime}\left(U_{i} \mid V_{i}\right), Z^{\prime}\left(V_{i} \mid U_{i}\right) \leq \delta(\epsilon)$,

where $\delta(\epsilon) \rightarrow 0$ as $\epsilon \rightarrow 0$. Therefore, the proof will be complete once we show that whenever the above Bhattacharyya parameters are close to 0 , they are exponentially small in the square root of the blocklength. For this purpose, we will define stochastic processes that mirror the behavior of the Bhattacharyya parameters of interest, in the now-customary manner: We first define the Bhattacharyya parameters

$$
\begin{aligned}
& Z^{b}\left(W_{1} \mid Y_{1}\right):=Z\left(W_{1}+W_{2} \mid Y_{1}^{2}\right), \\
& Z^{g}\left(W_{1} \mid Y_{1}\right):=Z\left(W_{2} \mid Y_{1}^{2}, W_{1}+W_{2}, X_{1}+X_{2}\right),
\end{aligned}
$$

obtained from $Z\left(W_{1} \mid Y_{1}\right)$ after the first polarization step. Also define an i.i.d. process $B_{1}, B_{2}, \ldots$ with $\operatorname{Pr}\left[B_{1}=g\right]=\operatorname{Pr}\left[B_{1}=b\right]=1 / 2$, and the processes

$$
\begin{aligned}
& Z_{0}=Z\left(W_{1} \mid Y_{1}\right), \\
& Z_{n}=Z_{n-1}^{B_{n}}, \quad n=1,2, \ldots
\end{aligned}
$$


It suffices to characterize the one-step evolution of the Bhattacharyya parameters, the rest of the proof being identical to previous ones (e.g., Theorem 4.10): observe that

$$
\begin{aligned}
& Z^{b}\left(W_{1} \mid Y_{1}\right)=Z\left(W_{1} \mid Y_{1}\right)^{-} \\
& Z^{g}\left(W_{1} \mid Y_{1}\right) \leq Z\left(W_{2} \mid Y_{1}^{2}, W_{1}+W_{2}\right)=Z\left(W_{1} \mid Y_{1}\right)^{+},
\end{aligned}
$$

where $Z^{-}$and $Z^{+}$are defined as in the single-user case. Consequently, whenever $Z_{n}$ converges to 0 , it does so at least as fast as in singleuser polarization. That is, whenever $Z^{\prime}\left(U_{i}\right)$ is close to 0 , it is almost surely exponentially small in the square root of the blocklength. By symmetry, a similar statement also holds for $Z^{\prime}\left(V_{i}\right)$. This yields the first three claims.

The last claim is trivial when $|\mathcal{W}| \neq|\mathcal{X}|$, since we then have

$$
\lim _{n \rightarrow \infty} \frac{1}{N}\left|\mathcal{P}_{\epsilon}(0,0,1)\right|=0 \text {. }
$$

(See Section 6.1.2.) For the case $|\mathcal{W}|=|\mathcal{X}|$, we will prove that the claimed rate of convergence holds for the Bhattacharyya parameter $Z^{\prime}\left(U_{i}+\alpha V_{i}\right)$, for some $\alpha \in \mathcal{W} \in \backslash\{0\}$ from which the result will follow since

$$
Z^{\prime}\left(U_{i} \mid V_{i}\right)=Z^{\prime}\left(U_{i}+\alpha V_{i} \mid V_{i}\right) \leq Z^{\prime}\left(U_{i}+\alpha V_{i}\right) .
$$

Consider the one-step evolution of the entropy $H\left(W_{1}+\alpha X_{1} \mid Y_{1}\right)$. We have

$$
\begin{aligned}
H^{b}\left(W_{1}+\alpha X_{1} \mid Y_{1}\right) & :=H\left(\left(W_{1}+\alpha X_{1}\right)+\left(X_{2}+\alpha W_{2}\right) \mid Y_{1}^{2}\right), \\
& =H\left(W_{1}+\alpha X_{1} \mid Y_{1}\right)^{-},
\end{aligned}
$$

and

$$
\begin{aligned}
H^{g}\left(W_{1}+\alpha X_{1} \mid Y_{1}\right) & :=H\left(W_{2}+\alpha X_{2} \mid Y_{1}^{2}, W_{1}+W_{2}, X_{1}+X_{2}\right), \\
& \leq H\left(W_{1}+\alpha X_{1} \mid Y_{1}^{2},\left(W_{1}+W_{2}\right)+\alpha\left(X_{1}+X_{2}\right)\right), \\
& =H\left(W_{1}+\alpha X_{1} \mid Y_{1}^{2},\left(W_{1}+\alpha X_{1}\right)+\left(W_{2}+\alpha X_{2}\right)\right), \\
& =H\left(W_{1}+\alpha X_{1} \mid Y_{1}\right)^{+} .
\end{aligned}
$$

If one defines an entropy process $H_{0}, H_{1}, \ldots$ that tracks the evolution of $H\left(W_{1}+\alpha X_{1} \mid Y_{1}\right)$ in the course of the polarization procedure, 
then it can be shown using the above relations that $H_{0}, H_{1}, \ldots$ is a supermartingale and converges almost surely to a $\{0,1\}$-valued random variable. Moreover, it is easily seen that the above chain of relations also holds with entropies replaced by the Bhattacharyya parameters, and thus we have

$$
\begin{aligned}
& Z^{b}\left(W_{1}+\alpha X_{1} \mid Y_{1}\right)=Z\left(W_{1}+\alpha X_{1} \mid Y_{1}\right)^{-} \\
& Z^{g}\left(W_{1}+\alpha X_{1} \mid Y_{1}\right) \leq Z\left(W_{1}+\alpha X_{1} \mid Y_{1}\right)^{+} .
\end{aligned}
$$

Defining once again a Bhattacharyya process $Z_{0}, Z_{1}, \ldots$ in the usual manner, it follows that whenever $Z_{n}$ converges to 0 , it does so at least as fast as in the single-user case. It further follows from Lemma 6.5 in Appendix 6.A that for sufficiently large $N$,

$i \in \mathcal{P}_{\epsilon}(0,0,1)$ implies $Z^{\prime}\left(U_{i}+\alpha V_{i}\right) \leq \delta(\epsilon)$ for some $\alpha \in \mathcal{W} \backslash\{0\}$, where $\delta(\epsilon) \rightarrow 0$ as $\epsilon \rightarrow 0$. We therefore have,

$$
\lim _{n \rightarrow \infty} \frac{1}{N}\left\{i \in \mathcal{P}_{\epsilon}(0,0,1): Z^{\prime}\left(U_{i}+\alpha V_{i}\right) \geq 2^{-N^{\beta}}\right\}=0
$$

for sufficiently small $\epsilon>0$ and all $\beta<1 / 2$, completing the proof.

Corollary 6.4. The average block error probability of the coding scheme described above is $o\left(2^{-N^{\beta}}\right)$ for all $\beta<1 / 2$.

\section{A Appendix}

Lemma 6.5. Let $W, X, Y$ be random variables with $W, X \in \mathcal{W}=\mathbb{F}_{q}$. There exists $\delta>0$ such that

(i) $H(W \mid Y)>1-\delta, \quad H(X \mid Y)>1-\delta, \quad H(W \mid Y X)<\delta$, $H(X \mid Y W)<\delta$ and

(ii) $H(W+\alpha X \mid Y) \notin(\delta, 1-\delta)$ for all $\alpha \in \mathcal{W} \backslash\{0\}$, imply

$$
H\left(W+\alpha^{\prime} X \mid Y\right)<\delta
$$

for some $\alpha^{\prime} \in \mathcal{W}$. 
Proof. Let $\pi$ be a permutation on $\mathcal{W}$, and let

$$
p_{\pi}(w, x)=\left\{\begin{array}{ll}
\frac{1}{q}, & \text { if } w=\pi(x) \\
0, & \text { otherwise }
\end{array} .\right.
$$

Note that $H(W)=H(X)=1$ and $H(W \mid X)=H(X \mid W)=0$ whenever the joint distribution of $(W, X)$ is $p_{\pi}$. We claim that for every $\pi$, there exists an $\alpha_{\pi} \in \mathcal{W} \backslash\{0\}$ such that

$$
H\left(W+\alpha_{\pi} X\right)<1-c(q),
$$

where $c(q)>0$ depends only on $q$. To see this, given a permutation $\pi$, let

$$
\alpha_{\pi}:=\pi(0)-\pi(1)
$$

Clearly, $\alpha_{\pi} \neq 0$. It is also easy to check that with these definitions we have

$$
\begin{aligned}
\operatorname{Pr}\left[W+\alpha_{\pi} X\right. & =\pi(0)], \\
& \geq \operatorname{Pr}[(W, X)=(\pi(0), 0)]+\operatorname{Pr}[(W, X)=(\pi(1), 1)], \\
& =\frac{2}{q},
\end{aligned}
$$

which yields the claim. It also follows from the continuity of entropy in the $L_{1}$ metric that

$$
\left\|p_{W X}-p_{\pi}\right\| \leq o(\delta) \quad \text { implies } H\left(W+\alpha_{\pi} X\right) \leq 1-c(q)+o(\delta) .
$$

We claim that the conditions of the lemma imply that with high probability (on $Y$ ) the distance

$$
\left\|p_{W X \mid Y=y}-p_{\pi}\right\| \text { is small for some } \pi \text {. }
$$

Note first that

$$
\begin{aligned}
\delta>1-H(W \mid Y) & =\sum_{y} p(y)[1-H(W \mid Y=y)], \\
& =\sum_{y} p(y) D\left(p_{W \mid Y=y} \| \operatorname{uni}(\mathcal{W})\right), \\
& \geq \sum_{y} p(y) \frac{1}{2}\left\|p_{W \mid Y=y}-\operatorname{uni}(\mathcal{W})\right\|^{2},
\end{aligned}
$$


where the last relation is a consequence of Pinsker's inequality. It then follows that the set

$$
G=\left\{y:\left\|p_{W \mid Y=y}-\operatorname{uni}(\mathcal{W})\right\|<\delta^{1 / 4}\right\}
$$

has probability at least $1-2 \delta^{1 / 4}$. Further, as

$$
\delta>H(X \mid W Y)=\sum_{y} p_{Y}(y) H(X \mid W, Y=y)
$$

the set $B=\{y: H(X \mid W, Y=y) \leq \sqrt{\delta}\}$ has probability at least $1-$ $\sqrt{\delta}$. Hence, set $S=G \cap B$ has probability at least $1-2 \delta^{1 / 4}-\sqrt{\delta}$. Note that for all $y \in S$ we have for any $w,\left|\frac{1}{q}-p_{W \mid Y=y}(w)\right|<o(\delta)$, and $p_{X \mid W Y}(x \mid w, y) \notin(o(\delta), 1-o(\delta))$, and thus

$$
\min _{\pi}\left\|p_{W X \mid Y=y}-p_{\pi}\right\|<o(\delta),
$$

yielding the claim in (6.8). In particular, this implies that there exist $\pi^{\prime}$ and $S^{\prime} \subset S$ with $p_{Y}\left(S^{\prime}\right) \geq p_{Y}(S) / q$ ! such that

$$
\left\|p_{W X \mid Y=y}-p_{\pi^{\prime}}\right\|<o(\delta),
$$

for all $y \in S^{\prime}$. Choosing $\alpha^{\prime}=\alpha_{\pi^{\prime}}$ as in (6.7), we obtain

$$
\begin{aligned}
H\left(W+\alpha^{\prime} X \mid Y\right) & \leq p_{Y}\left(S^{\prime}\right)(1-c(q)+o(\delta))+p_{Y}\left(S^{\prime c}\right), \\
& =1-c_{2}+o(\delta),
\end{aligned}
$$

where $c_{2}>0$ depends only on $q$. Since $H\left(W+\alpha^{\prime} X \mid Y\right) \notin(\delta, 1-\delta)$ by assumption, and we see that if $\delta$ is sufficiently small, then $H\left(W+\alpha^{\prime} X \mid\right.$ $Y) \leq \delta$. 


\section{Conclusion and Related Work}

In Section 2, we started by studying a two-by-two combining/splitting mapping, which transforms two uses of a binary-input memoryless channel (respectively, two outputs of a memoryless binary source) into two channels with unequal capacities (respectively, two random variables with unequal entropies). We then used this mapping recursively to amplify the difference between the channels (respectively, sources), and showed that in the limit, this recursion creates only perfect channels and useless channels (respectively, constant random variables and uniformly distributed random variables). We then saw that the convergence of the created channels and sources to the limiting values is fast - almost exponential in the square root of the blocklength. It is worth noting that this result on the rate of convergence and thus on the error probability of polar coding is asymptotic, and one may need to go to impractically large blocklengths to attain the promised exponential decay in the error probabilities. We also saw empirical evidence for the unimpressive performance of successive cancellation decoding at small blocklengths. Fortunately, this evidence also suggested that the error performance can be improved significantly via simple modifications to the decoding algorithm, while keeping the computational complexity 
at practical levels. All of the results in this section are from $[4,5]$, and [9], while the error probability plots for successive cancellation and list decoding are from [44].

In Section 3, we provided further evidence for the practical relevance of polar codes. The encoding and the successive cancellation decoding (time and space) complexities were shown to be $O(N \log N)$. We also saw an $O(N \log N)$ complexity algorithm, described in [45], to construct good polar codes for arbitrary channel and source models. Construction of polar codes was first discussed in [4], and also in [33]. Crucial to the low complexity figures for all algorithms in this section was the recursive nature of encoding, decoding, and the descriptions of polarized channels/sources.

In Section 4, we studied polarization for non-binary processes. Achieving polarization for finite fields with randomized transforms was first discussed in the original work of Arıkan [4]. We showed that discrete memoryless processes with prime alphabet sizes can be polarized by a recursive linear transform similar to the original one for binary processes. We saw that linear transforms fail to polarize all memoryless processes with composite alphabet sizes. These were first proved in [40]; the proof we saw is from [39]. We then demonstrated a family of nonlinear transforms that polarize stationary memoryless processes with arbitrary discrete alphabets. The crucial property of all basic polarizing transforms is their ability to create a high-entropy and a low-entropy random variable out of two moderate-entropy random variables, irrespective of the distribution of the latter. We also derived 'exponential' error probability bounds for channel codes (respectively, source codes) based on the proposed transforms, establishing their capacity-achieving (respectively, entropy-achieving) properties. Since the results there hold for codes on all discrete alphabets, one can approach the capacity of any memoryless channel with continuous inputs by approximating its capacity-achieving input distribution through the method discussed in Section 4.3.

It is worth mentioning that several methods have been proposed to construct polar codes for non-binary alphabets. We discussed one such method in Section 6, which was based on factorizing the alphabet into smaller alphabets and polarizing these successively. Techniques that 
achieve similar (but not identical) multilevel polarization effects were proposed in [2] and [35].

The results in Section 5 were obtained in [26] for the binary case. Generalizations to the non-binary case were given in [34] and [39]. Here, we first showed that processes with prime alphabet sizes can be polarized by any linear transform whose matrix representation is not upper-triangular. This also implies that given any invertible and non-trivial transform, one can find a decoding order (i.e., a permutation of the columns of the transform) under which the resulting random variables are polarized. We observed that the exponential error probability behavior of recursive polar codes is closely related to the distance properties of a single recursion. We derived a simple formula that characterizes this behavior. Although we only provided upper bounds on the error probability in terms of this formula, one can in fact show that the minimum distance behavior of polar codes is given by the same formula, and conclude that successive cancellation decoding of polar codes achieves optimal performance in the exponential sense. We also saw that the error probability improvements afforded by general constructions over Arıkan's original construction is significant especially for larger alphabet sizes. One should note, however, that the results on the error probability are asymptotic, as are the results in Section 2, and are not very informative about the performance of short polar codes. Two problems of interest in this direction are to determine whether generalized transforms yield stronger codes at practically relevant lengths, and to determine whether reliability gains can be attained by using non-binary polar codes over binary channels. To that end, one can use a generalized version of the algorithm given in [45] to evaluate the performance of various polar code constructions on various channels, although it is also of interest to develop a theory of polar code design for practically relevant blocklengths.

In Section 6 we considered polarization for multi-user coding settings. We first showed that all optimal rates for multiple-access channels and the distributed source coding problems can be achieved using polar codes at each user. This was first observed in [22] and [25]. We then showed that applying polarizing transforms to multiple processes separately not only polarizes the processes, but the correlations are also 
polarized. We saw that coding schemes exploiting this joint polarization phenomenon achieve some, but not always all, optimal points in the rate regions of the mentioned problems, with error probabilities comparable to those of single-user polar coding schemes. The results in this section are from [41] and [1]. One should note that the unachievability of certain rate points by this scheme is not due to the way that the processes are polarized - they are indeed polarized using the same transform as in the first method discussed above - but rather to the proposed decoding order, which does not fully exploit the resulting probability structure. This rate loss is a good example that illustrates the strong dependence of polarization on how the probability structure in a process is decomposed through the choice of the decoding algorithm. Indeed, Arıkan recently showed that any optimal rate point in two-user settings can be achieved if the decoding order is chosen suitably [8].

The focus of this monograph was on the fundamentals of polarization theory, we thus had to neglect several interesting early results in the field. At the time of this writing, polar coding research encompassed more than one hundred publications. We will only mention a few of these results for reference. Our hope is that the material covered here will familiarize the researcher with the techniques and thus make related work more accessible.

Some of the early work was on applying polar coding ideas to various communication scenarios. In [28], it was shown that polar coding achieves the rate-distortion bound for a symmetric binary source under Hamming distortion. Later, this result was generalized to arbitrary sources with prime reconstruction alphabets [24]. Korada showed in [25] that polar codes also achieve optimal rates in certain instances of the Gelfand-Pinsker problem, the Wyner-Ziv problem, and the degraded broadcast channel problem. Polar coding for degraded relay channels was first studied in [3] where the source-to-destination and the relayto-destination channels were assumed orthogonal. Later in [23] it was shown that polar codes achieve the capacity of general binary-input symmetric degraded relay channels. Polar codes for special cases of broadcast channels were discussed in [25] and [15]. Polar coding for wiretap channels was studied concurrently by several groups. Secrecy 
capacity-achieving properties of polar codes for degraded symmetric wiretap channels were established independently in $[3,21,29,32]$. Roughly, all of these results are obtained by showing that the random coding arguments used in the canonical achievability proofs can be mimicked by polar coding. Polar coding for multiple access channels (Section 6) was extended in [2] to channels with more than two senders.

As we mentioned above, the original error probability analysis in [9] (Section 2) is asymptotic, and we saw that polarization does not take place sufficiently fast to make polar codes useful at short blocklengths. There has been considerable effort in refining the error probability analysis of these codes, as well as in improving their performance. In [46] and [20], the asymptotic analysis of [9] was refined to give ratedependent bounds on the error probability. This refinement invokes the central limit theorem in addition to the law of large numbers used in the original analysis. Another way to study the error probabilityrate tradeoff is to determine the achievable rates (as a function of the blocklength) given a target error probability. This 'scaling' study was undertaken in [27] and [16]. As we saw in Section 2.5, performance improvements were reported in [44] and [7]. Hussami et al. [22] also reported gains in error probability under belief propagation decoding. Clearly, all of these are encouraging developments in polar coding research, and thus an understanding of the reasons for these gains is needed.

Another practical consideration in channel coding is robustness against uncertainty in the channel. This is often studied as a compound channel problem, where the task is to design a code that will perform well over all memoryless channels in a given class. Polar coding for compound channels was considered in [18] by Hassani et al., where it was shown that over a compound channel that includes the binary symmetric and binary erasure channels with equal capacities, polar codes achieve strictly smaller rates than the compound channel capacity under SC decoding. In [39, pp. 87-89], it is shown that this gap to capacity is indeed due to the suboptimality of the SC decoder, and can be closed by employing optimal decoders at the receiver. An open problem of interest is to determine whether polar 
codes achieve compound channel capacity under low-complexity decoding algorithms. Uncertainty can also be present in the form of channel memory. A preliminary result to this end was given in [38], where it was shown that Arıkan's construction polarizes a large class of processes with memory.

Although it has been amply demonstrated that polarization is a fairly general phenomenon, the extent of the practical and the theoretical implications of this generality remains largely unknown. 


\section{Acknowledgments}

I am grateful to Professors Erdal Arıkan, Emre Telatar, Rüdiger Urbanke, and Alexander Vardy for their feedback on an earlier version of this monograph. Two anonymous reviewers provided very useful comments that helped improved the exposition. I would also like to thank Professor Sergio Verdú for inviting me to write for the Foundations and Trends series. 


\section{References}

[1] E. Abbe, "Randomness and dependencies extraction via polarization," in Proceedings of the Information Theory and Applications Workshop, Feb. 2011.

[2] E. Abbe and E. Telatar, "Polar codes for the m-user MAC," [Online] Available: arXiv:1002.0777, August 2010.

[3] M. Andersson, V. Rathi, R. Thobaben, J. Kliewer, and M. Skoglund, "Nested polar codes for wiretap and relay channels," IEEE Communications Letters, vol. 14, no. 8, pp. 752-754, August 2010.

[4] E. Arıkan, "Channel polarization: A method for constructing capacityachieving codes for symmetric binary-input memoryless channels," IEEE Transactions on Information Theory, vol. 55, no. 7, pp. 3051-3073, July 2009.

[5] E. Arıkan, "Source polarization," in Proceedings of the International Symposium on Information Theory, pp. 899-903, 13-18 June 2010.

[6] E. Arıkan, "A survey of Reed-Muller codes from polar coding perspective," in Proceedings of the Information Theory Workshop, 1-5, Jan. 2010.

[7] E. Arıkan, "Systematic polar coding," IEEE Communications Letters, vol. 15, no. 8, pp. 860-862, August 2011.

[8] E. Arrkan, "Polar coding for the Slepian-Wolf problem based on monotone chain rules," in Proceedings of the International Symposium on Information Theory, pp. 566-570, July 2012.

[9] E. Arıkan and E. Telatar, "On the rate of channel polarization," in Proceedings of the International Symposium on Information Theory, pp. 1493-1495.

[10] J. A. Bondy and U. S. R. Murty, Graph Theory. New York: Springer, 2008.

[11] A. Clark, Elements of Abstract Algebra. New York: Dover, 1971.

[12] D. J. Costello Jr. and G. D. Forney Jr., "Channel coding: The road to channel capacity," Proceedings of the IEEE, vol. 95, no. 6, June 2007. 
[13] I. Csiszár and J. Körner, Information Theory: Coding Theorems for Discrete Memoryless Systems. New York: Academic Press, 1981.

[14] R. G. Gallager, Information Theory and Reliable Communication. New York: Wiley, 1968.

[15] N. Goela, E. Abbe, and M. Gastpar, "Polar codes for the deterministic broadcast channel," in Proceedings of the International Zurich Seminars on Communications, pp. 51-54, Feb-Mar 2012.

[16] A. Goli, H. Hassani, and R. Urbanke, "Universal bounds on the scaling behavior of polar codes," in Proceedings of the International Symposium on Information Theory, July 2012.

[17] A. Grant, B. Rimoldi, R. Urbanke, and P. Whiting, "Rate-splitting multiple access for discrete memoryless channel," IEEE Transactions on Information Theory, vol. 47, no. 3, pp. 873-890, March 2001.

[18] H. Hassani, S. B. Korada, and R. Urbanke, "The compound capacity of polar codes," in Proceedings of the Annual Allerton Conference on Communications, Control, and Computing, pp. 16-21, Sept-Oct 2009.

[19] H. Hassani, R. Mori, T. Tanaka, and R. Urbanke, "Rate-dependent analysis of the asymptotic behavior of channel polarization," submitted to IEEE Transactions on Information Theory, [Online] Available: arXiv:1110.0194, Oct 2011.

[20] H. Hassani and R. Urbanke, "On the scaling of polar codes: I. The behavior of polarized channels," in Proceedings of the International Symposium on Information Theory, pp. 874-878, June 2010.

[21] E. Hof and S. Shamai, "Secrecy-achieving polar coding," in Proceedings of the Information Theory Workshop, pp. 1-5, August 2010.

[22] N. Hussami, S. B. Korada, and R. Urbanke, "Performance of polar codes for channel and source coding," in Proceedings of the International Symposium on Information Theory, pp. 1488-1492, July 2009.

[23] M. Karzand, "Polar codes for degraded relay channels," in Proceedings of the International Zurich Seminar on Communications, pp. 59-62, Feb-Mar 2012.

[24] M. Karzand and E. Telatar, "Polar codes for q-ary source coding," in Proceedings of the International Symposium on Information Theory, pp. 909-912, June 2010 .

[25] S. B. Korada, "Polar codes for channel and source coding," PhD Dissertation, EPFL 2009.

[26] S. B. Korada, E. Şaşoğlu, and R. Urbanke, "Polar codes: Characterization of exponent, bounds, and constructions," IEEE Transactions on Information Theory, vol. 56, no. 12, pp. 6253-6264, Dec 2010.

[27] S. B. Korada, A. Montanari, E. Telatar, and R. Urbanke, "An empirical scaling law for polar codes," in Proceedings of the International Symposium on Information Theory, pp. 884-888, June 2010.

[28] S. B. Korada and R. Urbanke, "Polar codes are optimal for lossy source coding," IEEE Transactions on Information Theory, vol. 56, no. 4, pp. 1751-1768, April 2010.

[29] O. O. Koyluoglu and H. El Gamal, "Polar coding for secure transmission and key agreement," International Symposium on Pers. Ind. Mob. Radio Comm., pp. 2698-2703, Sep 2010. 
[30] C. Leroux, I. Tal, A. Vardy, and W. J. Gross, "Hardware architectures for successive cancellation decoding of polar codes," International Conference on Acou., Sp., and Sig. Proc., pp. 1665-1668, May 2011.

[31] F. J. MacWilliams and N. J. Sloane, The Theory of Error-Correcting Codes. North-Holland, 1977.

[32] H. Mahdavifar and A. Vardy, "Achieving the secrecy capacity of wiretap channels using polar codes," IEEE Transactions on Information Theory, vol. 57, no. 10, pp. 6428-6443, Oct 2011.

[33] R. Mori and T. Tanaka, "Performance and construction of polar codes on symmetric binary-input memoryless channels," in Proceedings of the International Symposium on Information Theory, pp. 1496-1500, July 2009.

[34] R. Mori and T. Tanaka, "Channel polarization on q-ary discrete memoryless channels by arbitrary kernels," in Proceedings of the International Symposium on Information Theory, pp. 894-898, June 2010.

[35] W. Park and A. Barg, "Multilevel polarization for nonbinary codes and parallel channels," in Proceedings of the Annual Allerton Conference on Communications, Control, and Computing, pp. 228-234, Sep 2011.

[36] R. Pedarsani, S. H. Hassani, I. Tal, and E. Telatar, "On the construction of polar codes," in Proceedings of the International Symposium on Information Theory, pp. 11-15, Aug 2011.

[37] E. Şaşoğlu, "An entropy inequality for q-ary random variables and its application to channel polarization," in Proceedings of the International Symposium on Information Theory, pp. 1360-1363, June 2010.

[38] E. Şaşoğlu, "Polarization in the presence of memory," in Proceedings of the International Symposium on Information Theory, pp. 189-193, June 2010.

[39] E. Şaşoğlu, "Polar coding theorems for discrete systems," PhD Dissertation, EPFL 2011.

[40] E. Şaşoğlu, E. Telatar, and E. Arıkan, "Polarization for arbitrary discrete memoryless channels," in Proceedings of the Information Theory Workshop, pp. 144148, Oct 2009.

[41] E. Şaşoğlu, E. Telatar, and E. Yeh, "Polar codes for the two-user multiple-access channel," [Online]. Available: arXiv:1006.4255, June 2010.

[42] C. E. Shannon, "A mathematical theory of communication," Bell System Technology Journal, vol. 27, pp. 379-423, 623-565, July and October 1948.

[43] D. Slepian and J. Wolf, "Noiseless coding of correlated information sources," IEEE Transactions on Information Theory, vol. 19, no. 4, pp. 471-480, 1973.

[44] I. Tal and A. Vardy, "List decoding of polar codes," in International Symposium on Information Theory, pp. 1-5, Aug 2011.

[45] I. Tal and A. Vardy, "How to construct polar codes," [Online]. Available: arXiv:1105.6164, May 2011.

[46] T. Tanaka and R. Mori, "Refined rate of channel polarization," in International Symposium on Information Theory, pp. 889-893, June 2010.

[47] E. Telatar, private communication.

[48] A. D. Wyner and J. Ziv, "A theorem on the entropy of certain binary sequences and applications: Part I," IEEE Transactions on Information Theory, vol. 19, no. 6, pp. 769-772, Nov 1973. 